\title{
English translations of twenty-one of Ertel's papers on geophysical fluid dynamics
}

\author{
WAyne Schubert ${ }^{1 *}$, Eberhard Ruprecht ${ }^{2}$, Rolf Hertenstein $^{1}$, ROSANA Nieto Ferreira $^{3}$,

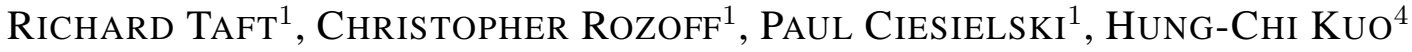 \\ ${ }^{1}$ Department of Atmospheric Science, Colorado State University, USA \\ ${ }^{2}$ Leibniz-Institut für Meereswissenschaften an der Universität Kiel \\ ${ }^{3}$ GEST Center, University of Maryland, USA \\ ${ }^{4}$ Department of Atmospheric Sciences, National Taiwan University, Taiwan
}

(Manuscript submitted January 20, 2004)

\begin{abstract}
This paper contains a collection of English translations of twenty-one of Hans Ertel's papers on geophysical fluid dynamics. The selected papers were originally published between 1942 and 1970 in either German or Spanish. This collection includes the four classic 1942 papers on vorticity and potential vorticity conservation principles and also papers on generalized conservation relations, hydrodynamic commutation formulas, Clebsch and Weber transformations, and isogons and isotachs in two-dimensional flows.

\section{Zusammenfassung}

Dieser Artikel ist eine Sammlung von 21 Veröffentlichungen zur Strömungsdynamik von Hans Ertel. Die ausgewählten Artikel erschienen ursprünglich in deutscher oder spanischer Sprache im Zeitraum von 1942 bis 1970. In der Sammlung enthalten sind die vier klassischen Artikel von 1942 über Vorticity und die Erhaltung potentieller Vorticity, sowie weitere Artikel zu allgemeinen Erhaltungssätzen, hydrodynamischen Vertauschungsrelationen, Clebsch- und Weber-Transformationen, und Isogonen und Isotachen in zweidimensiononalen Strömungen.
\end{abstract}

\section{Preface}

In view of the central role played by Ertel's potential vorticity in modern geophysical fluid dynamics, it may seem surprising that few of our fellow scientists have read Ertel's original works on the subject. This may be because most of these original papers were not written in English and were published in journals not easily accessible to many of us. It is in hopes of making Ertel's works more accessible that we provide the following set of translations.

The complete bibliography of Ertel's scientific contributions, published between 1929 and 1972, lists a staggering 272 papers. Most of the papers are short, containing a concise mathematical argument and a brief physical interpretation (figures are rare). The majority of the papers are on meteorology, but there also appear papers on oceanography, cosmology, atomic physics, volcanoes, fluvial erosion, etc. In selecting the papers for this special issue we have limited the topic to geophysical fuid dynamics and have been greatly aided by the collection of original German and Spanish articles reproduced in the five "Ertel volumes" edited by
W. Schröder and H.-J. Treder, published in cooperation with the Interdivisional Commission on History of the International Association of Geomagnetism and Aeronomy (IAGA). The twenty-one translations presented here are as follows:

1. ERTEL, H., 1942a: Ein neuer hydrodynamischer Wirbelsatz. Meteorol. Z. (Braunschweig), 59(9), S. 277-281.

2. ERTEL, H., 1942b: Ein neuer hydrodynamischer Erhaltungssatz. Die Naturwiss. (Berlin), 30(36), S. 543-544.

3. ERTEL, H., 1942c: Über hydrodynamische Wirbelsätze. Physik. Z. (Leipzig), 43, S. 526-529.

4. ERTel, H., 1942d: Über das Verhältnis des neuen hydrodynamischen Wirbelsatzes zum Zirkulationssatz von V. Bjerknes. Meteorol. Z. (Braunschweig), 59(12), S. 385-387.

5. ERTEL, H., 1952: Über die physikalische Bedeutung von Funktionen, welche in der Clebsch-Transformation der hydrodynamischen Gleichungen auftreten. Sitz.-Ber. Dt. Ak. d. Wiss.

${ }^{*}$ Corresponding author: Wayne Schubert, Department of Atmospheric Science, Colorado State University, Fort Collins, Colorado, USA, 80523, email: waynes@atmos.colostate.edu 
Berlin, Klasse f. Math. u. allg. Naturwiss., 1952, No. 3, (Berlin 1952), S. 3-18.

6. ERTEL, H., 1955a: Kanonischer Algorithmus hydrodynamischer Wirbelgleichungen. Sitz.-Ber. Dt. Ak. d. Wiss. Berlin, Klasse f. Math. u. allg. Naturwiss., 1954, No. 4, (Berlin 1955), S. 3-10.

7. ERTEL, H., 1955b: Ein neues Wirbel-Theorem der Hydrodynamik. Sitz.-Ber. Dt. Ak. d. Wiss. Berlin, Klasse f. Math. u. allg. Naturwiss., 1954, No. 5, (Berlin 1955), S. 5-11.

8. ERTEL, H., 1956: Orthogonale Trajektoriensysteme in stationären ebenen Strömungsfeldern inkompressibler idealer Flüssigkeiten. Dt. Ak. d. Wissenschaften zu Berlin 1946-1956; Aus der Klasse für Mathematik, Physik und Technik, Berlin, S. 67-71.

9. ERTEL, H., 1957: Sobre una relación general entre la velocidad del viento y la intensidad del campo hidrodinámico en la atmósfera. Gerlands Beitr. z. Geophysik (Leipzig), 66(4), p. 323-329.

10. ERTEL, H., 1960a: Teorema sobre invariantes sustanciales de la Hidrodinámica. Gerlands Beitr. z. Geophysik (Leipzig), 69(5), p. 290-293.

11. ERTEL, H., 1960b: Relación entre la derivada individual y una cierta divergencia espacial en Hidrodinámica. Gerlands Beitr. z. Geophysik (Leipzig), 69(6), p. 357-361.

12. ERTEL, H., 1961: Isoclinas e isótacas en corrientes potenciales bidimensionales de un flúido incompresible. Gerlands Beitr. z. Geophysik (Leipzig), 70(1), p. 55-58.

13. ERTEL, H., 1963a: Analogía entre las ecuaciones del movimiento y las ecuaciones del torbellino en la Hidrodinámica. Gerlands Beitr. z. Geophysik (Leipzig), 72(5), p. 312-314.

14. ERTEL, H., 1963b: Relaciones entre operaciones diferenciales del cálculo vectorial y paréntesis de Lagrange, con aplicación a la Hydrodinámica. Geofi sica pura e appl. (Milano), 55, 1963/II, p. 119-122.

15. ERTEL, H., 1964: El número máximo de invariantes independientes en Hidrodinámica. Revista de Geofísica (Madrid), XXIII, Nums. 91/92, p. 121-124.

16. ERTEL, H., 1965a: Hydrodynamische Vertauschungs-Relationen. Acta Hydrophysica, IX(3), Berlin, S. 115-123.

17. ERTEL, H., 1965b: Kommutative Operatoren instationärer Strömungsfelder perfekter, piezotroper Flüssigkeiten. Monatsber. Dt. Ak. d. Wiss. Berlin, 7(4), S. 296-298.
18. ERTEL, H., 1965c: Theorem über die unimodulare Transformation hydrodynamischer Numerierungskoordinaten. Gerlands Beitr. z. Geophysik (Leipzig), 74(3), S. 255-260.

19. ERTEL, H., 1970a: Eine Relation zwischen kinematischen Parametern horizontaler Strömungsfelder in der Atmosphäre. Időjárás Budapest, 74(1-2), S. 98-102.

20. ERTEL, H., 1970b: Spin und Deformationstensor im Zusammenhand mit Isogonen und Isotachen in ebenen Strömmungsfeldern. Acta Geodaet., Geophys. et Montanist. Acad. Sci. Hung., Tomus 5 (3-4), Budapest, pp. 383-387.

21. ERTEL, H., 1970c: Transformation der Differentialform der Weberschen hydrodynamischen Gleichungen unter Berücksichtigung der Erdrotation. Gerlands Beitr. z. Geophysik (Leipzig), 79(6), p. $421-424$.

Some of these papers are best studied in groups. For example, papers 1-4 present the results on potential vorticity with which we are so familiar. When these four papers were published in 1942, many readers probably thought that the new potential vorticity equation was very elegant, but with practical applications limited to tracer studies using special isentropic analyses. Later developments, however, would prove that Ertel's potential vorticity is much more useful than this simple initial understanding. The potential vorticity $\rho^{-1}(2 \Omega+\nabla \times \mathbf{v}) \cdot \nabla \theta$ is a combination of the wind and mass fields. For general nonbalanced fbws, knowledge of the potential vorticity field is not sufficient to determine both wind and mass. However, largescale atmospheric fbws are quasi-static, and the wind and mass fields have mutually adjusted so that there exists some kind of approximate balance between them (e.g., geostrophic, gradient, or nonlinear balance). In such situations, the density $\rho$, the velocity $\mathbf{v}$, and the potential temperature $\theta$ can all be expressed in terms of the pressure $p$, so that the potential vorticity determines everything, if you can just invert it to find $p$. Thus, it is the concept of balance coupled with potential vorticity conservation which proves to be such a powerful tool in analyzing geophysical fbws. In addition, the transformation to different horizontal coordinates (e.g., geostrophic coordinates) or a different vertical coordinate (potential temperature) can often simplify the mathematics and lead to concise and elegant descriptions of the dynamics. In any case, the fundamental underlying notion is Ertel's potential vorticity (or an appropriate approximation to it); this is the motivation for our continuing interest in these classic 1942 papers. 
Papers 6, 10, 11, and

22. Ertel, H., and C.-G. Rossby, 1949: A new conservation theorem of hydrodynamics. Geofi sica Pura e Appl. (Milano), 14, Fasc. 3-4, pp. 189-193.

can also be read as a group. The latter paper (22) is not included in the present collection since it was published in English (one of only two of Ertel's papers that were published in English). This group of papers deals with generalizations of the original 1942 results. In particular paper 6 provides a canonical algorithm from which the results of both the group 1-4 and paper 22 are easily obtained.

Finally, papers $8,12,19$, and 20 can also be read as a group. Reffecting Ertel's interest in connecting dynamic meteorology with operational practice in synoptic meteorology, they deal with isogons and isotachs in two-dimensional fbw and the use of natural coordinate systems.

In producing these translations we have attempted to preserve the formatting of the original papers as much as possible. References, for example, are cited in each paper with a style that matches that paper's original formatting, and the reference lists at the end of each paper are presented in their original untranslated forms. Certain symbols and notations, however, were updated to more modern conventions. For example, vector quantities have been represented using boldface symbols (rather than using an arrow symbol), and the " $\nabla$ " symbol was used in place of the "grad" operator. Also, some formatting modifications were made in order to develop some coherency and consistency across the collection of papers as a whole (such as the formatting of section headings and equation numbers) and to deal with the two-column format of this journal (this affected the formatting of many of the longer formulas). Although Ertel's papers are largely free of typographical errors in the equations, we did detect a few obvious ones, and these have been corrected in the translations.

\section{ERTEL (1942a): A new hydrodynamical vorticity equation}

Relative to a rotating coordinate system, whose rotation is given by the constant rotation vector $\Omega$, one can write the Eulerian hydrodynamical equation for an ideal compressible flid,

$$
\begin{aligned}
\frac{\partial \mathbf{v}}{\partial t}+\nabla\left(\frac{1}{2} \mathbf{v}^{2}\right) & -\mathbf{v} \times(\nabla \times \mathbf{v})+2 \boldsymbol{\Omega} \times \mathbf{v} \\
& =-\nabla \Phi-\alpha \nabla p
\end{aligned}
$$

together with the continuity equation

$$
\frac{d \rho}{d t}=\frac{\partial \rho}{\partial t}+\mathbf{v} \cdot \nabla \rho=-\rho \nabla \cdot \mathbf{v} .
$$

The foregoing symbols have the usual meaning $(\alpha=$ $1 / \rho$ is the specific volume, $\Phi$ the potential of external forces).

The velocity $\mathbf{v}_{e}$ is related to the position vector $\mathbf{r}$ through

$$
\mathbf{v}_{e}=\boldsymbol{\Omega} \times \mathbf{r} .
$$

The velocity of a mass element relative to an inertial system (non-rotating system) then becomes

$$
\mathbf{v}+\mathbf{v}_{e}=\mathbf{v}+\mathbf{\Omega} \times \mathbf{r} .
$$

Since

$$
\nabla \times(\boldsymbol{\Omega} \times \mathbf{r})=2 \boldsymbol{\Omega}
$$

then

$$
\mathbf{Z}=\nabla \times\left(\mathbf{v}+\mathbf{v}_{e}\right)=\nabla \times \mathbf{v}+2 \boldsymbol{\Omega},
$$

is the absolute vorticity, i.e., the vorticity relative to an inertial system, so that the Eulerian equation can be written

$$
\frac{\partial \mathbf{v}}{\partial t}+\nabla\left(\frac{1}{2} \mathbf{v}^{2}\right)-\mathbf{v} \times \mathbf{Z}=-\nabla \Phi-\alpha \nabla p .
$$

Using

$$
\nabla \times(\alpha \nabla p)=-\nabla p \times \nabla \alpha
$$

and taking the curl of (1.7), we obtain

$$
\frac{\partial}{\partial t} \nabla \times \mathbf{v}-\nabla \times(\mathbf{v} \times \mathbf{Z})=\nabla p \times \nabla \alpha .
$$

Now consider $\psi=\psi(\mathbf{r}, t)$ to be a hydrodynamical invariant, i.e. a physical property which is conserved for every fluid element:

$$
\frac{d \psi}{d t}=\frac{\partial \psi}{\partial t}+\mathbf{v} \cdot \nabla \psi=0
$$

as for example the potential temperature under adiabatic processes, or the polytropic temperature under polytropic conditions ${ }^{1}$, or the total water content under conditions of no precipitation and without moisture input. In general, the invariant $\psi$ does not have the same 
value for all particles, but gives a field of $\psi=$ constant surfaces ${ }^{2}$.

Scalar multiplication of (1.9) by $\nabla \psi$ yields

$$
\begin{aligned}
\nabla \psi \cdot \frac{\partial}{\partial t} \nabla \times \mathbf{v} & -\nabla \psi \cdot \nabla \times(\mathbf{v} \times \mathbf{Z}) \\
& =(\nabla p \times \nabla \alpha) \cdot \nabla \psi
\end{aligned}
$$

or

$$
\nabla \psi \cdot \frac{\partial \mathbf{Z}}{\partial t}-\nabla \psi \cdot \nabla \times(\mathbf{v} \times \mathbf{Z})=(\nabla p \times \nabla \alpha) \cdot \nabla \psi,
$$

since from (1.6)

$$
\frac{\partial \mathbf{Z}}{\partial t}=\frac{\partial}{\partial t} \nabla \times \mathbf{v}
$$

Now, using

$$
\nabla \cdot[\nabla \psi \times(\mathbf{v} \times \mathbf{Z})]=-\nabla \psi \cdot \nabla \times(\mathbf{v} \times \mathbf{Z}),
$$

\section{(1.12) becomes}

$$
\nabla \psi \cdot \frac{\partial \mathbf{Z}}{\partial t}+\nabla \cdot[\nabla \psi \times(\mathbf{v} \times \mathbf{Z})]=(\nabla p \times \nabla \alpha) \cdot \nabla \psi .
$$

The vector algebra formula (triple vector product formula)

$$
\mathbf{a} \times(\mathbf{b} \times \mathbf{c})=\mathbf{b}(\mathbf{c} \cdot \mathbf{a})-\mathbf{c}(\mathbf{b} \cdot \mathbf{a})
$$

results in

$$
\nabla \psi \times(\mathbf{v} \times \mathbf{Z})=\mathbf{v}(\mathbf{Z} \cdot \nabla \psi)-\mathbf{Z}(\mathbf{v} \cdot \nabla \psi),
$$

which, along with

$$
\mathbf{v} \cdot \nabla \psi=-\frac{\partial \psi}{\partial t}
$$

allows us to write

$$
\nabla \psi \times(\mathbf{v} \times \mathbf{Z})=\mathbf{v}(\mathbf{Z} \cdot \nabla \psi)+\mathbf{Z} \frac{\partial \psi}{\partial t} .
$$

Taking the divergence, we obtain

$$
\begin{aligned}
\nabla \cdot[\nabla \psi & \times(\mathbf{v} \times \mathbf{Z})]=\mathbf{v} \cdot \nabla(\mathbf{Z} \cdot \nabla \psi) \\
& +(\mathbf{Z} \cdot \nabla \psi) \nabla \cdot \mathbf{v}+\mathbf{Z} \cdot \frac{\partial}{\partial t} \nabla \psi
\end{aligned}
$$

since (1.5) and (1.6) imply

$$
\nabla \cdot \mathbf{Z}=0 .
$$

Substitution of (1.19) into (1.15) results in

$$
\begin{aligned}
& \nabla \psi \cdot \frac{\partial \mathbf{Z}}{\partial t}+\mathbf{v} \cdot \nabla(\mathbf{Z} \cdot \nabla \psi)+\mathbf{Z} \cdot \frac{\partial}{\partial t} \nabla \psi \\
& \quad+(\mathbf{Z} \cdot \nabla \psi) \nabla \cdot \mathbf{v}=(\nabla p \times \nabla \alpha) \cdot \nabla \psi
\end{aligned}
$$

which, because of

$$
\nabla \psi \cdot \frac{\partial \mathbf{Z}}{\partial t}+\mathbf{Z} \cdot \frac{\partial}{\partial t} \nabla \psi=\frac{\partial}{\partial t}(\mathbf{Z} \cdot \nabla \psi)
$$

and

$$
\frac{\partial}{\partial t}(\mathbf{Z} \cdot \nabla \psi)+\mathbf{v} \cdot \nabla(\mathbf{Z} \cdot \nabla \psi)=\frac{d}{d t}(\mathbf{Z} \cdot \nabla \psi),
$$

gives

$$
\frac{d}{d t}(\mathbf{Z} \cdot \nabla \psi)+(\mathbf{Z} \cdot \nabla \psi) \nabla \cdot \mathbf{v}=(\nabla p \times \nabla \alpha) \cdot \nabla \psi .
$$

Division by $\rho=1 / \alpha$ and use of the equation

$$
\frac{1}{\rho} \nabla \cdot \mathbf{v}=-\frac{1}{\rho^{2}} \frac{d \rho}{d t}=\frac{d}{d t}\left(\frac{1}{\rho}\right)=\frac{d \alpha}{d t}
$$

results in

$$
\frac{d}{d t}(\alpha \mathbf{Z} \cdot \nabla \psi)=\alpha(\nabla p \times \nabla \alpha) \cdot \nabla \psi,
$$

an equation whose right hand side has a very simple physical meaning. To see this, consider the form

$$
\left(\nabla \alpha^{\prime} \times \nabla \beta^{\prime}\right) \cdot \nabla \gamma^{\prime}
$$

In so far as there is no homogeneous relation $F\left(\alpha^{\prime}, \beta^{\prime}\right)=0$ between the scalar functions $\alpha^{\prime}$ and $\beta^{\prime}$, the surfaces $\alpha^{\prime}(\mathbf{r}, t)=$ constant and $\beta^{\prime}(\mathbf{r}, t)=$ constant form a solenoid system. If the third scalar function $\gamma^{\prime}$ is not of the form $\gamma^{\prime}=\gamma^{\prime}\left(\alpha^{\prime}, \beta^{\prime}\right)$, then this solenoid is divided into cells between the surfaces $\gamma^{\prime}(\mathbf{r}, t)=$ constant. Assume all three families of surfaces are given by

$$
\begin{aligned}
& \ldots, \alpha^{\prime}-1, \alpha^{\prime}, \alpha^{\prime}+1, \ldots \\
& \ldots, \beta^{\prime}-1, \beta^{\prime}, \beta^{\prime}+1, \ldots \\
& \ldots, \gamma^{\prime}-1, \gamma^{\prime}, \gamma^{\prime}+1, \ldots
\end{aligned}
$$

so that we have unit cells. Such a unit cell is shown in Fig. 1.1. It is formed by the intersections of the surfaces

$$
\begin{aligned}
& \alpha^{\prime}=\text { constant } \quad \text { and } \quad \alpha^{\prime}+1=\text { constant } \\
& \beta^{\prime}=\text { constant } \quad \text { and } \quad \beta^{\prime}+1=\text { constant } \\
& \gamma^{\prime}=\text { constant } \quad \text { and } \quad \gamma^{\prime}+1=\text { constant }
\end{aligned}
$$

The surface $F$ in Fig. 1.1 is perpendicular to $\nabla \alpha^{\prime} \times$ $\nabla \beta^{\prime}$ and it contains the directions of the gradient of $\alpha^{\prime}$ (which is the direction a) and of the gradient of $\beta^{\prime}$ (which is the direction $\mathbf{b}$ ). The direction of $\mathbf{c}$ is that of the gradient of $\gamma^{\prime}$ which is perpendicular to the surface of constant $\gamma^{\prime}$. The vector $\mathbf{c}$ and the normal of $\mathrm{F}$ form the angle $\nu$. The vectors $\mathbf{a}$ and $\mathbf{b}$ lie in the surface $\mathrm{F}$ and 


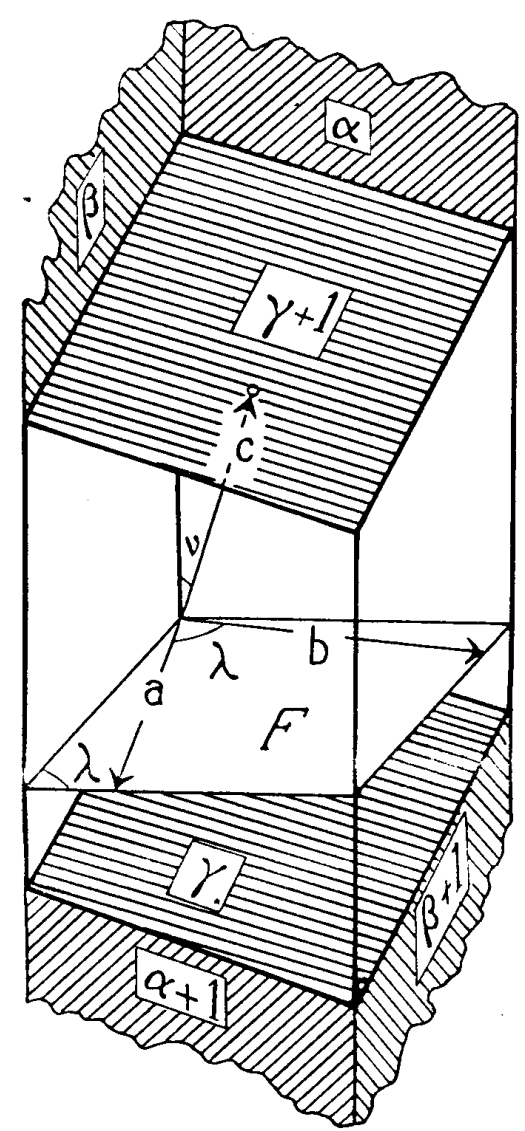

Fig. 1.1

form the angle $\lambda$. This is also (except for higher order terms) the angle between the surface $\alpha^{\prime}+1$ and the surface $\beta^{\prime}$ (and between the surface $\alpha^{\prime}$ and the surface $\beta^{\prime}+$ 1), because $\mathbf{a} \perp \alpha^{\prime}\left(\mathbf{a} \perp \alpha^{\prime}+1\right)$ and $\mathbf{b} \perp \beta^{\prime}\left(\mathbf{b} \perp \beta^{\prime}+\right.$ 1). The length of $\mathbf{c}$ is the height of the unit cell, because c gives the distance between the surfaces of $\gamma^{\prime}+1$ and $\gamma^{\prime}$. The surface area of $\mathrm{F}$ is

$$
F=\frac{a b}{\sin \lambda}
$$

because $a / \sin \lambda$ is the side of $\mathbf{F}$ to which $\mathbf{b}$ is perpendicular. Because the perpendicular to $\gamma^{\prime}$ and the perpendicular to $\mathrm{F}$ form the angle $\nu$, the projection of $\mathrm{F}$ onto $\gamma^{\prime}$ is given by

$$
\text { projection of } F=\frac{F}{\cos \nu} .
$$

The volume $V$ of the unit cell is $c(F / \cos \nu)$, or

$$
V=\frac{a b c}{\sin \lambda \cos \nu} \text {. }
$$

Now,

$$
\begin{aligned}
\left(\nabla \alpha^{\prime}\right. & \left.\times \nabla \beta^{\prime}\right) \cdot \nabla \gamma^{\prime} \\
& =\left|\nabla \alpha^{\prime}\right|\left|\nabla \beta^{\prime}\right| \sin \lambda\left|\nabla \gamma^{\prime}\right| \cos \nu
\end{aligned}
$$

and since

$$
\left.\begin{array}{l}
\left|\nabla \alpha^{\prime}\right|=\frac{\left(\alpha^{\prime}+1\right)-\alpha^{\prime}}{a}=\frac{1}{a} \\
\left|\nabla \beta^{\prime}\right|=\frac{\left(\beta^{\prime}+1\right)-\beta^{\prime}}{b}=\frac{1}{b} \\
\left|\nabla \gamma^{\prime}\right|=\frac{\left(\gamma^{\prime}+1\right)-\gamma^{\prime}}{c}=\frac{1}{c}
\end{array}\right\},
$$

we have

$$
\left(\nabla \alpha^{\prime} \times \nabla \beta^{\prime}\right) \cdot \nabla \gamma^{\prime}=\frac{\sin \lambda \cos \nu}{a b c},
$$

which together with (1.29) gives

$$
\left(\nabla \alpha^{\prime} \times \nabla \beta^{\prime}\right) \cdot \nabla \gamma^{\prime}=\frac{1}{V},
$$

which is the inverse volume of an $\left(\alpha^{\prime}, \beta^{\prime}, \gamma^{\prime}\right)$ unit cell or the number of $\left(\alpha^{\prime}, \beta^{\prime}, \gamma^{\prime}\right)$ unit cells in a unit volume. Thus,

$$
\alpha\left(\nabla \alpha^{\prime} \times \nabla \beta^{\prime}\right) \cdot \nabla \gamma^{\prime}=\frac{\alpha}{V}=N\left(\alpha^{\prime}, \beta^{\prime}, \gamma^{\prime}\right),
$$

the number of $\left(\alpha^{\prime}, \beta^{\prime}, \gamma^{\prime}\right)$ unit cells in the specific volume, i.e. in the volume occupied by a unit mass of fuid. Then,

$$
\alpha(\nabla p \times \nabla \alpha) \cdot \nabla \psi=N(p, \alpha, \psi)
$$


the number of $(p, \alpha, \psi)$ unit cells in the specific volume or the number of $(p, \alpha, \psi)$ unit cells per unit mass. The sign of $N(p, \alpha, \psi)$ is positive if the vectors $\nabla p, \nabla \alpha$ and $\nabla \psi$ are cyclic; otherwise it is negative. Thus, we have

$$
\begin{aligned}
(\nabla p \times \nabla \alpha) \cdot \nabla \psi & =(\nabla \psi \times \nabla p) \cdot \nabla \alpha \\
& =(\nabla \alpha \times \nabla \psi) \cdot \nabla p
\end{aligned}
$$

or

$$
N(p, \alpha, \psi)=N(\psi, p, \alpha)=N(\alpha, \psi, p),
$$

that is, the sign of $N(p, \alpha, \psi)$ remains unchanged during cyclic changes. Because of

$$
\mathbf{a} \times \mathbf{b}=-\mathbf{b} \times \mathbf{a},
$$

during non-cyclic changes, we have

$$
N(p, \alpha, \psi)=-N(p, \psi, \alpha)=-N(\psi, \alpha, p) .
$$

Equation (1.26) can now be written as

$$
\begin{aligned}
\frac{d}{d t}(\alpha \mathbf{Z} \cdot \nabla \psi) & =\frac{d}{d t}\{\alpha(\nabla \times \mathbf{v}+2 \boldsymbol{\Omega}) \cdot \nabla \psi\} \\
& =N(p, \alpha, \psi)
\end{aligned}
$$

which is the analytical form of the following vorticity theorem:

The individual time change of the specific volume multiplied by the scalar product of the absolute vorticity vector and the gradient of a conservative property of every fluid particle is equal to the number of $(p, \alpha, \psi)$ unit cells contained in the specific volume $\alpha$.

An important special case of the foregoing new vorticity theorem occurs when the conservative property $\psi$ can be written as a function of $p$ and $\alpha$,

$$
\psi=\psi(p, \alpha),
$$

for example, in the case when $\psi$ is the polytropic temperature (see R. Emden). Then we have

$$
\nabla \psi=\frac{\partial \psi}{\partial p} \nabla p+\frac{\partial \psi}{\partial \alpha} \nabla \alpha
$$

and, as a consequence,

$$
\begin{aligned}
N(p, \alpha, \psi) & =\alpha \frac{\partial \psi}{\partial p}(\nabla p \times \nabla \alpha) \cdot \nabla p \\
& +\alpha \frac{\partial \psi}{\partial \alpha}(\nabla p \times \nabla \alpha) \cdot \nabla \alpha \\
& =0
\end{aligned}
$$

and

$$
(\nabla p \times \nabla \alpha) \cdot \nabla p=(\nabla p \times \nabla \alpha) \cdot \nabla \alpha=0 .
$$

The volume $V$ of a $(p, \alpha, \psi)$ unit cell becomes infinite and the cells degenerate into solenoids; the $(p, \alpha)$ solenoid, the $(p, \psi)$ solenoid, and the $(\psi, \alpha)$ solenoid have the same direction at each point, a direction which, however, can be different for different points.

The vorticity theorem (1.40) then becomes a hydrodynamical conservation theorem

$$
\frac{d}{d t}\{\alpha(\nabla \times \mathbf{v}+2 \boldsymbol{\Omega}) \cdot \nabla \psi\}=0
$$

the derivation of which I have recently given in another way $^{3}$. The adiabatic motion in the atmosphere (as a special case of the polytropic case) satisfies

$$
\frac{d}{d t}\{\alpha(\nabla \times \mathbf{v}+2 \boldsymbol{\Omega}) \cdot \nabla \theta\}=0
$$

Here,

$$
\theta=T\left(\frac{1000}{p}\right)^{\kappa}=\frac{1000^{\kappa}}{R} p^{1-\kappa} \alpha
$$

is the potential temperature ( $R=$ gas constant, $\kappa=$ $\left.R / c_{p}\right)$. The adiabatic special case of the new vorticity theorem, that is (1.46), may prove to be a very useful tool for isentropic analysis (C. G. Rossby).

Here let us discuss as an application of (1.46), the beginning of an adiabatic vertical motion along the vertical axis of expansion or contraction of a volume of air. If the positive $z$ axis is upward and if $(x, y, z)$ is a right-hand cartesian system, the horizontal $(x, y)$ surface touches the earth's surface at latitude $\varphi$ and

$$
\mathbf{k} \cdot \nabla \times \mathbf{v}=\frac{\partial v}{\partial x}-\frac{\partial u}{\partial y}=\zeta
$$

$(\zeta>0$ for cyclonic motion and $\zeta<0$ for anticyclonic motion) and

$$
\begin{array}{ll}
\mathbf{k} \cdot \Omega=\Omega \sin \varphi>0 & \text { (for the northern } \\
& \text { hemisphere) }
\end{array}
$$

The conservation theorem (1.46) gives

$$
\frac{d}{d t}\left\{\alpha(\zeta+2 \Omega \sin \varphi) \frac{\partial \theta}{\partial z}\right\}=0,
$$

where

$$
\frac{d}{d t}=\frac{\partial}{\partial t}+w \frac{\partial}{\partial z}
$$


because $u=v=0$ at the vertical axis (however, $\partial u / \partial x, \partial u / \partial y, \partial v / \partial x, \partial v / \partial y \neq 0$ ). From (1.50) we obtain

$$
\begin{aligned}
& \frac{d}{d t}(\zeta+2 \Omega \sin \varphi)= \\
& \quad-(\zeta+2 \Omega \sin \varphi)\left(\alpha \frac{\partial \theta}{\partial z}\right)^{-1} \frac{d}{d t}\left(\alpha \frac{\partial \theta}{\partial z}\right) .
\end{aligned}
$$

At the initial time, we assume $\zeta=0$; also in this case, $d(2 \Omega \sin \varphi) / d t=0$, so that from (1.52)

$$
\frac{d \zeta}{d t}=-2 \Omega \sin \varphi\left(\alpha \frac{\partial \theta}{\partial z}\right)^{-1} \frac{d}{d t}\left(\alpha \frac{\partial \theta}{\partial z}\right) .
$$

Now ${ }^{4}$,

$$
\left(\alpha \frac{\partial \theta}{\partial z}\right)^{-1} \frac{d}{d t}\left(\alpha \frac{\partial \theta}{\partial z}\right)=\left(\frac{\partial u}{\partial x}+\frac{\partial v}{\partial y}\right)_{\theta}
$$

where $(\partial u / \partial x+\partial v / \partial y)_{\theta}$ is the divergence on an isentropic surface. Thus, from (1.53) and (1.54) it follows that

$$
\frac{d \zeta}{d t}=-2 \Omega \sin \varphi\left(\frac{\partial u}{\partial x}+\frac{\partial v}{\partial y}\right)_{\theta}
$$

With this we get for convergence, $\partial u / \partial x+\partial v / \partial y<0$, the development of cyclonic vorticity, while for divergence, $\partial u / \partial x+\partial v / \partial y>0$, the development of anticyclonic vorticity. Since $\alpha(\partial \theta / \partial z)=(1 / \rho)(\partial \theta / \partial z)$ is a measure of the vertical stability of an air mass, we can develop the following interpretation: In an air mass without relative vorticity, anticyclonic (cyclonic) vorticity will be developed by adiabatic vertical motion if the stability $\alpha \partial \theta / \partial z$ increases (decreases).

The vorticity equation (1.40) can also be written in integral form. Multiplying by the mass element $\rho d \tau(d \tau=$ volume element $)$ and integrating over the total mass $m=\iiint \rho d \tau$, we obtain ${ }^{5}$

$$
\begin{aligned}
\iiint \rho \frac{d}{d t}(\alpha \mathbf{Z} \cdot \nabla \psi) d \tau & =\frac{d}{d t} \iiint \mathbf{Z} \cdot \nabla \psi d \tau \\
& =N_{m}(p, \alpha, \psi)
\end{aligned}
$$

where $N_{m}(p, \alpha, \psi)$ is the number of $(p, \alpha, \psi)$ unit cells contained in the mass $m$. Since $\mathbf{Z} \cdot \nabla \psi=\nabla \cdot(\psi \mathbf{Z})$, Gauss' theorem allows us to write (1.56) as

$$
\frac{d}{d t} \iint Z_{n} \psi d \sigma=N_{m}(p, \alpha, \psi)
$$

where $d \sigma$ is an area element of the closed surface and $n$ denotes the outward normal.
With a non-conservative quantity $\psi^{*}$ (i.e., $d \psi^{*} / d t \neq$ $0)$ one obtains the new vorticity theorem ${ }^{6}$ in its general form since one writes $d \psi^{*} / d t-\partial \psi^{*} / \partial t$ instead of $\mathbf{v} \cdot \nabla \psi$ from (1.17) on. This generalized vorticity theorem takes the form

$$
\frac{d}{d t}\left(\alpha \mathbf{Z} \cdot \nabla \psi^{*}\right)=N\left(p, \alpha, \psi^{*}\right)+\alpha \mathbf{Z} \cdot \nabla \frac{d \psi^{*}}{d t}
$$

which can be expressed as follows:

In an ideal fluid, the individual time change of the specific volume multiplied by the scalar product of the absolute vorticity vector and the gradient of a field function is equal to the number of $\left(p, \alpha, \psi^{*}\right)$ unit cells plus the gradient of $d \psi^{*} / d t$ projected onto the vector $\alpha \mathbf{Z}$.

From (1.57) one obtains, for example, the generalized Helmholtz vorticity equation as a special case if one gives instead of $\psi^{*}$ the components of the position vector $\mathbf{r}=(x, y, z)$.

The integral form of (1.57) is

$$
\begin{aligned}
\frac{d}{d t} \iint Z_{n} \psi^{*} d \sigma & =N_{m}(p, \alpha, \psi) \\
& +\iint Z_{n} \frac{d \psi^{*}}{d t} d \sigma
\end{aligned}
$$

where $d \sigma$ is an area element ( $n=$ outward normal) of the volume $\tau . N_{m}\left(p, \alpha, \psi^{*}\right)$ has the same meaning as above; the derivation makes use of $\mathbf{Z} \cdot \nabla\left(d \psi^{*} / d t\right)=$ $\nabla \cdot\left[\mathbf{Z}\left(d \psi^{*} / d t\right)\right]$.

\section{References}

$\left.{ }^{1}\right)$ R. Emden, Thermodynamik der Himmelskörper. Enzykl. Math. Wiss., VI, 2, 24. Leipzig-Berlin 1926, S. 389.

$\left.{ }^{2}\right)$ V. Bjerknes u. Mitarbeiter, Physikalische Hydrodynamik. Berlin 1933, S. 1-8.

$\left.{ }^{3}\right)$ H. Ertel, Ein neuer hydrodynamischer Erhaltungssatz. Die Naturwissenschaften, Berlin 1942.

$\left.{ }^{4}\right)$ H. Ertel, Über die individuelle Änderung des vertikalen Temperaturgradienten in der Atmosphäre. Met. Z. 1941, S. 223-236.

5) H. Ertel, Methoden und Probleme der Dynamischen Meteorologie. Berlin 1938, S. 21.

$\left.{ }^{6}\right)$ H. Ertel, Über hydrodynamische Wirbelgleichungen. Phys. Z. 1942. 


\section{ERTEL (1942b): A new hydrodynamical conservation principle}

In a compressible fluid whose particles move polytropically, the specific volume $\alpha$ can be expressed as a function of the pressure $p$ and the "polytropic temperature" $\Theta_{s}=T\left(\rho_{0} / \rho\right)^{1 / s}\left(s=\left(c_{v}-\beta\right) /\left(c_{p}-c_{v}\right)\right.$ is the polytropic order, $\beta=d Q / d T$ the constant polytropic specific heat capacity, $\rho=1 / \alpha$ the density, and $\left.\rho_{0}=1 \mathrm{~g} / \mathrm{cm}^{3}\right)^{1}$ :

$$
\alpha=\frac{1}{\rho}=\psi\left(p, \Theta_{s}\right)
$$

In general, $\Theta_{s}$ varies from particle to particle but remains constant in time for a given particle:

$$
\frac{d \Theta_{s}}{d t}=\frac{\partial \Theta_{s}}{\partial t}+\mathbf{v} \cdot \nabla \Theta_{s}=\frac{\partial \Theta_{s}}{\partial t}+v_{k} \frac{\partial \Theta_{s}}{\partial x_{k}}=0,
$$

the last part of which makes use of the usual summation convention. With respect to a rotating rectangular cartesian coordinate system $\left(x_{i}, i=1,2,3\right)$ whose rotation is described by the constant rotation vector $\Omega$, the hydrodynamical vorticity equation ${ }^{2}$ can be written

$$
\frac{d}{d t}\left(\frac{\xi_{i}}{\rho}\right)-\left(\frac{\xi_{k}}{\rho}\right) \frac{\partial v_{i}}{\partial x_{k}}=-\frac{1}{\rho} \nabla \times\left(\frac{1}{\rho} \nabla p\right),
$$

where

$$
\boldsymbol{\xi}=\nabla \times \mathbf{v}+2 \boldsymbol{\Omega}
$$

denotes the absolute (relative to an inertial system) vorticity vector and where $\mathbf{v}=v_{i}(i=1,2,3)$ denotes the wind relative to the rotating $x_{i}$ system.

On account of (2.1),

$$
\nabla \times\left(\frac{1}{\rho} \nabla p\right)=\frac{\partial \psi}{\partial \Theta_{s}} \nabla \Theta_{s} \times \nabla p
$$

or in component form

$$
\left[\nabla \times\left(\frac{1}{\rho} \nabla p\right)\right]_{i}=\frac{\partial \psi}{\partial \Theta_{s}} \epsilon_{i j k} \frac{\partial \Theta_{s}}{\partial x_{j}} \frac{\partial p}{\partial x_{k}},
$$

where $\epsilon_{i j k}$ denotes the following tensor:

$$
\epsilon_{i j k}=\left\{\begin{aligned}
1 & \text { for a cyclic sequence of indices } \\
-1 & \text { for a non-cyclic sequence of indices } \\
0 & \text { if any two indices agree }
\end{aligned}\right.
$$

The vorticity equation (2.3) can now be written $(\xi=$ $\left.\xi_{i}, i=1,2,3\right)$

$$
\frac{d}{d t}\left(\alpha \xi_{i}\right)-\left(\alpha \xi_{k}\right) \frac{\partial v_{i}}{\partial x_{k}}=-\alpha \frac{\partial \psi}{\partial \Theta_{s}} \epsilon_{i j k} \frac{\partial \Theta_{s}}{\partial x_{j}} \frac{\partial p}{\partial x_{k}} .
$$

Multiplying by $\partial \Theta_{s} / \partial x_{r}$ and making use of

$$
\epsilon_{i j k} \frac{\partial \Theta_{s}}{\partial x_{i}} \frac{\partial \Theta_{s}}{\partial x_{j}} \frac{\partial p}{\partial x_{k}}=0
$$

it follows from (2.5) that

$$
\frac{\partial \Theta_{s}}{\partial x_{i}} \frac{d}{d t}\left(\alpha \xi_{i}\right)-\left(\alpha \xi_{k}\right) \frac{\partial v_{i}}{\partial x_{k}} \frac{\partial \Theta_{s}}{\partial x_{i}}=0,
$$

or in another form

$$
\begin{aligned}
\frac{d}{d t}\left(\alpha \frac{\partial \Theta_{s}}{\partial x_{i}} \xi_{i}\right) & -\left(\alpha \xi_{i}\right) \frac{d}{d t}\left(\frac{\partial \Theta_{s}}{\partial x_{i}}\right) \\
& -\left(\alpha \xi_{k}\right) \frac{\partial v_{i}}{\partial x_{k}} \frac{\partial \Theta_{s}}{\partial x_{i}}=0 .
\end{aligned}
$$

From (2.2) we now obtain

$$
\frac{\partial}{\partial x_{i}}\left(\frac{d \Theta_{s}}{d t}\right)=\frac{d}{d t}\left(\frac{\partial \Theta_{s}}{\partial x_{i}}\right)+\frac{\partial \Theta_{s}}{\partial x_{k}} \frac{\partial v_{k}}{\partial x_{i}}=0,
$$

so that

$$
\xi_{i} \frac{d}{d t}\left(\frac{\partial \Theta_{s}}{\partial x_{i}}\right)=-\xi_{i} \frac{\partial \Theta_{s}}{\partial x_{k}} \frac{\partial v_{k}}{\partial x_{i}}=-\xi_{k} \frac{\partial \Theta_{s}}{\partial x_{i}} \frac{\partial v_{i}}{\partial x_{k}},
$$

and the substitution of (2.9) into (2.7) leads to the following conservation principle:

$$
\begin{aligned}
\frac{d}{d t}\left(\alpha \xi_{i} \frac{\partial \Theta_{s}}{\partial x_{i}}\right) & =\frac{d}{d t}\left\{\alpha(\nabla \times \mathbf{v}+2 \Omega)_{n} \nabla_{n} \Theta_{s}\right\} \\
& =0
\end{aligned}
$$

where $n$ denotes the direction normal to the planes of constant polytropic temperature and toward increasing $\Theta_{s}$. Equation (2.10) is the analytical version of the following conservation principle ${ }^{3}$ :

In a fluid which moves polytropically, the quantity formed by multiplying the specific volume by the scalar product of the absolute vorticity vector and the gradient of the polytropic temperature is conserved following individual particles.

For $s=c_{v} /\left(c_{p}-c_{v}\right)$, the polytropic temperature reduces to the potential temperature

$$
\theta=T\left(\frac{P}{p}\right)^{\kappa}
$$

( $P=$ reference pressure, e.g. $1000 \mathrm{mb}$, and $\kappa=R / c_{p}$ ) and (2.2) becomes

$$
\frac{d \theta}{d t}=0
$$


The conservation principle then becomes

$$
\frac{d}{d t}\left\{\alpha(\nabla \times \mathbf{v}+2 \boldsymbol{\Omega})_{n} \nabla_{n} \theta\right\}=0
$$

which in meteorology is connected to the isentropic analysis work of C. G. Rossby.

\section{References}

1) R. EMdEN, Thermodynamik der Himmelskörper.
Enzykl. Math. Wiss., VI, 2, 24, S. 389.

Berlin-Leipzig 1926.

2) W. Voigt, Kompendium der theoretischen Physik I, 270.-Leipzig 1895.

${ }^{3}$ ) The conservation principle given here can be regarded as a special case of the new hydrodynamical vorticity equation which $I$ have proven in another paper.

(Meteorol. Z. 1942).

\section{ERTEL (1942c): On hydrodynamical vorticity equations}

\section{Introduction}

Adopting the usual summation convention (according to which every index occurring twice in a single term is summed from 1 to 3 ) the vorticity equation for an ideal compressible fluid reads as follows:

$$
\frac{d}{d t}\left(\alpha \zeta_{i}\right)-\left(\alpha \zeta_{k}\right) \frac{\partial w_{i}}{\partial z_{k}}=\epsilon_{i j k} \alpha \frac{\partial p}{\partial z_{j}} \frac{\partial \alpha}{\partial z_{k}},
$$

where $\alpha=\rho^{-1}$ is the specific volume, $p$ the pressure, $z_{i}(i=1,2,3)$ the rectangular cartesian (inertial) coordinate system, $w_{i}(i=1,2,3)$ the wind components, $\zeta_{i}=\epsilon_{i j k}\left(\partial w_{k} / \partial z_{j}\right)=(\nabla \times \mathbf{w})_{i}$, and

$$
\epsilon_{i j k}=\left\{\begin{aligned}
1 & \text { for a cyclic sequence of indices, } \\
-1 & \text { for a non-cyclic sequence of indices } \\
0 & \text { if any two indices agree. }
\end{aligned}\right.
$$

Through a generalization of (3.1) we shall now derive a new vorticity equation.

\section{General formulation of hydrodynamical vorticity equations by means of a commutator relation}

In order to treat the most general case we refer not to the inertial system as in (3.1) but rather to a rotating rectangular coordinate system $x_{i}$, whose rotation is described by the constant rotation vector $f_{i}(i=1,2,3)$; the vorticity equations then take the form

$$
\frac{d}{d t}\left(\alpha \xi_{i}\right)-\left(\alpha \xi_{k}\right) \frac{\partial v_{i}}{\partial x_{k}}=\epsilon_{i j k} \alpha \frac{\partial p}{\partial x_{j}} \frac{\partial \alpha}{\partial x_{k}}
$$

with the individual (material) derivative operator

$$
\frac{d}{d t}=\frac{\partial}{\partial t}+v_{k} \frac{\partial}{\partial x_{k}}
$$

and the absolute vorticity

$$
\xi_{i}=\epsilon_{i j k} \frac{\partial v_{k}}{\partial x_{j}}+2 f_{i},
$$

in which $v_{i}(i=1,2,3)$ is the wind component and $\epsilon_{i j k}\left(\partial v_{k} / \partial x_{j}\right)$ is the vorticity relative to the rotating $x_{i}$ system. The existence of a potential for other forces is implicit in (3.2).

Scalar multiplication of (3.2) by the gradient $\partial \psi / \partial x_{i}$ of a hydrodynamical field function $\psi$ (scalar, vector or tensor component) leads easily to the form

$$
\begin{aligned}
\frac{d}{d t}\left(\alpha \xi_{i} \frac{\partial \psi}{\partial x_{i}}\right) & -\left(\alpha \xi_{i}\right) \frac{d}{d t}\left(\frac{\partial \psi}{\partial x_{i}}\right)-\left(\alpha \xi_{k}\right) \frac{\partial \psi}{\partial x_{i}} \frac{\partial v_{i}}{\partial x_{k}} \\
& =\alpha \epsilon_{i j k} \frac{\partial \psi}{\partial x_{i}} \frac{\partial p}{\partial x_{j}} \frac{\partial \alpha}{\partial x_{k}}
\end{aligned}
$$

which, through the exchange of the summation indices in the third term on the left side, can be written

$$
\begin{aligned}
\frac{d}{d t}\left(\alpha \xi_{i} \frac{\partial \psi}{\partial x_{i}}\right) & -\left(\alpha \xi_{i}\right)\left\{\frac{d}{d t}\left(\frac{\partial \psi}{\partial x_{i}}\right)+\frac{\partial \psi}{\partial x_{k}} \frac{\partial v_{k}}{\partial x_{i}}\right\} \\
& =\alpha \epsilon_{i j k} \frac{\partial \psi}{\partial x_{i}} \frac{\partial p}{\partial x_{j}} \frac{\partial \alpha}{\partial x_{k}} .
\end{aligned}
$$

Now, from (3.3), we have

$$
\begin{aligned}
\frac{d}{d t}\left(\frac{\partial \psi}{\partial x_{i}}\right) & =\frac{\partial^{2} \psi}{\partial x_{i} \partial t}+v_{k} \frac{\partial^{2} \psi}{\partial x_{i} \partial x_{k}} \\
& =\frac{\partial}{\partial x_{i}}\left(\frac{d \psi}{d t}\right)-\frac{\partial \psi}{\partial x_{k}} \frac{\partial v_{k}}{\partial x_{i}}
\end{aligned}
$$

with which (3.5) can be reduced to

$$
\begin{aligned}
\frac{d}{d t}\left(\alpha \xi_{i} \frac{\partial \psi}{\partial x_{i}}\right) & -\left(\alpha \xi_{i} \frac{\partial}{\partial x_{i}}\right) \frac{d \psi}{d t} \\
& =\alpha \epsilon_{i j k} \frac{\partial \psi}{\partial x_{i}} \frac{\partial p}{\partial x_{j}} \frac{\partial \alpha}{\partial x_{k}}
\end{aligned}
$$


whose right hand side has the following simple meaning: Assume that the surfaces $p=$ const, $\alpha=$ const and $\psi=$ const (with scalar value differences of 1 ) are constructed throughout space; each set of surfaces divides space into unit layers. As long as there is no homogeneous relation $F(p, \alpha)=0\left(\mathrm{~V}\right.$. Bjerknes $\left.{ }^{1}\right)$ between $p$ and $\alpha$, the intersections of the $p$ and $\alpha$ unit layers form $(p, \alpha)$ unit solenoids. The $(p, \alpha)$ unit solenoids are intersected by the $\psi$ unit layers, as long as $\psi$ is not of the form $\psi=\psi(p, \alpha)$. In this way a field of $(p, \alpha, \psi)$ unit cells is formed. We can then interpret

$$
\epsilon_{i j k} \frac{\partial \psi}{\partial x_{i}} \frac{\partial p}{\partial x_{j}} \frac{\partial \alpha}{\partial x_{k}}=(\nabla p \times \nabla \alpha) \cdot \nabla \psi= \pm \frac{1}{V}
$$

as the reciprocal volume $V$ of a $(p, \alpha, \psi)$ unit cell or the number of $(p, \alpha, \psi)$ unit cells in a unit volume. The proof is accomplished by integration of $(\nabla p \times \nabla \alpha)$. $\nabla \psi=\nabla \cdot(\psi \nabla p \times \nabla \alpha)$ over the volume of a unit cell and application of Gauss' integral theorem; the portion of the surface integral over the four sides of the $(p, \alpha)$ solenoids disappears, and the remainder yields a value of +1 or -1 , depending on whether the three gradients $\nabla p, \nabla \alpha, \nabla \psi$ are oriented in the sense of a right-handed or left-handed screw. (For a related discussion see H. $\mathrm{Ertel}^{2}$ ). Thus, we can interpret

$$
\begin{aligned}
\alpha \epsilon_{i j k} \frac{\partial \psi}{\partial x_{i}} \frac{\partial p}{\partial x_{j}} \frac{\partial \alpha}{\partial x_{k}} & =\alpha(\nabla p \times \nabla \alpha) \cdot \nabla \psi \\
& = \pm \frac{\alpha}{V}=N(p, \alpha, \psi)
\end{aligned}
$$

as the (positive or negative) number $N(p, \alpha, \psi)$ of $(p, \alpha, \psi)$ unit cells in the specific volume; moreover it follows from (3.7) that

$$
\left.\begin{array}{l}
N(p, \alpha, \psi)=N(\psi, p, \alpha)=N(\alpha, \psi, p) \\
N(p, \alpha, \psi)=-N(\alpha, p, \psi)=-N(\psi, \alpha, p)
\end{array}\right\} .
$$

Furthermore $N(p, \alpha, \psi)=0$ for homogeneous fuids $(\nabla \rho=0)$ while for inhomogeneous fluids $(\nabla \rho \neq 0)$

$$
N(p, \alpha, \psi)=0, \text { if }\left\{\begin{array}{l}
\psi=\psi(p) \\
\psi=\psi(\alpha) \\
\psi=\psi(p, \alpha) \\
\alpha=\alpha(p)
\end{array}\right\} .
$$

Combining (3.6) and (3.8) we obtain

$$
\frac{d}{d t}\left(\alpha \xi_{i} \frac{\partial \psi}{\partial x_{i}}\right)-\left(\alpha \xi_{i} \frac{\partial}{\partial x_{i}}\right) \frac{d \psi}{d t}=N(p, \alpha, \psi)
$$

a generalized formulation for hydrodynamical vorticity equations in the commutator relation form

$$
\left(D_{1} D_{2}-D_{2} D_{1}\right) \psi=N(p, \alpha, \psi),
$$

where the operators

$$
D_{1}=\frac{d}{d t}=\frac{\partial}{\partial t}+v_{k} \frac{\partial}{\partial x_{k}}=\frac{\partial}{\partial t}+(\mathbf{v} \cdot \nabla)
$$

and

$$
D_{2}=\alpha \xi_{i} \frac{\partial}{\partial x_{i}}=\alpha \xi_{k} \frac{\partial}{\partial x_{k}}=\alpha(\nabla \times \mathbf{v}+2 \mathbf{f}) \cdot \nabla
$$

are in general not commutative.

Multiplying (3.11) by the mass element $\rho d \tau(d \tau=$ volume element), integrating over the volume $\tau$ containing the total mass $m=\iiint \rho d \tau$, using $\alpha \rho=1$, and noting that

$$
\begin{aligned}
\iiint \rho \frac{d}{d t}\left(\alpha \xi_{i} \frac{\partial \psi}{\partial x_{i}}\right) d \tau & =\frac{D}{D t} \iiint \xi_{i} \frac{\partial \psi}{\partial x_{i}} d \tau \\
& =\frac{D}{D t} \iiint \frac{\partial\left(\psi \xi_{i}\right)}{\partial x_{i}} d \tau \\
& =\frac{D}{D t} \iint \psi \xi_{n} d \Omega
\end{aligned}
$$

as well as

$$
\begin{aligned}
\iiint \xi_{i} \frac{\partial}{\partial x_{i}}\left(\frac{d \psi}{d t}\right) d \tau & =\iiint \frac{\partial}{\partial x_{i}}\left(\xi_{i} \frac{d \psi}{d t}\right) d \tau \\
& =\iint \xi_{n} \frac{d \psi}{d t} d \Omega
\end{aligned}
$$

yields in the integral form $(d \Omega=$ surface element of $\tau$; $n=$ outward normal)

$$
\frac{D}{D t} \iint \xi_{n} \psi d \Omega-\iint \xi_{n} \frac{d \psi}{d t} d \Omega=N_{m}(p, \alpha, \psi),
$$

where $N_{m}(p, \alpha, \psi)=\iiint \rho N(p, \alpha, \psi) d \tau$ is the number of $(p, \alpha, \psi)$ unit cells contained in the total mass $m$ and $\frac{D}{D t}$ denotes the individual time derivative of the entire moving mass; in this derivation we note that for any $F(F=$ scalar, vector- or tensor-component $)$ we have

$$
\begin{aligned}
\frac{D}{D t} \iiint \rho F d \tau & =\iiint \frac{\partial(\rho F)}{\partial t} d \tau+\iint \rho v_{n} F d \Omega \\
& =\iiint \rho \frac{d F}{d t} d \tau,
\end{aligned}
$$

since, by means of the continuity equation

$$
\frac{\partial \rho}{\partial t}+\nabla \cdot(\rho \mathbf{v})=0
$$


the following transformation is possible:

$$
\begin{aligned}
\iiint \frac{\partial(\rho F)}{\partial t} d \tau & +\iint \rho v_{n} F d \Omega \\
& =\iiint\left\{\frac{\partial(\rho F)}{\partial t}+\nabla \cdot(\rho \mathbf{v} F)\right\} d \tau \\
& =\iiint \rho\left\{\frac{\partial F}{\partial t}+(\mathbf{v} \cdot \nabla) F\right\} d \tau
\end{aligned}
$$

Equation (3.18) with $F=\alpha \xi_{i}\left(\partial \psi / \partial x_{i}\right)$ reverts back to (3.15) when we take into consideration the fact that $\xi_{i}$ represents a nondivergent vector, i.e.

$$
\frac{\partial \xi_{i}}{\partial x_{i}}=0
$$

\section{Conclusions}

A. If one substitutes for $\psi$ in (3.11) or (3.12) the components $x_{s}(s=1,2,3)$ of the position vector $\mathbf{r}$ of a fluid particle, one obtains, because $D_{1} x_{s}=v_{s}$, the vorticity equation (3.2), which for a homogeneous and incompressible fluid, turns into the Helmholtz vorticity equation in the rotating system.

B. If $\psi$ is a conservative property of a flid particle,

$$
D_{1} \psi=\frac{d \psi}{d t}=0
$$

and it then follows from (3.11) or (3.12) that

$$
\frac{d}{d t}\left(\alpha \xi_{i} \frac{\partial \psi}{\partial x_{i}}\right)=N(p, \alpha, \psi)
$$

which can be stated as follows:

For a fluid variable $\psi$ which is individually conserved but has spatial variability, the individual time change of the specific volume multiplied by the scalar product of the absolute vorticity vector with the gradient of $\psi$ is equal to the number of $(p, \alpha, \psi)$ unit cells contained in the specific volume $\alpha$.

C. If $\psi$ is a conserved quantity and at the same time has the form

$$
\psi=\psi(p, \alpha),
$$

for all fluid particles, then, because

$$
\nabla \psi=\frac{\partial \psi}{\partial p} \nabla p+\frac{\partial \psi}{\partial \alpha} \nabla \alpha
$$

(3.10) yields $N(p, \alpha, \psi)=0$ and (3.22) reduces to

$$
\frac{d}{d t}\left(\alpha \xi_{i} \frac{\partial \psi}{\partial x_{i}}\right)=0
$$

which is an analytical statement of the following conservation principle:

For a fluid variable $\psi$ which is individually conserved but has a spatial variability such that it depends only on pressure and specific volume, the individual time change of the specific volume multiplied by the scalar product of the absolute vorticity vector with the gradient of $\psi$ vanishes.

An example of a function $\psi$ which satisfies conditions (3.21) and (3.23) is the entropy of an ideal gas which moves adiabatically; another example is the polytropic temperature ${ }^{3}$ of an ideal gas which moves polytropically; the conservation principle is therefore valid for adiabatic or polytropic currents in ideal gases with spatially variable entropy or polytropic temperature (the earth's atmosphere, stellar atmospheres).

D. A homogeneous fluid is defined by $\nabla \alpha=\nabla(1 / \rho)=$ 0 , from which (using (3.8)) it follows that $N(p . \alpha, \psi)$ vanishes for any function $\psi$; furthermore, $N(p, \alpha, \psi)$ vanishes for any function $\psi$ in the case of barotropy, i.e. $\alpha=\alpha(p)$. Then, there follows from (3.12) the principle:

For homogeneous fluids and for inhomogeneous barotropic fluids the operators $D_{1}$ and $D_{2}$ are always commutative; on the other hand, for inhomogeneous baroclinic fluids, the operators $D_{1}$ and $D_{2}$ are only commutative for those functions $\psi$ which cause the determinant

$$
\frac{\partial(p, \alpha, \psi)}{\partial\left(x_{1}, x_{2}, x_{3}\right)}=(\nabla p \times \nabla \alpha) \cdot \nabla \psi
$$

to vanish.

\section{References}

1) V. Bjerknes und Mitarbeiter, Physikalische Hydrodynamik, S. 3ff., Berlin 1933.

2) H. Ertel, Meteorol. Zeitschr., 59, 1942.

3) R. Emden, Enzykl. Math. Wiss., VI, 2, 24, Leipzig und Berlin 1926. 


\section{ERTEL (1942d): On the relationship between the new hydrodynamic vorticity theorem and Bjerknes' circulation theorem}

\section{Summary}

It is proven that Bjerknes' circulation theorem is a special case of the new hydrodynamical vorticity theorem.

\section{The new vorticity theorem}

Recently I derived a very general hydrodynamic vorticity theorem [1] [2]. In its differential form it reads as follows:

$$
\frac{d}{d t}(\sigma \mathfrak{W} \cdot \nabla \psi)-(\sigma \mathfrak{W} \cdot \nabla) \frac{d \psi}{d t}=N(p, \sigma, \psi) .
$$

It has the form of a commutation relation

$$
\left(D_{1} D_{2}-D_{2} D_{1}\right) \psi=N(p, \sigma, \psi)
$$

with the generally noncommutative operators

$$
D_{1}=\frac{d}{d t}=\frac{\partial}{\partial t}+\mathbf{v} \cdot \nabla
$$

and

$$
D_{2}=(\sigma \mathfrak{W} \cdot \nabla)=\sigma(\operatorname{rot} \mathbf{v}+2 \mathbf{f}) \cdot \nabla,
$$

where the symbols used in (4.1) through (4.4) are defined as follows: $\sigma=$ specific volume $=1 / \rho(\rho=$ density); $p=$ pressure; $\mathbf{v}=$ velocity vector relative to a coordinate system, its rotation described through the constant rotation vector $\mathbf{f} ; \mathfrak{W}=\operatorname{rot} \mathbf{v}+2 \mathbf{f}=$ (absolute) vorticity; $\psi=$ hydrodynamic field function (scalar, vector, or tensor component); $N(p, \sigma, \psi)=$ $\sigma[\nabla p, \nabla \sigma] \cdot \nabla \psi=$ number of $(p, \sigma, \psi)$-cells contained within a specific volume $\sigma$.

For the derivation of (4.1), it has been assumed that the fluid is ideal and that external forces acting on the fluid possess a potential. The sign of $N(p, \sigma, \psi)$ is positive or negative depending on whether the generally nonorthogonal vectors $\nabla p, \nabla \sigma$ and $\nabla \psi$ are oriented in a right- or left-handed system.

One can obtain equations for each component of vorticity by specifying in (4.1) the function $\psi$ to have a purely geometric interpretation. Namely, one successively sets $\psi=x, \psi=y$, and $\psi=z$ in such a way that from (4.1) the following vorticity equations result:

$$
\left.\begin{array}{rl}
\frac{d}{d t}\left(\sigma \mathfrak{W}_{x}\right)-(\sigma \mathfrak{W} \cdot \nabla) v_{x} & =\sigma[\nabla p, \nabla \sigma]_{x}, \\
\frac{d}{d t}\left(\sigma \mathfrak{W}_{y}\right)-(\sigma \mathfrak{W} \cdot \nabla) v_{y} & =\sigma[\nabla p, \nabla \sigma]_{y}, \\
\frac{d}{d t}\left(\sigma \mathfrak{W}_{z}\right)-(\sigma \mathfrak{W} \cdot \nabla) v_{z} & =\sigma[\nabla p, \nabla \sigma]_{z},
\end{array}\right\}
$$

where, for instance,

$$
[\nabla p, \nabla \sigma]_{x}=\frac{\partial p}{\partial y} \frac{\partial \sigma}{\partial z}-\frac{\partial p}{\partial z} \frac{\partial \sigma}{\partial y}
$$

is the number of the isobar-isostere unit solenoids per unit surface area normal to the $+x$ direction. For irrotational coordinates $(f \equiv 0)$ and barotropy ( $p=$ $p(\sigma)$ ), (4.5) yields the vorticity equations of E. J. NANSON (1874); furthermore, adding incompressibility, one obtains the vorticity equations of HELMHOLTZ (1858) [LAGRANGE (1781), CAUCHY (1827), STOKES (1848)].

The integral form of the new vorticity theorem reads (Ertel, 1.c.):

$$
\frac{d}{d t} \iint \mathfrak{W}_{n} \psi d \Omega-\iint \mathfrak{W}_{n} \frac{d \psi}{d t} d \Omega=N_{m}(p, \sigma, \psi) .
$$

Here,

$$
\begin{aligned}
N_{m}(p, \sigma, \psi) & =\iiint \rho N(p, \sigma, \psi) d \tau \\
& =\iiint[\nabla p, \nabla \sigma] \cdot \nabla \psi d \tau
\end{aligned}
$$

is the number of $(p, \sigma, \psi)$-unit cells, $m=\iiint \rho d \tau$ is the mass (with volume $\tau$ contained in the surface $\Omega$ ), and $n$ in (4.7) represents the outward normal. Because

$$
[\nabla p, \nabla \sigma] \cdot \nabla \psi=\nabla \cdot(\psi[\nabla p, \nabla \sigma]),
$$

(4.8) can be transformed through Gauss' integral theorem into

$$
N_{m}(p, \sigma, \psi)=\iint \psi[\nabla p, \nabla \sigma]_{n} d \Omega
$$

Then, the integral form (4.7) of the new vorticity theorem changes into

$$
\begin{aligned}
\frac{d}{d t} \iint \mathfrak{W}_{n} \psi d \Omega & -\iint \mathfrak{W}_{n} \frac{d \psi}{d t} d \Omega \\
& =\iint \psi[\nabla p, \nabla \sigma]_{n} d \Omega .
\end{aligned}
$$

One assumes in (4.7) and (4.10) that the functions $\psi$, $d \psi / d t, \mathfrak{W}$ and $[\nabla p, \nabla \sigma]$ are defined on the surface $\Omega$ and in the volume $\tau$ the first derivatives are continuous 
with respect to the spatial coordinates; the closed surface $\Omega$ can itself consist of finitely many pieces of continuous tangential planes.

\section{The new vorticity theorem with a discontinuous $\psi$-function}

Consider discontinuous $\psi$ and $d \psi / d t$ on the surface $\Sigma$. We denote the jump in $\psi$ as

$$
\{\psi\}=\psi_{+0}-\psi_{-0}
$$

and the jump in $d \psi / d t$ as

$$
\left\{\frac{d \psi}{d t}\right\}=\left(\frac{d \psi}{d t}\right)_{+0}-\left(\frac{d \psi}{d t}\right)_{-0}
$$

on the surface $\Sigma$ (the indices +0 and -0 specify the function values directly above and directly below the surface $\Sigma$, respectively). Then, we construct a cylinder with face $F$ parallel to $\Sigma$ and the infinitesmal height $h$. Applying the integral form (4.10) of the new vorticity theorem to the region $F \cdot h$ and taking the limit as $h \rightarrow 0$, the integral over the lateral surface vanishes and we obtain the following:

$$
\begin{gathered}
\frac{d}{d t} \iint \mathfrak{W}_{n} \psi_{-0} d F-\iint \mathfrak{W}_{n}\left(\frac{d \psi}{d t}\right)_{+0} d F \\
+\frac{d}{d t} \iint \mathfrak{W}_{-n} \psi_{-0} d F-\iint \mathfrak{W}_{-n}\left(\frac{d \psi}{d t}\right)_{-0} d F \\
=\iint \psi_{+0}[\nabla p, \nabla \sigma]_{n} d F \\
+\iint \psi_{-0}[\nabla p, \nabla \sigma]_{-n} d F
\end{gathered}
$$

By the continuity of $\mathfrak{W}$ and $[\nabla p, \nabla \sigma]$ on $\Sigma$ (and thus on the subarea $F$ of $\Sigma$ )

$$
\mathfrak{W}_{-n}=-\mathfrak{W}_{n}
$$

and

$$
[\nabla p, \nabla \sigma]_{-n}=[\nabla p, \nabla \sigma]_{n} .
$$

Then, in consideration of (4.11) and (4.12) we can write

$$
\begin{aligned}
\frac{d}{d t} \iint \mathfrak{W}_{n}\{\psi\} d F & -\iint \mathfrak{W}_{n}\left\{\frac{d \psi}{d t}\right\} d F \\
& =\iint\{\psi\}[\nabla p, \nabla \sigma]_{n} d F
\end{aligned}
$$

Equation (4.14) represents the integral form of the new vorticity theorem for a field function $\psi$ and discontinuous derivative $d \psi / d t$ on the surface $F$.
If, on the surface $F$, only the field function $\psi$ becomes discontinuous;

$$
\{\psi\}=\psi_{+0}-\psi_{-0} \neq 0,
$$

while $d \psi / d t$, however, remains continuous:

$$
\left\{\frac{d \psi}{d t}\right\}=\left(\frac{d \psi}{d t}\right)_{+0}-\left(\frac{d \psi}{d t}\right)_{-0}=0,
$$

then (4.14) reduces to

$$
\frac{d}{d t} \iint\{\psi\} \mathfrak{W}_{n} d F=\iint\{\psi\}[\nabla p, \nabla \sigma]_{n} d F .
$$

By properly specifying the function $\psi$, Bjerknes' circulation theorem results from equation (4.15).

\section{The circulation theorem of $\mathrm{V}$. Bjerknes as a special case of the new vorticity theorem}

The surface $\Sigma$, which contains the surface $F$, divides the fluid into an "upper fluid half-space" $R_{1}$ and a "lower fluid half-space" $R_{2}$. The surface $\Sigma$, and with it $F$, moves with the current, such that particles which are in $R_{1}$ always remain in $R_{1}$ and particles in $R_{2}$ always remain in $R_{2}$, while the common boundary $\Sigma$ (with the surface $F$ ) of the two fluid spaces $R_{1}$ and $R_{2}$ deforms itself according to the fbw. We now imagine that each fluid particle in $R_{1}$ is assigned a same constant number $Z_{1}$; thus, this number identifies each individual fluid element belonging to the fluid half-space $R_{1}$. Similarly, we imagine all fliid particles from $R_{2}$ possessing the same constant number $Z_{2}$. The numbers $Z_{1}$ and $Z_{2}$ do not characterize the fluid particles individually, because, for instance, all fluid particles in $R_{1}$ are assigned the same number $Z_{1}$; rather, the numbers $Z_{1}$ and $Z_{2}$ are group distinguisher indices that decide which of the two applicable groups of fluid particles each particle belongs to. However, each fluid element maintains the assigned number of $Z_{1}$ or $Z_{2}$ individually:

$$
\frac{d}{d t} Z_{1}=\frac{d}{d t} Z_{2}=0
$$

For example, a particle initially assigned a number $Z_{1}$ and belonging to the fluid half-space $R_{1}$ cannot belong to $R_{2}$ later and not have the assigned number $Z_{2}$ with it, since the particle would have to cross the surface $\Sigma$. This is impossible since the same number is always associated with the same particle, if $\Sigma$, as adopted, moves along with the fbw. However, the number (either $Z_{1}$ or $Z_{2}$ ) changes discontinuously at the surfaces $\Sigma$ and $F$ as $\left|Z_{1}-Z_{2}\right| \neq 0$. The restriction of a fluid element to either the fluid half-space $R_{1}$ or to the fluid half-space $R_{2}$ with these two characteristic numbers $Z_{1}$ 


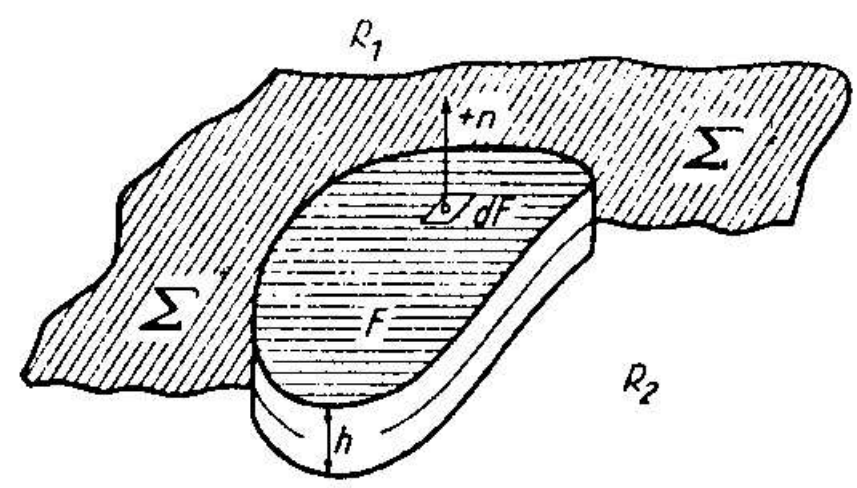

Fig. 4.1

and $Z_{2}$ thus forms a discontinuous $\psi$-field with the characteristics

$$
\begin{array}{lll}
\psi=Z_{1}, & \frac{d \psi}{d t}=0 & \left(\text { in } R_{1}\right) \\
\psi=Z_{2}, & \frac{d \psi}{d t}=0 & \left(\text { in } R_{2}\right)
\end{array}
$$

as well as

$$
\begin{aligned}
\{\psi\} & =\psi_{+0}-\psi_{-0} \\
& =Z_{1}-Z_{2} \neq 0 \quad(\text { on } \Sigma \text { and } / \text { or } F)
\end{aligned}
$$

and

$$
\begin{aligned}
\left\{\frac{d \psi}{d t}\right\} & =\left(\frac{d \psi}{d t}\right)_{+0}-\left(\frac{d \psi}{d t}\right)_{-0} \\
& =0, \quad(\text { on } \Sigma \text { and/or } F)
\end{aligned}
$$

where for this $\psi$-function the new vorticity theorem of the form (4.15) applies. Since $\{\psi\}=Z_{1}-Z_{2}$ is a constant jump value on $\Sigma$ and/or $F$, the equation (4.15) immediately results in

$$
\begin{aligned}
\left(Z_{1}-Z_{2}\right) \frac{d}{d t} \iint & \mathfrak{W}_{n} d F \\
& =\left(Z_{1}-Z_{2}\right) \iint[\nabla p, \nabla \sigma]_{n} d F,
\end{aligned}
$$

or through division by $Z_{1}-Z_{2} \neq 0$,

$$
\frac{d}{d t} \iint \mathfrak{W}_{n} d F=\iint[\nabla p, \nabla \sigma]_{n} d F,
$$

which is the equation that represents Bjerknes' circulation theorem.

We note that

$$
\iint[\nabla p, \nabla \sigma]_{n} d F=N(p, \sigma)
$$

is the quantity of isobar-isostere unit solenoids in $F$ and

$$
\begin{aligned}
\iint \mathfrak{W}_{n} d F & =\iint\left(\operatorname{rot}_{n} \mathbf{v}+2 \mathbf{f}_{n}\right) d F \\
& =\oint v_{s} d s+2 \omega S
\end{aligned}
$$

is the absolute circulation along the boundary of $F$. Then, through Stokes' theorem,

$$
\iint \operatorname{rot}_{n} \mathbf{v} d F=\oint v_{s} d s=C
$$

is the circulation $C$ relative to the Earth (rotating system). Furthermore,

$$
\begin{aligned}
2 \iint \mathbf{f}_{n} d F & =2 \iint \omega \cos (n, \mathbf{f}) d F \\
& =2 \omega S
\end{aligned}
$$

is twice the angular velocity $\omega=|\mathbf{f}|$ multiplied by the projection $S=\iint \cos (n, \mathbf{f}) d F$ of the area $F$ onto any plane (e.g., equatorial plane) perpendicular to the rotation axis, and thus (4.18) is equivalent with the usual version of the Bjerknes' circulation theorem:

$$
\frac{d C}{d t}+2 \omega \frac{d S}{d t}=N(p, \sigma)
$$

Following (4.15) one obtains Lord Kelvin's circulation theorem for irrotational coordinates $(\mathbf{f}=0)$ and a barotropic fluid $(p=p(\sigma))$ [W. THOMSON (1869)]:

$$
\frac{d}{d t} \oint v_{s} d s=0
$$

In summary, from the new hydrodynamic vorticity theorem in its differential and integral forms, all wellknown vorticity and circulation equations, respectively, can be derived as special cases. In addition, the new vorticity theorem results in still unknown special cases, for example, a conservation law valid for polytropic fbws [3] ( $\psi=$ polytropic temperature), for which $\mathbf{F}$. MORÀN [4] has furnished a very appealing proof.

\section{References}

1] H. Ertel, Met. Z. 1942.

2] H. Ertel, Physik. Z. 1942.

3] H. Ertel, Die Naturwissenschaften 1942.

4] F. Moràn, Revista de Geofi sica 1942. 


\section{ERTEL (1952): On the physical significance of functions arising in the Clebsch transformation of the hydrodynamical equations}

\section{Geometry and kinematics of a stationary barotropic fluid}

In the Clebsch transformation of the hydrodynamic equations [Ref. $(1,2,3)]$ the wind velocity vector $\mathbf{v}$ is represented by the superposition of two vectors [Ref. (4)] involving the three scalar functions $\varphi, \lambda, \mu$ :

$$
\mathbf{v}=\nabla \varphi+\lambda \nabla \mu
$$

from which it follows that the functions $\lambda$ and $\mu$ are related to the vorticity vector $\boldsymbol{\xi}=\nabla \times \mathbf{v}$ in accordance with

$$
\boldsymbol{\xi}=\nabla \lambda \times \nabla \mu .
$$

Thus, at each point in the fbw the direction of the vorticity vector is along the intersection of the two surfaces $\lambda=$ const and $\mu=$ const.

At the same time, this intersection curve itself crosses the energy surface

$$
H=\frac{v^{2}}{2}+\Phi+\int \frac{d p}{\rho}=\text { const },
$$

which contains the regarded point if, as is always assumed in the following, we consider the stationary flow of an ideal barotropic fluid. In the expression (5.3) for the total energy $H$ per unit mass, we define $v=|\mathbf{v}|$ and $\Phi$ as the potential of external forces. For all flid particles, the presupposed barotropy is ensured by the existence of a single piezotropic relation $\rho=\rho(p)(\rho=$ density, $p=$ pressure). The sum $\Phi+\int d p / \rho$ represents the total potential energy per unit mass.

In Eulerian form fluid movement is described by the equation

$$
\frac{D \mathbf{v}}{D t}=-\nabla\left(\Phi+\int \frac{d p}{\rho}\right)
$$

with the material derivative operator

$$
\mathbf{v} \cdot \nabla=\frac{D}{D t},
$$

or by the equation

$$
\frac{D \mathbf{v}}{D t}=(\mathbf{v} \cdot \nabla) \mathbf{v}=\nabla\left(\frac{v^{2}}{2}\right)-\mathbf{v} \times \nabla \times \mathbf{v},
$$

which is equivalent to (5.4) because

$$
\mathbf{v} \times \boldsymbol{\xi}=\nabla H .
$$

Scalar multiplication of (5.7) with (5.2) results in $(x, y, z$ are orthogonal cartesian coordinates):

$$
\boldsymbol{\xi} \cdot \nabla H=\nabla H \cdot(\nabla \lambda \times \nabla \mu)=\frac{\partial(H, \lambda, \mu)}{\partial(x, y, z)}=0,
$$

an equation which shows the geometric collapse of the surface system $\lambda=$ const, $\mu=$ const, $H=$ const, and which leads analytically to the existence of a functional relationship

$$
H=H(\lambda, \mu) .
$$

Scalar multiplication of the momentum equation (5.7) with the wind velocity vector $\mathbf{v}$ yields

$$
\mathbf{v} \cdot \nabla H=\frac{D H}{D t}=0,
$$

which represents the conservation law for the total energy and which expresses in a geometric sense a coincidence of the streamlines with the energy surface. Vorticity lines (with tangents $\boldsymbol{\xi}$ ) and streamlines (with tangents $\mathbf{v}$ ) form a grid on each energy surface, whereby the angle of intersection (the angle between $\mathbf{v}$ and $\boldsymbol{\xi}$ ) is determined by the equation

$$
|\mathbf{v}| \cdot|\boldsymbol{\xi}| \cdot \sin (\mathbf{v}, \boldsymbol{\xi})=\frac{\partial H}{\partial n},
$$

which follows from (5.7), with $n$ denoting the positive normal (pointing to larger energy values) to the energy surface. If $\delta n$ denotes the distance between two neighboring energy surfaces of constant energy difference $\left(\frac{\partial H}{\partial n} \delta n=\right.$ const $)$, the equation

$$
\delta n \cdot|\mathbf{v}| \cdot|\boldsymbol{\xi}| \cdot \sin (\mathbf{v}, \boldsymbol{\xi})=\text { const },
$$

which follows from (5.11), can characterize the fbw in terms of the streamline/vorticity grid on the energy surface [Ref. $(5,6,7)]$. By the introduction of unit volumes, formed from energy surfaces and the streamline/vorticity grid, these views can be formulated in an even simpler way [Ref. (8)].

The substitution of (5.2) and (5.9) into (5.7) results in the relation

$$
\mathbf{v} \times(\nabla \lambda \times \nabla \mu)=\frac{\partial H}{\partial \lambda} \nabla \lambda+\frac{\partial H}{\partial \mu} \nabla \mu,
$$

or equivalently,

$$
(\mathbf{v} \cdot \nabla \mu) \nabla \lambda-(\mathbf{v} \cdot \nabla \lambda) \nabla \mu=\frac{\partial H}{\partial \lambda} \nabla \lambda+\frac{\partial H}{\partial \mu} \nabla \mu,
$$


and the existence of Hamilton's canonical equations

$$
\frac{D \mu}{D t}=\frac{\partial H}{\partial \lambda}, \quad \frac{D \lambda}{D t}=-\frac{\partial H}{\partial \mu}
$$

follows [Ref. (9)] for the functions $(\lambda, \mu)$. The above connection of the functions $\lambda, \mu$ with the energy function $H$ now permits the conclusion that the functions $\lambda, \mu$ must possess a physical interpretation that goes beyond their geometric-kinematic meaning (as determinants of the vorticity lines); this conclusion can also be expanded to the function $\varphi$ in equation (5.1).

\section{Transition to the Lagrangian form of hydrody- namics}

For the retrieval of the physical meaning of the functions $\varphi, \lambda, \mu$ it is advisable to use a transformation of the Eulerian equations (5.4) to the Lagrangian equations

$$
\frac{\partial^{2} x_{j}}{\partial t^{2}} \frac{\partial x_{j}}{\partial a_{i}}=-\frac{\partial}{\partial a_{i}}\left(\Phi+\int \frac{d p}{\rho}\right) \quad(i, j=1,2,3) .
$$

Here,

$$
x_{j}=x_{j}\left(a_{1}, a_{2}, a_{3}, t\right) \quad(j=1,2,3)
$$

are the cartesian position coordinates at time $t$ and $a_{1}, a_{2}, a_{3}$ (labeling coordinates) are the position coordinates at time $t=0$, which in a broader sense characterize the motion of individual fuid particles. In (5.16) the Einstein summation convention has been used, so that if an index appears twice, it is summed from 1 to 3. Together with the continuity equation

$$
\rho \frac{\partial\left(x_{1}, x_{2}, x_{3}\right)}{\partial\left(a_{1}, a_{2}, a_{3}\right)}=\rho_{0}=\rho\left(a_{1}, a_{2}, a_{3}, 0\right)
$$

and the piezotropic relation

$$
\rho=\rho(p),
$$

equations $(5.16,5.18,5.19)$ form a system of five equations for the three functions (5.17), the pressure $p$ and the density $\rho$.

Now let

$$
F\left(\stackrel{*}{x}_{1}, \stackrel{*}{x}_{2}, \stackrel{*}{x}_{3}\right)=0
$$

be the equation of a surface lying in the space, related to the same orthogonal cartesian system $x_{i}(i=1,2,3)$, which serves for the determination of the position coordinates (5.17); the variables with attached stars denote those coordinates which satisfy the surface equation (5.20). The surface may possess a continuously variable tangent plane and be oriented in such a manner that it is punctured by the streamlines, but the exact alignment of the surface relative to the streamline system is as yet unspecified.

The position coordinates (5.17) of a particle with initial coordinates $a_{1}, a_{2}, a_{3}$ must, at the time $\vartheta$ when this particle lies on the surface $F\left(\stackrel{*}{x}_{1}, \stackrel{*}{x_{2}}, \stackrel{*}{x_{3}}\right)=0$, agree with the coordinates $\stackrel{*}{x}_{1}, \stackrel{*}{x_{2}}, \stackrel{*}{x_{3}}$, so that:

$$
\stackrel{*}{x}_{j}=x_{j}\left(a_{1}, a_{2}, a_{3}, \vartheta\right) \quad(j=1,2,3)
$$

and the equation of the surface becomes

$$
\begin{aligned}
& F\left(x_{1}\left(a_{1}, a_{2}, a_{3}, \vartheta\right),\right. \\
& \quad x_{2}\left(a_{1}, a_{2}, a_{3}, \vartheta\right), \\
& \left.\quad x_{3}\left(a_{1}, a_{2}, a_{3}, \vartheta\right)\right)=0 .
\end{aligned}
$$

The solution of this equation for $\vartheta$ yields:

$$
\vartheta=\vartheta\left(a_{1}, a_{2}, a_{3}\right),
$$

which indicates the time at which an individually labeled particle $a_{1}, a_{2}, a_{3}$ lies in the given surface $F=0$.

For the following argument it is useful to define the wind components

$$
v_{j}=\frac{\partial x_{j}}{\partial t} \quad(j=1,2,3),
$$

the kinetic energy (per unit mass)

$$
\frac{1}{2}\left(\frac{\partial x_{j}}{\partial t}\right)^{2}=\frac{1}{2} v_{j} v_{j}=\frac{1}{2} v^{2},
$$

and the Lagrangian function

$$
L=\frac{1}{2} v^{2}-\left(\Phi+\int \frac{d p}{\rho}\right),
$$

and thereby rewrite the Lagrangian equations (5.16) in the following form:

$$
\frac{\partial}{\partial t}\left(v_{j} \frac{\partial x_{j}}{\partial a_{i}}\right)=\frac{\partial L}{\partial a_{i}} .
$$

\section{The physical meaning of the functions $\varphi, \lambda, \mu$}

We integrate equations (5.27) from $t=$ $\vartheta\left(a_{1}, a_{2}, a_{3}\right)$ to time $t>\vartheta$ and obtain

$$
v_{j} \frac{\partial x_{j}}{\partial a_{i}}=\left(v_{j}\right)_{\vartheta}\left(\frac{\partial x_{j}}{\partial a_{i}}\right)_{\vartheta}+\int_{\vartheta}^{t} \frac{\partial L}{\partial a_{i}} d t .
$$

Through differentiation with respect to $a_{i}$ it follows from (5.21) that

$$
\frac{\partial x_{j}^{*}}{\partial a_{i}}=\left(\frac{\partial x_{j}}{\partial a_{i}}\right)+\left(v_{j}\right)_{\vartheta} \frac{\partial \vartheta}{\partial a_{i}},
$$


and therefore (5.28) yields:

$$
\begin{aligned}
v_{j} \frac{\partial x_{j}}{\partial a_{i}} & =\left(v_{j}\right)_{\vartheta} \frac{\partial x_{j}^{*}}{\partial a_{i}} \\
& +\frac{\partial}{\partial a_{i}} \int_{\vartheta}^{t} L d t+\left\{(L)_{\vartheta}-\left(v_{j}\right)_{\vartheta}^{2}\right\} \frac{\partial \vartheta}{\partial a_{i}},
\end{aligned}
$$

where we have used the formula

$$
\frac{\partial}{\partial a_{i}} \int_{\vartheta}^{t} L d t=\int_{\vartheta}^{t} \frac{\partial L}{\partial a_{i}} d t-(L)_{\vartheta} \frac{\partial \vartheta}{\partial a_{i}} .
$$

Now, in (5.30)

$$
\int_{\vartheta}^{t} L d t=W
$$

represents the Hamiltonian action function [Ref. (10, 11,12 )], and furthermore (with $v_{j}^{2}=v_{j} v_{j}=v^{2}$ )

$$
\begin{aligned}
(L)_{\vartheta}-\left(v_{j}\right)_{\vartheta}^{2} & =-\left\{\frac{v^{2}}{2}+\left(\Phi+\int \frac{d p}{\rho}\right)\right\} \\
& =-H_{\vartheta}=-H
\end{aligned}
$$

is the negative of the total energy (per unit mass) of the observed particle at time $\vartheta$, which equals $\left(H_{\vartheta}=H\right)$, because of the conservation law (5.10), the negative total energy $-H$ for $t \geq \vartheta$, so that (5.30) takes the form

$$
v_{j} \frac{\partial x_{j}}{\partial a_{i}}=\left(v_{j}\right)_{\vartheta} \frac{\partial *_{j}^{*}}{\partial a_{i}}+\frac{\partial W}{\partial a_{i}}-H \frac{\partial \vartheta}{\partial a_{i}} .
$$

Equation (5.34) is the result of the integration of the Lagrangian momentum equations (5.16), or equivalently (5.27), over the time interval of the movement of a particle along a certain streamline segment. We now consider a closely-adjoining streamline and on this streamline a particle that at time $t=0$ has the associated labeling-coordinate $a_{i}+\delta a_{i}(i=1,2,3)$. With

$$
\frac{\partial x_{j}}{\partial a_{i}} \delta a_{i}=\delta x_{j}, \quad \frac{\partial x_{j}^{*}}{\partial a_{i}} \delta a_{i}=\delta \stackrel{x}{x}_{j}
$$

and

$$
\frac{\partial W}{\partial a_{i}} \delta a_{i}=\delta W, \quad \frac{\partial \vartheta}{\partial a_{i}} \delta a_{i}=\delta \vartheta
$$

it follows from (5.34) that

$$
v_{j} \delta x_{j}=\left(v_{j}\right)_{\vartheta} \delta \stackrel{x}{x}_{j}+\delta W-H \delta \vartheta
$$

from which we can write

$$
v_{j} \delta x_{j}=\left(v_{j}\right)_{\vartheta} \delta^{*} x_{j}+\delta W+H \delta(t-\vartheta),
$$

since the end time $t$ is not varied. Now $\delta x_{j}^{*}(j=$ $1,2,3)$ denotes the infinitesimal vector that extents from the intersection point $P\left(\stackrel{*}{x}_{1}, \stackrel{*}{x_{2}}, \stackrel{*}{x_{3}}\right)$ of the first streamline with the surface $F=0$ to the intersection point $P\left(\stackrel{*}{x}_{1}+\delta \stackrel{*}{x}_{1}, \stackrel{*}{x}_{2}+\delta \stackrel{*}{x}_{2}, \stackrel{*}{x}_{3}+\delta \stackrel{*}{x}_{3}\right)$ of the second streamline with the surface $F=0$. We now specify the situation, which was not yet defined in section II, concerning the orientation of the surface $F=0$ relative to the streamline system, by demanding that the surface $F=0$ should cut the streamline system orthogonally. Then

$$
\left(v_{j}\right)_{\vartheta} \delta^{*} x_{j}=0
$$

and equation (5.38) reduces to

$$
v_{j} \delta x_{j}=\delta W+H \delta \Theta,
$$

where

$$
\Theta=t-\vartheta\left(a_{1}, a_{2}, a_{3}\right)
$$

denotes the running time of the particle $\left(a_{1}, a_{2}, a_{3}\right)$ from the time $\vartheta\left(a_{1}, a_{2}, a_{3}\right)$ of its intersection with the orthogonal surface $F=0$ to the time $t$.

We now go back to the Eulerian form of hydrodynamics and select the cartesian coordinates $x_{j}(j=$ $1,2,3)$ as arguments, so that from (5.40) we obtain

$$
v_{j} \delta x_{j}=\left(\frac{\partial W}{\partial x_{j}}+H \frac{\partial \Theta}{\partial x_{j}}\right) \delta x_{j},
$$

and then because of the free choice of $\delta a_{i}$ or $\delta x_{j}$ it follows that

$$
v_{j}=\frac{\partial W}{\partial x_{j}}+H \frac{\partial \Theta}{\partial x_{j}},
$$

or in vector form

$$
\mathbf{v}=\nabla W+H \nabla \Theta
$$

Hence, the task is solved, since a comparison with (5.1) results in:

$$
\begin{aligned}
\varphi=W & =\int_{\vartheta}^{t} L d t \\
& =\int_{\vartheta}^{t}\left\{\frac{v^{2}}{2}-\left(\Phi+\int \frac{d p}{\rho}\right)\right\} d t \\
& =\text { Hamilton's action function, } \\
\lambda=H & =\frac{v^{2}}{2}+\left(\Phi+\int \frac{d p}{\rho}\right) \\
& =\text { total energy (per unit mass), } \\
& \begin{array}{r}
\mu=\Theta=t-\vartheta\left(a_{1}, a_{2}, a_{3}\right) \\
=\text { running time },
\end{array}
\end{aligned}
$$


with the latter measured from the time $\vartheta\left(a_{1}, a_{2}, a_{3}\right)$ of the intersection of the particle $\left(a_{1}, a_{2}, a_{3}\right)$ with the surface that cuts the streamline system orthogonally. Which of the infinitely many orthogonal surfaces is selected as the "zero-surface" for measuring the running time $\Theta$ is unimportant for the computation of the stationary velocity field from equation (5.44), if in the area between two such orthogonal surfaces the hydrodynamic momentum equations (5.4), or equivalently (5.7), apply. It should be noted that the Hamiltonian function $W$ in (5.44) is to be formed for the running time interval $t-\vartheta=\Theta$.

I will discuss elsewhere the modified interpretation of $\varphi, \lambda, \mu$ of the representation (5.44) which occurs in the case of non-stationary fbws.

Scalar multiplication of equation (5.44) with the velocity vector $\mathbf{v}$ results in the identity $v^{2}=v^{2}$, because, first of all, in consideration of (5.5):

$$
v^{2}=\frac{D W}{D t}+H \frac{D \Theta}{D t}
$$

and furthermore, according to (5.32) and (5.41):

$$
\frac{D W}{D t}=L=\frac{v^{2}}{2}-\left(\Phi+\int \frac{d p}{\rho}\right), \quad \frac{D \Theta}{D t}=1,
$$

and

$$
\frac{D W}{D t}+H \frac{D \Theta}{D t}=L+H=v^{2} .
$$

The Hamiltonian canonical equations (5.15) are identically satisfied, because, with $(5.46,5.47)$ :

$$
\frac{D \mu}{D t}=1, \quad \frac{\partial H}{\partial \lambda}=1,
$$

and from $(5.10,5.46,5.47)$ :

$$
\frac{D \lambda}{D t}=0, \quad \frac{\partial H}{\partial \mu}=0 .
$$

\section{Introduction of the "reduced action function"}

We now introduce in (5.44) the "reduced action function" defined by [Ref. (13)]

$$
\begin{aligned}
S=W+H \Theta & =\int_{\vartheta}^{t}(L+H) d t \\
& =\int_{\vartheta}^{t} v^{2} d t=\int_{B}^{B+\Delta B} v d s,
\end{aligned}
$$

where $d s=v d t$ denotes a line element of the trajectory (also a streamline in the case of stationary motion) and $\Delta B$ is the length of this trajectory from the intersection point with the orthogonal surface $F=0$ up to the point $\left(x_{1}, x_{2}, x_{3}\right)$. Thus, from (5.44), one now obtains the representation

$$
\mathbf{v}=\nabla S-\Theta \nabla H
$$

or written in more detail:

$$
\mathbf{v}=\nabla\left(\int_{B}^{B+\Delta B} v d s\right)-\left(\int_{B}^{B+\Delta B} \frac{d s}{v}\right) \nabla H,
$$

where the running time (the elapsed time ) is given by

$$
\Theta=\int_{B}^{B+\Delta B} \frac{d s}{v}
$$

( $B=$ the length of the trajectory from $a_{i}$ to ${ }_{i}^{*} ; i=$ $1,2,3$.)

\section{Application to asynchronous-periodical flows}

In a stationary asynchronous-periodical fbw field, the fluid particles pass through closed streamlines (trajectories, particle tracks) with circulation times $\tau$ or frequencies $\nu=1 / \tau$ that differ for trajectories on different energy surfaces. Now the velocity vector $\mathbf{v}$ for a particle on a closed orbit $C$ of the length $K=\int_{C} d s$ changes periodically after the circulation time, so from $(5.54,5.55)$ we have $\left(B_{1}=B+\Delta B\right)$ :

$$
\begin{aligned}
\mathbf{v} & =\nabla\left(\int_{B}^{B_{1}} v d s\right)-\Theta \nabla H \\
& =\nabla\left(\int_{B}^{B_{1}+K} v d s\right)-(\Theta+\tau) \nabla H,
\end{aligned}
$$

and also

$$
0=\nabla\left(\int_{B_{1}}^{B_{1}+K} v d s\right)-\tau \nabla H
$$

Because

$$
\int_{B_{1}}^{B_{1}+K} v d s=\oint v d s=\Gamma
$$

is the circulation along the closed path, one finds, after scalar multiplication of equation (5.58) with a differential vector $d \mathbf{r}=\left(d x_{1}, d x_{2}, d x_{3}\right)$, that the changes $d H$ and $d \Gamma$ from an arbitrary point on an orbit lying in the energy surface $H$ with the circulation $\Gamma$ to a point on the energy surface $H+d H=H+d \mathbf{r} \cdot \nabla H$ with the circulation $\Gamma+d \Gamma=\Gamma+d \mathbf{r} \cdot \nabla \Gamma$ leads, are related by

$$
\tau=\frac{d \Gamma}{d H}
$$


which represents the hydrodynamic generalization of a result from the particle dynamics of single periodic motions in relationship with the Hamilton-Jacobi theory [Ref. (14)].

\section{Relation to the hydrodynamical conservation principle of $\mathrm{H}$. Ertel and C.-G. Rossby}

One can define

$$
\hat{W}=\int_{0}^{t} L d t
$$

as the action function with lower integration limit $t=0$. Then, even for non-stationary fbws, the Ertel-Rossby [Ref. $(15,16]$ conservation law

$$
\frac{d}{d t}\{\sigma \boldsymbol{\xi} \cdot(\mathbf{v}-\nabla \hat{W})\}=0,
$$

holds ( $\sigma=1 / \rho=$ specific volume) with the Eulerian material derivative operator

$$
\frac{d}{d t}=\frac{\partial}{\partial t}+\mathbf{v} \cdot \nabla
$$

For stationary fbw fields, $d / d t$ must be replaced with the operator $\mathbf{v} \cdot \nabla=D / D t$, so that

$$
\frac{D}{D t}\{\sigma \boldsymbol{\xi} \cdot(\mathbf{v}-\nabla \hat{W})\}=0
$$

a result which can be easily deduced from (5.44). To see this, we form, out of (5.44) and in accordance with (5.41), the corresponding equations

$$
\mathbf{v}=\nabla W-H \nabla \vartheta
$$

and

$$
\boldsymbol{\xi}=\nabla \times \mathbf{v}=-\nabla H \times \nabla \vartheta .
$$

Furthermore, through the introduction of

$$
\begin{aligned}
\hat{W}=\int_{0}^{t} L d t & =\int_{0}^{\vartheta} L d t+\int_{\vartheta}^{t} L d t \\
& =W^{*}+W,
\end{aligned}
$$

where, in accordance with (5.23),

$$
\stackrel{*}{W}=\int_{0}^{\vartheta} L d t
$$

is independent of the time $t>\vartheta$, we can rewrite (5.65) as

$$
\mathbf{v}-\nabla \hat{W}=-\nabla \stackrel{*}{W}-H \nabla \vartheta
$$

Then scalar multiplication of (5.66) with (5.69) yields first of all:

$$
\begin{aligned}
\boldsymbol{\xi} \cdot(\mathbf{v}-\nabla \hat{W}) & =\nabla \stackrel{*}{W} \cdot(\nabla H \times \nabla \vartheta) \\
& =\frac{\partial(\stackrel{*}{W}, H, \vartheta)}{\partial\left(x_{1}, x_{2}, x_{3}\right)},
\end{aligned}
$$

and further through multiplication with the continuity equation (5.18), with the aid of the multiplication rule for the functional determinants:

$$
\rho_{0} \boldsymbol{\xi} \cdot(\mathbf{v}-\nabla \hat{W})=\rho \frac{\partial(\stackrel{*}{W}, H, \vartheta)}{\partial\left(a_{1}, a_{2}, a_{3}\right)} .
$$

Through introduction of the specific volumes $\left(\sigma, \sigma_{0}\right)$, one obtains the formula

$$
\sigma \boldsymbol{\xi} \cdot(\mathbf{v}-\nabla \hat{W})=\sigma_{0} \frac{\partial(\stackrel{*}{W}, H, \vartheta)}{\partial\left(a_{1}, a_{2}, a_{3}\right)},
$$

in which the functions appearing on the right side are independent of time on each marked particle $\left(a_{1}, a_{2}, a_{3}\right)$, so that the use of the $D / D t$-operator yields the form (5.64) of the conservation relation.

\section{Vortex lines}

From (5.44) it follows that

$$
\boldsymbol{\xi}=\nabla \times \mathbf{v}=\nabla H \times \nabla \Theta,
$$

i.e., the vorticity lines are curves which are formed by the intersections of the running time surfaces $\Theta=$ const with the energy surfaces $H=$ const.

\section{Summary}

The physical meaning of the three scalar functions $(\varphi, \lambda, \mu)$ appearing in the Clebsch-transformation of the hydrodynamic equations is determined by means of the Lagrangian for the case of stationary fbw in a barotropic fluid. The fbw is determined by the functions $\lambda=$ total energy, $\mu=$ elapsed time after the fluid particle penetrates a surface orthogonal to the streamline system, and $\varphi=$ the Hamiltonian action integral, with the integration extended over the running time. The determination of the physical meaning of these functions enables in a more simple manner the derivation of a theorem concerning asynchronous-periodical fbws as well as the proof of the Ertel-Rossby conservation relation of hydrodynamics.

The physical interpretation given here for the three scalar functions appearing in the Clebschtransformation applies to every stationary fbw field for which there exist surfaces orthogonal to the streamline 
system. (Regarding fbw regimes for which such orthogonal surfaces do not exist, see, for example, Prásil [Ref. (17)]). The physical meaning of the functions $\varphi, \lambda, \mu$ pointed out above can be deduced in another way by application of Hamilton's variational principle [Ref. (18)], a topic I will discuss in detail elsewhere in connection with the generalization to the non-stationary case.

It is a pleasure for me to thank my dear friend and colleague Prof. Hilding Köhler of Uppsala, who, in comprehensive discussions over hydrodynamic questions, encouraged me to investigate the present problem.

\section{References}

1) A. Clebsch, Über die Integration der hydrodynamischen Gleichungen. Crelles Journal, Bd. 56, Berlin 1859, S. 1-10.

2) W. WiEn, Lehrbuch der Hydrodynamik. Leipzig 1900, S. 23-25.

3) H. LAMB, Lehrbuch der Hydrodynamik. 2. Aufl. der deutschen Ausgabe. Leipzig u. Berlin 1931, S. 270-272.

4) V. BJerknes und Mitarbeiter, Physikalische Hydrodynamik. Berlin 1933, 146.

5) H. LAMB, a. a. O., S. 266.

6) H. LAmb, Hydrodynamics. Sixth Edition, Cambridge 1932, p. 243 f.

7) A. S. Ramsey, A Treatise on Hydromechanics. Part II. Hydrodynamics. London, 1947, p. 249.

8) H. ERTEL und H. KöHLER, Ein Theorem über die stationäre Wirbelbewegung kompressibler
Flüssigkeiten. ZAMM, Bd. 29, Berlin 1949, S. 109-113.

9) H. LAMB, a. a. O. (Berlin 1931), S. 249; (Cambridge 1932), p. 249.

10) E. Madelung, Die mathematischen Hilfsmittel des Physikers. 4. Aufl. Berlin, Göttingen, Heidelberg 1950, S. 364.

11) G. HAMEL, Theoretische Mechanik. Berlin, Göttingen, Heidelberg 1949, S. 317 ff.

12) TH. PösCHL, Einführung in die Analytische Mechanik. Karlsruhe 1949, S. 123 f.

13) G. Joos, Lehrbuch der Theoretischen Physik. 7. Aufl. Leipzig 1950, S. 111.

14) H. ERTEL, Ein Theorem über asynchron-periodische Wirbelbewegungen kompressibler Flüssigkeiten. Miscellanea Acad. Berolinensia, Bd. I. Berlin 1950, S. 62-68.

15) H. Ertel und C.-G. Rossby, Ein neuer Erhaltungssatz der Hydrodynamik. Sitz.-Ber. Dt. Akad. D. Wiss. Berlin, Math.-naturwiss. Kl., 1949, Nr. 1.

16) H. Ertel and C.-G. Rossby, A new Conservation-Theorem of Hydrodynamics. Geofi scia pura e applicata, Milano, Vol. XIV, 1949, p. 189-193.

17) F. PRÁsIL, Technische Hydrodynamik. 2. Aufl. Berlin 1926, S. $75 \mathrm{ff}$.

18) L. DE BRogLIE, Einführung in die Wellenmechanik. Leipzig 1929, S. 15-17.

\section{ERTEL (1955a): Canonical algorithm for hydrodynamic vorticity equations}

\section{Introduction}

The goal of this study is to obtain a general equation that allows derivation of all known hydrodynamic vorticity theorems by specification of a single vector $\Psi$.

In 1942 H. ERTEL [Ref. 1-3] derived the formula

$$
\frac{d}{d t}(\alpha \boldsymbol{\xi} \cdot \nabla \psi)=\alpha \boldsymbol{\xi} \cdot \nabla\left(\frac{d \psi}{d t}\right)+\alpha N(p, \alpha, \psi),
$$

where

$$
\frac{d}{d t}=\frac{\partial}{\partial t}+\mathbf{v} \cdot \nabla
$$

is the material time derivative, $\mathbf{v}$ the velocity vector, $\boldsymbol{\xi}$ the vorticity vector, $\alpha$ the specific volume (recip- rocal of density), $p$ the pressure, $\psi$ the for specification of the available scalar function, and $N(p, \alpha, \psi)=$ $(\nabla p \times \nabla \alpha) \cdot \nabla \psi$ the number of $p, \alpha, \psi$ unit cells in the unit volume.

Equation (6.1) came close to this goal, as it permitted the then (1942) known vorticity theorems to be deduced through specification of the scalar function $\psi$. For instance, in equation (6.1), defining cartesian coordinates $x, y, z$ for $\psi$ results in the vorticity equation components for compressible, baroclinic fluids. In the case of adiabatic or general polytropic changes of state, if we substitute the potential or polytropic temperatures 
for $\psi$, such that

$$
\psi=\psi(p, \alpha), \quad \frac{d \psi}{d t}=0
$$

is valid, the resulting conservation principle that arises from (6.1) [Ref. 4] is

$$
\frac{d}{d t}(\alpha \boldsymbol{\xi} \cdot \nabla \psi)=0
$$

[compare also with Ref. 5-8].

In 1948 a further conservation principle [H. ERTEL and C.-G. RossbY, Ref. 9, 10] for barotropic fluids was found for all particles with the same piezotropic relation $\alpha(p)$

$$
\frac{d}{d t}\{\alpha \boldsymbol{\xi} \cdot(\mathbf{v}-\nabla W)\}=0,
$$

in which $W=\int_{0}^{t} L d t$ is the forcing function ( $L$ is the Lagrange function); (6.2) cannot be considered a special case of (6.1), and this fact suggests the generalization shown below.

\section{Canonical algorithms for hydrodynamic vorticity equations}

To obtain the general formula (the canonical algorithm), which through specification of a vector $\Psi$ yields all known vorticity equations, we start with the hydrodynamic motion equations

$$
\begin{aligned}
\frac{\partial \mathbf{v}}{\partial t}+\nabla\left(\frac{1}{2} v^{2}\right) & -\mathbf{v} \times(2 \boldsymbol{\Omega}+\nabla \times \mathbf{v}) \\
= & -\nabla \phi-\alpha \nabla p
\end{aligned}
$$

( $\boldsymbol{\Omega}=$ Earth's rotation vector, taken to be constant, $\phi=$ geopotential), which we write through introduction of the absolute velocity

$$
\mathbf{V}=\mathbf{v}+\mathbf{\Omega} \times \mathbf{r}
$$

( $\mathbf{r}=$ position vector from a point on the axis of rotation to the fuid particle) and the absolute vorticity

$$
\boldsymbol{\xi}=\nabla \times \mathbf{V}=\nabla \times \mathbf{v}+2 \boldsymbol{\Omega}
$$

in the form

$$
\frac{\partial \mathbf{V}}{\partial t}+\nabla\left(\frac{1}{2} v^{2}\right)-\mathbf{v} \times \boldsymbol{\xi}=-\nabla \phi-\alpha \nabla p,
$$

from which, through application of the curl operation, follows

$$
\frac{\partial \boldsymbol{\xi}}{\partial t}-\nabla \times(\mathbf{v} \times \boldsymbol{\xi})=\mathbf{N}(p, \alpha)
$$

with the solenoidal vector

$$
\mathbf{N}(p, \alpha)=\nabla p \times \nabla \alpha .
$$

After easy manipulation, scalar multiplication with the vector $\Psi$ gives

$$
\frac{\partial}{\partial t}(\boldsymbol{\xi} \cdot \boldsymbol{\Psi})=\boldsymbol{\xi} \cdot \frac{\partial \boldsymbol{\Psi}}{\partial t}+\boldsymbol{\Psi} \cdot \nabla \times(\mathbf{v} \times \boldsymbol{\xi})+\mathbf{N}(p, \alpha) \cdot \mathbf{\Psi} .
$$

We have

$$
\begin{aligned}
\boldsymbol{\Psi} & \cdot \nabla \times(\mathbf{v} \times \boldsymbol{\xi}) \\
& =(\mathbf{v} \times \boldsymbol{\xi}) \cdot \nabla \times \mathbf{\Psi}-\nabla \cdot\{\boldsymbol{\Psi} \times(\mathbf{v} \times \boldsymbol{\xi})\} \\
& =(\mathbf{v} \times \boldsymbol{\xi}) \cdot \nabla \times \mathbf{\Psi}-\nabla \cdot\{\mathbf{v}(\boldsymbol{\xi} \cdot \boldsymbol{\Psi})-\boldsymbol{\xi}(\mathbf{v} \cdot \mathbf{\Psi})\} \\
& =(\mathbf{v} \times \boldsymbol{\xi}) \cdot \nabla \times \boldsymbol{\Psi}-\nabla \cdot\{\mathbf{v}(\boldsymbol{\xi} \cdot \boldsymbol{\Psi})\}+\boldsymbol{\xi} \cdot \nabla(\mathbf{v} \cdot \boldsymbol{\Psi}) \\
& =\boldsymbol{\xi} \cdot\{\nabla(\mathbf{v} \cdot \boldsymbol{\Psi})-\mathbf{v} \times \nabla \times \mathbf{\Psi}\}-\nabla \cdot\{\mathbf{v}(\boldsymbol{\xi} \cdot \mathbf{\Psi})\},
\end{aligned}
$$

so that (6.8) can be written in the form

$$
\begin{aligned}
\frac{\partial}{\partial t}(\boldsymbol{\xi} \cdot \boldsymbol{\Psi}) & +\nabla \cdot\{\mathbf{v}(\boldsymbol{\xi} \cdot \boldsymbol{\Psi})\} \\
=\boldsymbol{\xi} & \cdot\left\{\frac{\partial \boldsymbol{\Psi}}{\partial t}+\nabla(\mathbf{v} \cdot \boldsymbol{\Psi})-\mathbf{v} \times \nabla \times \boldsymbol{\Psi}\right\} \\
& +\mathbf{N}(p, \alpha) \cdot \mathbf{\Psi} .
\end{aligned}
$$

Since

$$
\frac{\partial \boldsymbol{\Psi}}{\partial t}+\nabla(\mathbf{v} \cdot \mathbf{\Psi})-\mathbf{v} \times(\nabla \times \mathbf{\Psi})=\frac{D \boldsymbol{\Psi}}{D t}
$$

is that differential operation by which the substantial change of the scalar product of the vector $\Psi$ can be shown [Ref. 11-12] to be a "material change," and using a line element $\mathbf{d s}$ which follows the fluid, such that

$$
\frac{d}{d t}(\mathbf{\Psi} \cdot \mathbf{d} \mathbf{s})=\frac{D \boldsymbol{\Psi}}{D t} \cdot \mathbf{d} \mathbf{s},
$$

equation (6.9) can be written as

$$
\begin{aligned}
\frac{\partial}{\partial t}(\boldsymbol{\xi} \cdot \boldsymbol{\Psi}) & +\nabla \cdot\{\mathbf{v}(\boldsymbol{\xi} \cdot \boldsymbol{\Psi})\} \\
& =\boldsymbol{\xi} \cdot \frac{D \boldsymbol{\Psi}}{D t}+\mathbf{N}(p, \alpha) \cdot \boldsymbol{\Psi} .
\end{aligned}
$$

After multiplying this equation by $\alpha$ and using

$$
\nabla \cdot\{\mathbf{v}(\boldsymbol{\xi} \cdot \mathbf{\Psi})\}=\mathbf{v} \cdot \nabla(\boldsymbol{\xi} \cdot \mathbf{\Psi})+(\boldsymbol{\xi} \cdot \mathbf{\Psi}) \nabla \cdot \mathbf{v}
$$

and the continuity equation

$$
\alpha \nabla \cdot \mathbf{v}=\frac{\partial \alpha}{\partial t}+\mathbf{v} \cdot \nabla \alpha=\frac{d \alpha}{d t},
$$

we obtain the sought after solution

$$
\frac{d}{d t}(\alpha \boldsymbol{\xi} \cdot \boldsymbol{\Psi})=\alpha \boldsymbol{\xi} \cdot \frac{D \boldsymbol{\Psi}}{D t}+\alpha \mathbf{N}(p, \alpha) \cdot \boldsymbol{\Psi},
$$


where on the right side one must still add the term $\alpha(\nabla \times \mathbf{F}) \cdot \mathbf{\Psi}$ when the external force $\mathbf{F}$ is not deriveable from a potential $\phi$ :

$$
\begin{aligned}
\frac{d}{d t}(\alpha \boldsymbol{\xi} \cdot \boldsymbol{\Psi}) & =\alpha \boldsymbol{\xi} \cdot \frac{D \boldsymbol{\Psi}}{D t} \\
& +\alpha\{\nabla \times \mathbf{F}+\mathbf{N}(p, \alpha)\} \cdot \boldsymbol{\Psi}
\end{aligned}
$$

In the following cases we will always assume there exists a potential of the external force, and thus use the form (6.14) from the established algorithms (6.14, $6.15)$.

\section{Derivation of the hydrodynamic vorticity equa- tion by means of the canonical algorithm by specifi- cation of the vector $\Psi$}

Equations (6.14) and/or (6.15) are to be understood to be general formulae, which supply a corresponding vorticity equation for each special choice of the vector $\Psi$.

A. If, for example, we set $\Psi=\mathbf{i}$ ( $\mathbf{i}=$ the unit vector of the $x$-axis of a cartesian coordinate system), equation (6.14) supplies, since equation (6.10) is

$$
\frac{D \mathbf{i}}{D t}=\nabla v_{x}
$$

the $x$-components of the generalized Helmholtz vorticity equation for compressible and baroclinic fuids

$$
\frac{d}{d t}\left(\alpha \xi_{x}\right)=\alpha \boldsymbol{\xi} \cdot \nabla v_{x}+\alpha N_{x}(p, \alpha) .
$$

One can obtain the $y$ and $z$-components from equation (6.14) by choosing $\boldsymbol{\Psi}=\mathbf{j}$ and $\boldsymbol{\Psi}=\mathrm{k}$ respectively ( $\mathbf{j}, \mathrm{k}$ are unit vectors in the $y$ and $z$ directions). [Compare, for example, Ref. 13].

B. If one sets $\boldsymbol{\Psi}=\nabla \psi(\psi$ is a scalar function) in equation (6.14) in accordance with equation (6.10), we have

$$
\begin{aligned}
\frac{D}{D t} \nabla \psi & =\frac{\partial}{\partial t} \nabla \psi+\nabla(\mathbf{v} \cdot \nabla \psi) \\
& =\nabla\left(\frac{\partial \psi}{\partial t}+\mathbf{v} \cdot \nabla \psi\right)=\nabla\left(\frac{d \psi}{d t}\right)
\end{aligned}
$$

and equation (6.1) will result from equation (6.14).

C. If we set $\mathbf{\Psi}=\mathbf{v}-\nabla W$ in equation (6.14) with the Lagrange function

$$
L=\frac{d W}{d t}=\frac{v^{2}}{2}-\left(\phi+\int \alpha d p\right)
$$

(nonrotating system $\boldsymbol{\Omega}=0$ and autobarotropic $\alpha=$ $\alpha(p)$ ), then equation (6.10) will be

$$
\begin{aligned}
\frac{D}{D t}(\mathbf{v}-\nabla W) & \frac{\partial \mathbf{v}}{\partial t}-\nabla \frac{\partial W}{\partial t}+\nabla\left(v^{2}-\mathbf{v} \cdot \nabla W\right) \\
& -\mathbf{v} \times \nabla \times \mathbf{v} \\
= & \frac{\partial \mathbf{v}}{\partial t}+\nabla v^{2}-\mathbf{v} \times \nabla \times \mathbf{v} \\
& -\nabla\left(\frac{\partial W}{\partial t}+\mathbf{v} \cdot \nabla W\right) \\
= & \frac{\partial \mathbf{v}}{\partial t}+\nabla v^{2}-\mathbf{v} \times \nabla \times \mathbf{v}-\nabla\left(\frac{d W}{d t}\right) \\
= & \frac{\partial \mathbf{v}}{\partial t}+\nabla v^{2}-\mathbf{v} \times \nabla \times \mathbf{v} \\
& -\nabla\left\{\frac{1}{2} v^{2}-\left(\Phi+\int \alpha d p\right)\right\} \\
= & \frac{\partial \mathbf{v}}{\partial t}+\nabla\left(\frac{1}{2} v^{2}\right)-\mathbf{v} \times \nabla \times \mathbf{v} \\
& +\nabla\left(\Phi+\int \alpha d p\right)=0
\end{aligned}
$$

since the hydrodynamic motion equation in this case is

$$
\begin{aligned}
\frac{\partial \mathbf{v}}{\partial t}+\nabla\left(\frac{1}{2} v^{2}\right) & -\mathbf{v} \times \nabla \times \mathbf{v} \\
& =-\nabla\left(\Phi+\int \alpha d p\right)
\end{aligned}
$$

and the conservation principle (6.2) with $\boldsymbol{\xi}=\nabla \times \mathbf{v}$ will result from equation (6.14). In the case of a rotating system, in order to derive the conservation principle (6.2), in which then $\boldsymbol{\xi}$ represents the absolute vorticity $2 \boldsymbol{\Omega}+\nabla \times \mathbf{v}$, and $\mathbf{v}$ is replaced by the absolute velocity,

$$
\mathbf{V}=\mathbf{v}+\boldsymbol{\Omega} \times \mathbf{r}
$$

and the Lagrange function [Ref. 14] is

$$
\begin{aligned}
\stackrel{*}{L}=\frac{d \stackrel{*}{W}}{d t} & =\frac{1}{2} v^{2}+\boldsymbol{\Omega} \cdot(\mathbf{r} \times \mathbf{v}) \\
& =-\left(\Phi+\int \alpha d p\right)
\end{aligned}
$$

in equation (6.14) one must now use $\mathbf{\Psi}=\mathbf{V}-\nabla \stackrel{*}{W}$. Then according to (6.10)

$$
\frac{D}{D t}(\mathbf{V}-\nabla \stackrel{*}{W})=0
$$

based on the hydrodynamic motion equation (6.3) and the conservation principle $(6.2)$ [Ref. 9, 10] we have

$$
\frac{d}{d t}\{\alpha \boldsymbol{\xi} \cdot(\mathbf{V}-\nabla \stackrel{*}{W})\}=0
$$


with $\boldsymbol{\xi}=\nabla \times \mathbf{V}$ (absolute vorticity).

\section{Summary}

We have obtained a general formula (canonical algorithm) that permits derivation of all presently known hydrodynamic vorticity equations in differential form through specification of a single vector.

\section{References}

[1] H. ERTEL, Ein neuer hydrodynamischer Wirbelsatz. Meteorol. Zeitschr. (Braunschweig) 1942, 277-281.

[2] H. ERTEL, Über das Verhältnis des neuen hydrodynamischen Wirbelsatzes zum Zirkulationssatz von V. BJERKNES. Ebenda, 385-387.

[3] H. ERTEL, Über hydrodynamische Wirbelsätze. Physikal. Zeischr. 1942, 526-529.

[4] H. ERTEL, Ein neuer hydrodynamischer Erhaltungassatz. Die Naturwissenschaften (Berlin) 1942, 543-544.

[5] E. KLEInsChmidT jr., Über Aufbau und Entestehung von Zyklonen. Meteorol. Rundschau 1950, 1-6, 54-61; 1951, 89-96.

[6] F. BAuR, Linke's Meteorol. Taschenbuch. Neue Ausgabe. H.Bd. Leipzig 1953, 295-298 (P. RAETHJEN).
[7] K. Oswatitsch, Gasdynamik. Wien 1952, 156.

[8] N.J. Kotschin, I.A. Kibel und N.W. Rose, Theoretische Hydromechanik. Bd. I. Berlin 1954, 501 (Deutsche Ausgabe, red. von K. KRIENES).

[9] H. ERTEL und C.-G. Rossby, A new conservation-theorem of hydrodynamics. Geosfi sica pura e applicata. Milano 1948, Vol. XIV, Fasc. 3-4.

[10] H. ERTel und C.-G. Rossby, Ein neuer Erhaltungs-Satz der Hydrodynamik. Sitz.-Ber. Dt. Akad. d. Wiss. Berlin, Kl. f. Math. u. allgem. Naturwiss., 1949, Nr.1.

[11] W.v. Ignatowsky, Die Vektoranalysis und ihre Anwendungen in der theoretischen Physik. Bd. 11. Leipzig-Berlin 1910, 62.

[12] E. LOHR, Vektor- und Dyadenrechnung für Physiker und Techniker. 2. Aufl. Berlin 1950, 315-317.

[13] H. LAmb, Hydrodynamics. First American Edition New York 1945, 205.

[14] H. ERTEL, Methoden und Probleme der Dynamischen Meteorologie. Berlin 1938 (Ann Arbor, Mich. 1943), 41-44.

\section{ERTEL (1955b): A new hydrodynamical vorticity theorem}

\section{Introduction}

If the motion of a compressible fluid relative to the rotating earth is written through the hydrodynamic vector equation

$$
\begin{aligned}
\frac{\partial \mathbf{v}}{\partial t}+\nabla\left(\frac{1}{2} v^{2}\right) & -\mathbf{v} \times(2 \boldsymbol{\Omega}+\nabla \times \mathbf{v}) \\
& =-\nabla \Phi-\alpha \nabla p
\end{aligned}
$$

where $\mathbf{v}$ is the velocity vector relative to the Earth, $v$ the absolute value of the velocity vector, $\Omega$ the constant rotation vector of the Earth, $\Phi$ the geopotential, $\alpha$ the specific volume (reciprocal of density $\rho$ ), and $p$ the pressure, then the time change of circulation relative to the Earth

$$
\frac{D}{D t} \oint v_{s} d s=\frac{D}{D t} \iint(\nabla \times \mathbf{v})_{n} d F
$$

is a substantial one, moving with the fluid, of the closed curve (a line element of which $=d s$ ) or of the vorticity flux through every material surface (an element of which $=d F, \mathbf{n}=$ positive normal), which has a closed material curve as the boundary; this time change is determined from the circulation equation of $\mathrm{V}$. Bjerknes [Ref. (1-3)] through the equation

$$
\frac{D}{D t}\left(\oint v_{s} d s+2 \Omega F_{\Omega}\right)=N(p, \alpha)
$$

in which

$$
F_{\Omega}=\iint \cos (\mathbf{n}, \boldsymbol{\Omega}) d F
$$

represents the projection of the surface $\iint d F$ onto any latitudinal plane (e.g., the equatorial plane) and where

$$
N(p, \alpha)=\iint(\nabla p \times \nabla \alpha)_{n} d F
$$

represents the number of isobar-isostern unit solenoids in the surface $\iint d F$.

If one introduces the absolute vorticity

$$
\boldsymbol{\xi}=2 \boldsymbol{\Omega}+\nabla \times \mathbf{v}
$$


for which flux through $\iint d F$, the following holds:

$$
\iint \xi_{n} d F=\oint v_{s} d s+2 \Omega F_{\Omega}
$$

then the circulation equation of V. Bjerknes can be written

$$
\frac{D}{D t} \iint \xi_{n} d F=\iint(\nabla p \times \nabla \alpha)_{n} d F .
$$

The operator $D / D t$ which describes the time change of $\iint a_{n} d F$ for a vector a through a moving material surface $\iint d F$ means explicitly the operation

$$
\begin{aligned}
& \frac{D}{D t} \iint a_{n} d F \\
= & \left.\iint\left\{\frac{\partial \mathbf{a}}{\partial t}+\nabla \times(\mathbf{a} \times \mathbf{v})+\mathbf{v} \nabla \cdot \mathbf{a}\right)\right\}_{n} d F,
\end{aligned}
$$

[Ref. (4,5)] which for $\mathbf{a}=\boldsymbol{\xi}$ with $\nabla \cdot \boldsymbol{\xi}=0$, immediately produces the V. Bjerknes circulation equation (7.8) in that the curl of (7.1), with reference to (7.6), results in

$$
\frac{\partial \boldsymbol{\xi}}{\partial t}+\nabla \times(\boldsymbol{\xi} \times \mathbf{v})=\nabla p \times \nabla \alpha .
$$

The resulting vorticity theorem differs from the V. Bjerknes circulation equation in that, in place of the flux of the vorticity vector $\iint \xi_{n} d F$ through a material surface, one looks at the generalized flux $\iint \psi \xi_{n} d F$, where $\psi$ denotes a scalar function $\psi(x, y, z, t)$ of the space coordinates $x, y, z$ and the time $t$.

\section{Derivation of the new vorticity theorem}

We first multiply (7.10) by $\psi$ to obtain

$$
\psi \frac{\partial \boldsymbol{\xi}}{\partial t}+\psi \nabla \times(\boldsymbol{\xi} \times \mathbf{v})=\psi(\nabla p \times \nabla \alpha) .
$$

Because

$$
\psi \nabla \times(\boldsymbol{\xi} \times \mathbf{v})=\nabla \times(\psi \boldsymbol{\xi} \times \mathbf{v})+\nabla \psi \times(\mathbf{v} \times \boldsymbol{\xi})
$$

and

$$
\nabla \psi \times(\mathbf{v} \times \boldsymbol{\xi})=\mathbf{v}(\boldsymbol{\xi} \cdot \nabla \psi)-\boldsymbol{\xi}(\mathbf{v} \cdot \nabla \psi)
$$

we can write

$$
\begin{aligned}
\psi \frac{\partial \boldsymbol{\xi}}{\partial t}+\nabla \times(\psi \boldsymbol{\xi} \times \mathbf{v})+\mathbf{v} & (\boldsymbol{\xi} \cdot \nabla \psi)-\boldsymbol{\xi}(\mathbf{v} \cdot \nabla \psi) \\
= & \psi(\nabla p \times \nabla \alpha) .
\end{aligned}
$$

If we write

$$
\psi \frac{\partial \boldsymbol{\xi}}{\partial t}=\frac{\partial(\psi \boldsymbol{\xi})}{\partial t}-\boldsymbol{\xi} \frac{\partial \psi}{\partial t},
$$

and introduce the Eulerian material derivative operator

$$
\frac{d}{d t}=\frac{\partial}{\partial t}+\mathbf{v} \cdot \nabla
$$

and if we also observe that

$$
\boldsymbol{\xi} \cdot \nabla \psi=\nabla \cdot(\psi \boldsymbol{\xi})
$$

(since $\nabla \cdot \boldsymbol{\xi}=0$ ), the result from (7.14) is the equation

$$
\begin{aligned}
\frac{\partial(\psi \boldsymbol{\xi})}{\partial t}+\nabla \times(\psi \boldsymbol{\xi} \times \mathbf{v})+ & \mathbf{v} \nabla \cdot(\psi \boldsymbol{\xi})-\boldsymbol{\xi} \frac{d \psi}{d t} \\
& =\psi(\nabla p \times \nabla \alpha) .
\end{aligned}
$$

If the subscript $n$ denotes the normal to a surface element of a unbounded material surface $\iint d F$, then from (7.18) it follows that

$$
\begin{gathered}
\iint\left\{\frac{\partial(\psi \boldsymbol{\xi})}{\partial t}+\nabla \times(\psi \boldsymbol{\xi} \times \mathbf{v})+\mathbf{v} \nabla \cdot(\psi \boldsymbol{\xi})\right\}_{n} d F \\
-\iint \xi_{n} \frac{d \psi}{d t} d F=\iint \psi(\nabla p \times \nabla \alpha)_{n} d F,
\end{gathered}
$$

or, with the aid of (7.9),

$$
\begin{aligned}
\frac{D}{D t} \iint \psi \xi_{n} d F & -\iint \xi_{n} \frac{d \psi}{d t} d F \\
& =\iint \psi(\nabla p \times \nabla \alpha)_{n} d F
\end{aligned}
$$

This equation represents the new vorticity theorem, which hereby has been derived from the hydrodynamic vector equation (7.1), with the introduction of the specialized scalar function $\psi$.

\section{Special cases}

From the vorticity theorem (7.20), special cases can be derived by specification of the scalar function $\psi$, so that the open surface $\iint d F$ can be made to be a closed surface.

\section{Examples:}

a) V. Bjerknes' circulation theorem (7.8) can be obtained as a special case of the vorticity theorem (7.20) for

$$
\psi=\text { const. }
$$

b) Let us choose

$$
\psi=T^{-1}\left(\frac{p}{p_{0}}\right)^{\kappa}=\theta^{-1}
$$


( $\theta$ is the potential temperature, $p_{0}$ is the reference pressure, $\left.\gamma=c_{p} / c_{v}, \kappa=(\gamma-1) / \gamma\right)$ and consider adiabatic fbw so that

$$
\frac{d \theta}{d t}=0
$$

From (7.20) we then obtain

$$
\frac{D}{D t} \iint \frac{\xi_{n}}{\theta} d F=\iint \theta^{-1}(\nabla p \times \nabla \alpha)_{n} d F .
$$

The right hand side of this equation can be written as the flux of a solenoidal vector, since it follows from the equation of state for an ideal gas ( $p \alpha=R T, R=c_{p}-c_{v}$ is the gas constant, $T$ is the absolute temperature) that

$$
\theta=\frac{p_{0}^{\kappa}}{R} p^{\frac{1}{\gamma}} \alpha
$$

and therefore that

$$
\theta^{-1}(\nabla p \times \nabla \alpha)=\nabla\left(\frac{p}{p_{0}}\right)^{\kappa} \times \nabla\left(c_{p} \ln \alpha\right) .
$$

Consequently we have

$$
\begin{aligned}
& \frac{D}{D t} \iint \frac{\xi_{n}}{\theta} d F \\
& \quad=\iint\left\{\nabla\left(\frac{p}{p_{0}}\right)^{\kappa} \times \nabla\left(c_{p} \ln \alpha\right)\right\}_{n} d F
\end{aligned}
$$

with the solenoidal vector

$$
\nabla\left(\frac{p}{p_{0}}\right)^{\kappa} \times \nabla\left(c_{p} \ln \alpha\right)=\nabla\left(\frac{1}{\theta}\right) \times \nabla\left(c_{p} T\right) .
$$

Also the choice $\psi=\theta$ (instead of $\psi=\theta^{-1}$ ) gives the fux of a solenoidal vector for the right hand side of (7.20).

c) Generally, the following holds

$$
\frac{D}{D t} \iint J \xi_{n} d F=\iint\{\nabla f(p) \times \nabla g(\alpha)\}_{n} d F
$$

for every hydrodynamic invariant $J(d J / d t=0)$ that allows itself to be represented in the form

$$
J=\frac{d f(p)}{d p} \frac{d g(\alpha)}{d \alpha}
$$

(for example the polytropic temperature during polytropic changes of state).

Suppose one chooses in the vorticity theorem (7.20) a closed surface $S=\iint d s$ for $\iint d F$; this surface encloses the volume $V=\iiint d V$. Since the application of
Gauss' theorem yields

$$
\begin{aligned}
\iint \psi(\nabla p \times \nabla \alpha)_{n} d S & =\iiint(\nabla p \times \nabla \alpha) \cdot \nabla \psi d V \\
& =N(p, \alpha, \psi)
\end{aligned}
$$

the following equation results:

$$
\frac{D}{D t} \iint \psi \xi_{n} d S-\iint \xi_{n} \frac{d \psi}{d t} d S=N(p, \alpha, \psi) .
$$

This is the integral form of the vorticity equation derived by the author in 1942 [Ref. (6)], where $N(p, \alpha, \psi)$ denotes the number of $(p, \alpha, \psi)$ unit cells enclosed in the volume $V$. On the other hand, the vorticity theorem (7.20) can be understood as the generalization of (7.32) for the case of an unbounded surface integration.

\section{Summary}

A hydrodynamic vorticity theorem in a form compatible with the fundamental equation of hydrodynamics for compressible fluids has been derived, from which integral vorticity equations, for example the Bjerknes circulation theorem, can be represented in a simple form through specification of a scalar function.

\section{References}

[1] V. BJERKNES, Über einen hydrodynamischen Zirkulationssatz und seine Anwendung auf die Mechanik der Atmosphäre und des Weltmeeres. Kongl. Svenska Vetenskapsakad. Handl. 31 (1908).

- Das dynamische Prinzip der Zirkulationsbewegungen in der Atmosphäre. Meteorol. Zeitschr. 1900, 97-106. — Zirkulation relativ zu der Erde. Ebenda 1902, 96-108.

[2] V. BJERKNeS und Mitarbeiter, Physikalische Hydrodynamik. Berlin 1933, 146.

[3] J. Holmboe, G. F. Forsythe and W. Gustin, Dynamic Meteorology. New York and London 1945, 295-318.

[4] E. Madelung, Die mathematischen Hilfsmittel des Physikers. 4. Aufl. Berlin-Göttingen-Heidelberg 1950, 203.

[5] R. Gans, Vector Analysis with Applications to Physics. London and Glasgow 1950, 59-60.

[6] H. ERTEL, Über hydrodynamische Wirbelsätze. Physikalische Zeitschrift, 1942, 526-529. 


\section{ERTEL (1956): Orthogonal trajectory systems in stationary two-dimensional fbw of an ideal incompressible fluid}

\section{Remarks on methods for the transformation of the hydrodynamic equations}

A planar fbw field is characterized by the property that the fbw of each and every fluid particle evolves in a plane and the fbw in each and every plane is identical. The entire fbw field is determined by the fbw in one plane, say the $x, y$-plane [M. LAGALLY, L. M. Milne-Thomson, Ref. (1,2)].

In the absence of exterior forces and for stationary motion of an ideal fluid, the Eulerian hydrodynamical equations take the matrix form

$$
\left(\begin{array}{cc}
\frac{\partial v_{x}}{\partial x} & \frac{\partial v_{x}}{\partial y} \\
\frac{\partial v_{y}}{\partial x} & \frac{\partial v_{y}}{\partial y}
\end{array}\right)\left(\begin{array}{c}
v_{x} \\
v_{y}
\end{array}\right)=-\frac{1}{\rho}\left(\begin{array}{c}
\frac{\partial p}{\partial x} \\
\frac{\partial p}{\partial y}
\end{array}\right)
$$

where $v_{x}, v_{y}$ are the wind components, $p$ the pressure, and $\rho$ the density.

The fbw is assumed to be incompressible, so that the trace of the $2 \times 2$ matrix of the velocity derivatives

$$
M=\left(\begin{array}{cc}
M_{x x} & M_{x y} \\
M_{y x} & M_{y y}
\end{array}\right)=\left(\begin{array}{cc}
\frac{\partial v_{x}}{\partial x} & \frac{\partial v_{x}}{\partial y} \\
\frac{\partial v_{y}}{\partial x} & \frac{\partial v_{y}}{\partial y}
\end{array}\right)
$$

vanishes, i.e.,

$$
M_{x x}+M_{y y}=\frac{\partial v_{x}}{\partial x}+\frac{\partial v_{y}}{\partial y}=0
$$

(continuity equation).

From the matrix $M$ and its transpose

$$
M^{\prime}=\left(\begin{array}{cc}
\frac{\partial v_{x}}{\partial x} & \frac{\partial v_{y}}{\partial x} \\
\frac{\partial v_{x}}{\partial y} & \frac{\partial v_{y}}{\partial y}
\end{array}\right)
$$

we can obtain the antisymmetric matrix

$$
\begin{aligned}
M-M^{\prime} & =\left(\begin{array}{cc}
0 & \frac{\partial v_{x}}{\partial y}-\frac{\partial v_{y}}{\partial x} \\
\frac{\partial v_{y}}{\partial x}-\frac{\partial v_{x}}{\partial y} & 0
\end{array}\right) \\
& =\left(\begin{array}{rr}
0 & -\zeta \\
+\zeta & 0
\end{array}\right)
\end{aligned}
$$

where

$$
\zeta=\frac{\partial v_{y}}{\partial x}-\frac{\partial v_{x}}{\partial y}
$$

is the vorticity. We now utilize a common transformation of the hydrodynamical equations through the substitution

$$
\begin{aligned}
& M_{x y}=\frac{\partial v_{x}}{\partial y}=\frac{\partial v_{y}}{\partial x}-\zeta, \\
& M_{y x}=\frac{\partial v_{y}}{\partial x}=\frac{\partial v_{x}}{\partial y}+\zeta
\end{aligned}
$$

for the off-diagonal elements of the matrix of velocity derivatives (8.2); this substitution with $v^{2}=v_{x}^{2}+v_{y}^{2}$ yields

$$
\left\{\begin{array}{l}
\frac{\partial}{\partial x}\left(\frac{v^{2}}{2}\right)-v_{y} \zeta=-\frac{1}{\rho} \frac{\partial p}{\partial x} \\
\frac{\partial}{\partial y}\left(\frac{v^{2}}{2}\right)+v_{x} \zeta=-\frac{1}{\rho} \frac{\partial p}{\partial y}
\end{array}\right.
$$

for the hydrodynamical equations.

In the following discussion we make use of another transformation and note that the matrix elements on the main diagonal in (8.2), i.e., the elements

$$
M_{x x}=\frac{\partial v_{x}}{\partial x}, \quad M_{y y}=\frac{\partial v_{y}}{\partial y},
$$

can be written, with the aid of the continuity equation (8.3), as

$$
M_{x x}=-\frac{\partial v_{y}}{\partial y}, \quad M_{y y}=-\frac{\partial v_{x}}{\partial x}
$$

so that the matrix (8.2) becomes

$$
M=\left(\begin{array}{rr}
-\frac{\partial v_{y}}{\partial y} & \frac{\partial v_{x}}{\partial y} \\
\frac{\partial v_{y}}{\partial x} & -\frac{\partial v_{x}}{\partial x}
\end{array}\right)
$$

II. Orthogonality of isogons and isobars for nonunidirectional, stationary, planar flow of an ideal, incompressible fluid

For inhomogeneous fbw fields the matrices (8.2) and (8.10) are not null-matrices, and with the matrix (8.10) the hydrodynamical momentum equations are

$$
\left\{\begin{array}{l}
-v_{x} \frac{\partial v_{y}}{\partial y}+v_{y} \frac{\partial v_{x}}{\partial y}=-\frac{1}{\rho} \frac{\partial p}{\partial x} \\
+v_{x} \frac{\partial v_{y}}{\partial x}-v_{y} \frac{\partial v_{x}}{\partial x}=-\frac{1}{\rho} \frac{\partial p}{\partial y}
\end{array}\right.
$$


We define the direction of the wind vector $\mathbf{v}=$ $\left(v_{x}, v_{y}\right)$ as the angle with respect to the positive $x$-axis, so that

$$
v_{x}=v \cos \theta, \quad v_{y}=v \sin \theta, \quad \frac{v_{y}}{v_{x}}=\tan \theta .
$$

The wind direction field $\theta(x, y)$ can be illustrated through the isolines $\theta(x, y)=$ const $\left(=c_{1}, c_{2}, \cdots\right)$, which are points with equal values of wind direction; these isolines can be constructed graphically from the streamline field [see, for example, J. W. SANDSTRÖM, W. HorT, F. E. RELTON, Ref. (3-5)]. In dynamic meteorology the lines $\theta(x, y)=$ const are referred to as "isogons." We exclude the special case in which the function $\theta(x, y)$ has the same value at all field points, and hence only treat the case of non-unidirectional fbw fields.

We now have

$$
\left\{\begin{array}{l}
-v_{x} \frac{\partial v_{y}}{\partial y}+v_{y} \frac{\partial v_{x}}{\partial y}=-v_{x}^{2} \frac{\partial}{\partial y}\left(\frac{v_{y}}{v_{x}}\right)=-v^{2} \frac{\partial \theta}{\partial y} \\
+v_{x} \frac{\partial v_{y}}{\partial x}-v_{y} \frac{\partial v_{x}}{\partial x}=+v_{x}^{2} \frac{\partial}{\partial x}\left(\frac{v_{y}}{v_{x}}\right)=+v^{2} \frac{\partial \theta}{\partial x}
\end{array}\right.
$$

and the hydrodynamical momentum equations (8.11) take the form:

$$
\left\{\begin{array}{l}
+\rho v^{2} \frac{\partial \theta}{\partial y}=+\frac{\partial p}{\partial x} \\
-\rho v^{2} \frac{\partial \theta}{\partial x}=+\frac{\partial p}{\partial y}
\end{array}\right.
$$

so that

$$
\frac{\partial \theta}{\partial x} \frac{\partial p}{\partial x}+\frac{\partial \theta}{\partial y} \frac{\partial p}{\partial y}=0
$$

and we have the following result:

In the planar flow of an ideal incompressible fluid the isogons and isobars form an orthogonal trajectory system in the stationary case.

In accordance with its derivation, this statement applies to both planar rotational fbws as well as planar potential fbws, for the acceptance of the existence of a velocity potential was not required; exempted from the statement are the the singular points of the function $\theta(x, y)$ [R. ZURMÜHL, Ref. (6)].

III. Orthogonality of isogons and isolines of potential energy when there exists a potential for external forces

If $X, Y$ denote the components of the external force

$$
(X, Y)=-\left(\frac{\partial}{\partial x}, \frac{\partial}{\partial y}\right) \Phi
$$

expressed in terms of the potential $\Phi,(8.15)$ changes to a form in which the pressure $p$ is replaced by the potential energy $E_{\text {pot }}=\rho \Phi+p$ :

$$
\frac{\partial \theta}{\partial x} \frac{\partial E_{\mathrm{pot}}}{\partial x}+\frac{\partial \theta}{\partial y} \frac{\partial E_{\mathrm{pot}}}{\partial y}=0
$$

and the hydrodynamical momentum equations (8.14) can be written as

$$
\left\{\begin{aligned}
\rho v^{2} \frac{\partial \theta}{\partial y} & =\frac{\partial}{\partial x}(\rho \Phi+p) \\
-\rho v^{2} \frac{\partial \theta}{\partial x} & =\frac{\partial}{\partial y}(\rho \Phi+p)
\end{aligned}\right.
$$

\section{Orthogonality of isogons and isotachs in station- ary, two-dimensional, potential flow}

Suppose there exists a potential fbw:

$$
\left(v_{x}, v_{y}\right)=\left(\frac{\partial}{\partial x}, \frac{\partial}{\partial y}\right) \varphi, \quad \zeta \equiv 0,
$$

so that in the stationary case the Bernoulli equation is

$$
\rho \frac{v^{2}}{2}+E_{\text {pot }}=\rho \frac{v^{2}}{2}+\rho \Phi+p=\text { const. }
$$

Eliminating $E_{\text {pot }}$ from (8.16) with the aid of the Bernoulli equation, we obtain

$$
\frac{\partial \theta}{\partial x} \frac{\partial v}{\partial x}+\frac{\partial \theta}{\partial y} \frac{\partial v}{\partial y}=0
$$

The curves $v(x, y)=$ const $\left(=v_{1}, v_{2}, \cdots\right)$ are the isotachs. In non-unidirectional, stationary, planar, potential flow of an ideal incompressible fluid the isogons and isotachs must form an orthogonal trajectory system.

\section{Unidirectional, planar, stationary potential flow}

In the case that $\theta(x, y)=$ const in the entire field, then $\partial \theta / \partial x=\partial \theta / \partial y=0$, so that the hydrodynamical equations (8.17) yield: $\rho \Phi+p=$ const; with the aid of the Bernoulli equation (8.19), it follows that: $v=$ const, and the potential fbw is homogeneous.

\section{Conclusions}

The construction of isogons for non-unidirectional, stationary, planar fbw of an ideal incompressible fluid is made possible when only the streamlines (and not necessarily the streamfunction) are given, so that the isogons are oriented perpendicular to

a) the pressure field, if no other force field exists, 
b) the potential energy field, if another force field exists,

c) the isotach field, if a potential fbw exists, so that the isolines in each case form an orthogonal trajectory system with the isogons.

For a given streamfunction, the streamlines are determined; then the isolines in each case (i.e., pressure, potential energy, or isotachs) can also be determined because in each case their gradient is completely determined.

\section{References}

1. M. LagALly, Ideale Flüssigkeiten. Handbuch der Physik (Geiger-Scheel), Bd. VII, Berlin 1927, S. 3.
2. L. M. MiLne-Thomson, Theoretical Hydrodynamics. Third Edition, London 1955, pag. 100.

3. J. W. SANDSTRÖM, Über die Bewegung der Flüssigkeiten. Annalen der Hydrographie und Maritimen Meteorologie, Berlin 1909, S. 242.

4. W. HORT, Die Differentialgleichungen des Ingenieurs. 2. Aufl. Berlin 1925, S. 222.

5. F. E. Relton, Applied Differential Equations. London and Glasgow 1948, pag. 100.

6. R. ZuRMÜHL, Praktische Mathematik für Ingenieure und Physiker. Berlin-Göttingen-Heidelberg 1953, S. 324.

\section{ERTEL (1957): A general relationship between wind velocity and the hydrody- namical field intensity in the atmosphere}

\section{Summary}

A general relationship between the atmospheric wind velocity and the hydrodynamical fi eld intensity can be obtained if we take into account that the acceleration vector is always located in the osculator plane of the trajectory. The relationship is easily obtained from the dynamical condition in the direction of the binormal unit vector of the moving trihedron. The same equation is obtained for the geostrophic wind, the only difference being the meaning of the two unit perpendicular vectors. This equation is valid for all frictionless atmospheric flows.

\section{Introduction}

In atmospheric mechanics, or dynamical meteorology, which studies the movements of the atmosphere's air masses, we deal fundamentally with the velocity field (v). If the air moves without friction, this field is related to the vectorial field $\mathbf{F}$ of the forces of gravity $(-\nabla \Phi)$ and pressure gradient $\left(-\frac{1}{\rho} \nabla p\right)$ per unit mass, that is,

$$
\mathbf{F}=-\nabla \Phi-\frac{1}{\rho} \nabla p
$$

through the Coriolis force

$$
\mathbf{C}=2 \mathbf{v} \times \boldsymbol{\omega}
$$

and the acceleration

$$
\frac{d \mathbf{v}}{d t}=\left(\frac{\partial}{\partial t}+\mathbf{v} \cdot \nabla\right) \mathbf{v}
$$

of the particles.
Euler's equations of motion, which can be summarized in a single vectorial equation

$$
\left(\frac{\partial}{\partial t}+\mathbf{v} \cdot \nabla\right) \mathbf{v}-2 \mathbf{v} \times \boldsymbol{\omega}=-\nabla \Phi-\frac{1}{\rho} \nabla p,
$$

yield the general relationship between the three vectors, $d \mathbf{v} / d t, \mathbf{C}$, and $\mathbf{F}$. Since $\mathbf{F}=\mathbf{0}$ (null vector) should be the equilibrium condition, that is, the case of a hydrostatic field, the force $\mathbf{F} \neq 0$ is referred to as the intensity of the hydrodynamical field (in German: hydrodynamische Feldstärke).

In order to close the system of equations that determine the atmospheric motion, we must add the continuity equation and a thermodynamical relationship. However, we know that this system of equations is among the most complex systems in theoretical physics, because the equations are nonlinear differential equations.

Nevertheless, it so happens in dynamical meteorology that a number of air motion problems can be studied in an approximate sense, if one assumes that the acceleration is always small. An example of this is the geostrophic wind (see, for instance, P. CARRASCO, Meteorologí a, MÉxico 1945, pages 175-176; D. BRUNT, Climatologí a, Buenos Aires-MÉxico 1948, pages 194202; S. PetTerssen, Introducción a la Meteorologí a, Buenos Aires-México 1951, pages 156-161).

\section{The problem}

Let us consider a motion in the horizontal plane. We fix a rectangular coordinate system $(x, y, z)$, whose 
positive $z$-axis is vertical. Then $\mathbf{v}=\left(v_{x}, v_{y}, 0\right)$. We assume that in the fundamental hydrodynamical equations the acceleration can be neglected when compared to the Coriolis force. Now the horizontal motion can be expressed as

$$
-2 \omega_{z} v_{y}=F_{x}, \quad+2 \omega_{z} v_{x}=F_{y},
$$

if we adopt the right-hand rule as the direct or positive trihedron. Let us rotate the coordinate axes trihedron around the $+z$ axis until the $+x$ axis coincides with the wind direction, that is,

$$
\mathbf{v}=\left(v_{x}=v=|\mathbf{v}|, 0,0\right) .
$$

As a result, we have

$$
v=\frac{1}{2} \frac{F_{y}}{\omega_{z}}
$$

which is nothing but the well-known formula for the geostrophic wind velocity, where $\omega_{z}=|\boldsymbol{\omega}| \cos (\pi / 2-$ $\varphi)=\omega \sin \varphi$ is the vertical component of the Earth's angular velocity at the northern latitude $\varphi$. Using the unit vectors $\mathbf{j}, \mathbf{k}$ in the $y, z$ directions, respectively, we can write the previous equation as

$$
v=\frac{1}{2} \frac{(\mathbf{F} \cdot \mathbf{j})}{(\boldsymbol{\omega} \cdot \mathbf{k})} .
$$

Now let us consider the following problem. Given the intensity of the hydrodynamic field $\mathbf{F}$ at a certain point in the atmosphere (and we have yet to introduce in the calculation a rigorous treatment of the influence of acceleration) is it possible to determine the wind velocity $v=|\mathbf{v}|$ at that point with the help of an equation that is similar to the equation obtained for the case of the geostrophic wind?

\section{Solution}

First we need to study in more detail the acceleration in Euler's equations of motion. The $d / d t$ operator represents a derivative following the motion of the particle trajectories, and if $d s=v d t$ is an infinitesimal distance along the trajectory, then

$$
\frac{d \mathbf{v}}{d t}=v \frac{d \mathbf{v}}{d s} .
$$

Moreover, let $\mathbf{T}$ be the unit tangent in the direction of the motion of the particle, that is, $\mathbf{v}=\mathbf{T} v$. Euler's vector equation of motion is therefore

$$
\mathbf{T} \frac{d}{d s}\left(\frac{v^{2}}{2}\right)+v^{2} \frac{d \mathbf{T}}{d s}+2 v \boldsymbol{\omega} \times \mathbf{T}=\mathbf{F} .
$$

After a scalar product of this equation by the following vectorial product

$$
\mathbf{L}=\frac{\mathbf{T} \times \frac{d \mathbf{v}}{d s}}{v\left|\frac{d \mathbf{T}}{d s}\right|}=\frac{\mathbf{T} \times \frac{d \mathbf{T}}{d s}}{\left|\frac{d \mathbf{T}}{d s}\right|}
$$

we obtain

$$
2 v(\boldsymbol{\omega} \cdot \mathbf{M})=(\mathbf{F} \cdot \mathbf{L}),
$$

where $\mathbf{M}$ is the vector

$$
\mathbf{M}=\mathbf{T} \times \mathbf{L} .
$$

We then effectively have the relationship

$$
v=\frac{1}{2} \frac{(\mathbf{F} \cdot \mathbf{L})}{(\boldsymbol{\omega} \cdot \mathbf{M})}
$$

which has the form of the solution needed because, as we will show below, the right hand side of the equation obeys all of the conditions established. This is exactly the same equation as the equation for the geostrophic wind. The only difference stems from the meaning of $\mathbf{L}$ and $\mathbf{M}$.

It is immediately apparent that $\mathbf{L}$ is in the normal plane, that is, in the plane perpendicular to T. $\mathbf{M}$ is also in this plane:

$$
\mathbf{L} \cdot \mathbf{T}=0, \quad \mathbf{M} \cdot \mathbf{T}=0 .
$$

Moreover $\mathbf{L}$ is orthogonal to $\mathbf{M}=\mathbf{T} \times \mathbf{L}$ :

$$
\mathbf{L} \cdot \mathbf{M}=0,
$$

and both are unit vectors:

$$
|\mathbf{L}|=|\mathbf{M}|=1 .
$$

Finally, we are ready to find the directions of the unit vectors $\mathbf{L}$ and $\mathbf{M}$ in the normal plane with the help of a moving trihedron of three perpendicular unit vectors, namely: the unit tangent $\mathbf{T}$, the binormal unit $\mathbf{B}$ and the principal normal unit $\mathbf{N}$. Defining $\mathbf{T}, \mathbf{B}, \mathbf{N}$ as the right-handed trihedron, we can write

$$
\mathbf{T}=\mathbf{B} \times \mathbf{N}, \quad \mathbf{B}=\mathbf{N} \times \mathbf{T}, \quad \mathbf{N}=\mathbf{T} \times \mathbf{B},
$$

and introducing a well-known equation from the differential geometry

$$
\frac{d \mathbf{T}}{d s}=-\kappa \mathbf{N}
$$


where $\kappa$ is the trajectory curvature, we have

$$
\left|\frac{d \mathbf{T}}{d s}\right|=\kappa, \quad \mathbf{T} \times \frac{d \mathbf{T}}{d s}=\kappa \mathbf{B},
$$

that is

$$
\mathbf{L}=\mathbf{B} \quad \text { and } \quad \mathbf{M}=\mathbf{N}
$$

(see, for instance, D. J. STRUiK, Geometrí a Diferencial Clássica, Madrid 1955, pages 17-23; F. J. GuILlÉN, Cálculo Tensorial, México 1957, pages 34-38).

The general relationship between the wind velocity and the intensity of the hydrodynamical field in the atmosphere becomes:

$$
v=\frac{1}{2} \frac{(\mathbf{F} \cdot \mathbf{B})}{(\boldsymbol{\omega} \cdot \mathbf{N})}
$$

or, if we introduce cartesian coordinates,

$$
v=\frac{1}{2} \frac{\left(F_{x} B_{x}+F_{y} B_{y}+F_{z} B_{z}\right)}{\left(\omega_{x} N_{x}+\omega_{y} N_{y}+\omega_{z} N_{z}\right)}
$$

from which we see that the geostrophic wind is a special case, for instance,

$$
\mathbf{B}=(0,1,0), \quad \mathbf{N}=(0,0,1) .
$$

\section{Additional considerations}

It therefore seems that the real wind velocity is determined by six parameters, the components of the vectors $\mathbf{B}=\left(B_{x}, B_{y}, B_{z}\right)$ and $\mathbf{N}=\left(N_{x}, N_{y}, N_{z}\right)$. It is important to point out that there are three relationships among them:

$$
\begin{gathered}
B_{x}^{2}+B_{y}^{2}+B_{z}^{2}=1, \\
N_{x}^{2}+N_{y}^{2}+N_{z}^{2}=1, \\
B_{x} N_{x}+B_{y} N_{y}+B_{z} N_{z}=0,
\end{gathered}
$$

and therefore only three of those parameters are completely independent, a number which is equal to the number of components of the acceleration $d \mathbf{v} / d t=$ $\left(d v_{x} / d t, d v_{y} / d t, d v_{z} / d t\right)$. It seems at first sight that the set of three independent parameters can be considered directly equivalent to the acceleration. However, a more careful review will show that this is not the case. We will write the general relationship as

$$
v=\frac{1}{2} \frac{(\mathbf{F} \cdot \mathbf{B})}{(\boldsymbol{\omega} \cdot \mathbf{N})}=\frac{1}{2} \frac{\mathbf{F} \cdot\left(\mathbf{T} \times \frac{d \mathbf{v}}{d t}\right)}{\boldsymbol{\omega} \cdot\left(\mathbf{T} \times\left(\mathbf{T} \times \frac{d \mathbf{v}}{d t}\right)\right)} .
$$

This equation yields a relationship between those magnitudes; however, according to a kinematics theorem, the three vectors $d \mathbf{v} / d t, \mathbf{T}$ and $\mathbf{N}$ are linearly dependent:

$$
\frac{d \mathbf{v}}{d t}=\mathbf{T}\left(\frac{d \mathbf{v}}{d t}\right)_{T}+\mathbf{N}\left(\frac{d \mathbf{v}}{d t}\right)_{N},
$$

that is, the acceleration vector is always located in the osculating plane of the trajectory (see, for instance, C. Mataix Aracil, Cálculo Vectorial Intrí nseco, Third Edition, Madrid 1951, page 49). Combined with the equation for $v$, this last equation produces

$$
v=\frac{1}{2} \frac{\mathbf{F} \cdot(\mathbf{T} \times \mathbf{N})}{\boldsymbol{\omega} \cdot(\mathbf{T} \times(\mathbf{T} \times \mathbf{N}))}=\frac{1}{2} \frac{(\mathbf{F} \cdot \mathbf{B})}{(\boldsymbol{\omega} \cdot \mathbf{N})}=\frac{1}{2} \frac{F_{B}}{\omega_{N}} .
$$

The scalar factor $(d \mathbf{v} / d t)_{N}$ can be omitted. We therefore obtain an equation that does not explicitly contain the acceleration, but that besides $\mathbf{F}$ and $\boldsymbol{\omega}$, only contains purely geometric quantities.

Taking into consideration the relationships between the unit vectors $\mathbf{T}, \mathbf{B}$, and $\mathbf{N}$, we easily recognize that the following equations are also valid:

$$
\begin{aligned}
& v=\frac{1}{2} \frac{(\mathbf{F} \cdot(\mathbf{N} \times \mathbf{T}))}{(\boldsymbol{\omega} \cdot \mathbf{N})}, \\
& v=\frac{1}{2} \frac{(\mathbf{F} \cdot \mathbf{B})}{(\boldsymbol{\omega} \cdot(\mathbf{T} \times \mathbf{B}))} .
\end{aligned}
$$

Each equation in these two cases contains two of the three independent parameters; $\mathbf{T}$ determines the normal plane. For instance, if we fix the normal plane, that is, the vector $\mathbf{T}$ through its two independent components, we will still need a direction in this normal plane to determine $\mathbf{B}$ or $\mathbf{N}$.

\section{Simpler solution}

We'll finish with a second demonstration:

If we now write Euler's equation of motion as follows:

$$
\mathbf{T}\left(\frac{d \mathbf{v}}{d t}\right)_{T}+\mathbf{N}\left(\frac{d \mathbf{v}}{d t}\right)_{N}+2 v(\boldsymbol{\omega} \times \mathbf{T})=\mathbf{F}
$$

and take the scalar product with the binormal unit vector B, we find

$2 v(\boldsymbol{\omega} \times \mathbf{T}) \cdot \mathbf{B}=2 v \boldsymbol{\omega} \cdot(\mathbf{T} \times \mathbf{B})=2 v(\boldsymbol{\omega} \cdot \mathbf{N})=\mathbf{F} \cdot \mathbf{B}$.

As seen above, this equation of motion in the binormal unit direction of the moving trihedron yields also the relationship between the wind velocity and the intensity of the atmospheric hydrodynamic field. 


\section{ERTEL (1960a): Theorem on hydrodynamical material invariants}

\section{Summary}

The following theorem is easily demonstrated: The product of the specific volume by the functional determinant of three material invariants with respect to orthogonal cartesian spatial coordinates is also a material invariant.

\section{Symbols}

We use tensor notation and omit the summation symbol for indices that appear twice. We define the following symbols: $x_{i}(i=1,2,3)=$ rectangular cartesian coordinates, $v_{i}(i=1,2,3)=$ components of the fuid velocity, $\delta_{j k}(j, k=1,2,3)=$ substitution tensor, $(\cdots)_{, j}=$ partial derivative of $(\cdots)$ with respect to $x_{j}$, $d(\cdots) / d t=\partial(\cdots) / \partial t+v_{j}(\cdots)_{, j}=$ material derivative of $(\cdots), \sigma=1 / \rho=$ specific volume ( $\rho=$ density) of the fluid, and $t=$ time.

\section{Concept of a material invariant}

Let $f\left(x_{1}, x_{2}, x_{3}, t\right)$ be a property of a fluid particle; then

$$
\frac{d f}{d t}=\left(v_{1} \frac{\partial}{\partial x_{1}}+v_{2} \frac{\partial}{\partial x_{2}}+v_{3} \frac{\partial}{\partial x_{3}}\right) f+\frac{\partial f}{\partial t}
$$

is called a material derivative because $d f / d t$ measures the variation of $f$ as a function of time following the fluid particle. If $F\left(x_{1}, x_{2}, x_{3}, t\right)$ is conserved following the parcel, then $d F / d t=0$ and therefore we can write

$$
\left(v_{1} \frac{\partial}{\partial x_{1}}+v_{2} \frac{\partial}{\partial x_{2}}+v_{3} \frac{\partial}{\partial x_{3}}\right) F=-\frac{\partial F}{\partial t},
$$

or simply

$$
v_{j} F_{, j}=-\frac{\partial F}{\partial t}
$$

using tensor notation.

In this case, $F$ is conserved along the trajectory of a moving particle. For this reason $F$ is also called a material constant. There are many applications for a material invariant in meteorology and oceanography.

\section{A theorem on material invariants}

Let $J_{k}(k=1,2,3)$ be three material invariants. The Jacobian ( $k=$ subindex of rows, $j=$ subindex of columns)

$$
J=\left|J_{k, j}\right|=\frac{\partial\left(J_{1}, J_{2}, J_{3}\right)}{\partial\left(x_{1}, x_{2}, x_{3}\right)}
$$

is not equal to zero, that is, $J_{1}, J_{2}$, and $J_{3}$ are independent.
Moreover, it is necessary that the following three conditions are met:

$$
v_{j} J_{k, j}=-\frac{\partial J_{k}}{\partial t} .
$$

Denote the cofactor of $J_{k, i}$ in $J$ as $\stackrel{*}{J_{k i}}$. Using this notation we have

$$
\stackrel{*}{J}_{k i} J_{k, j}=\delta_{i j} J= \begin{cases}J, & \text { if } i=j, \\ 0, & \text { if } i \neq j,\end{cases}
$$

and as solutions of the system of equations in (10.4), with $v_{i}(i=1,2,3)$ as unknowns and using (10.5), we obtain:

$$
J v_{i}=-\frac{\partial J_{k}}{\partial t} J_{k i}
$$

The divergence of the vector $J v_{i}$ is then given by

$$
\left(J v_{i}\right)_{, i}=-\frac{\partial J}{\partial t},
$$

because

$$
\left(\stackrel{*}{J}_{k i}\right)_{, i}=\stackrel{*}{J}_{k i, i} \equiv 0
$$

for all $k$; moreover

$$
\frac{\partial J_{k, i}}{\partial t} \stackrel{*}{J}_{k i}=\frac{\partial J}{\partial t}
$$

is the derivative of the determinant $J$ with respect to $t$.

From the continuity equation

$$
\frac{d \sigma}{d t}=\sigma v_{i, i}
$$

equation (10.7) can be written as

$$
\sigma\left(\frac{\partial J}{\partial t}+v_{i} J_{, i}\right)+J \frac{d \sigma}{d t}=0,
$$

which can be rewritten as:

$$
\frac{d}{d t}(\sigma J)=\frac{d}{d t}\left\{\sigma \frac{\partial\left(J_{1}, J_{2}, J_{3}\right)}{\partial\left(x_{1}, x_{2}, x_{3}\right)}\right\}=0 .
$$

Therefore, the following theorem is verified:

The product of the specific volume by the functional determinant of three material invariants with respect to orthogonal cartesian spatial coordinates is also a material invariant.

Analogous results are obtained in the general case of $n$ material derivatives $J_{k}\left(x_{1}, x_{2}, x_{3}, \ldots, x_{n}, t\right)$ $(k=1,2,3, \ldots, n)$ with $n$ orthogonal cartesian coordinates of an $n$-dimensional space, assuming that $J_{1}, J_{2}, J_{3}, \ldots, J_{n}$ are independent. 


\section{Meteorological examples}

Isentropic motion in vertical columns of the atmosphere is an example of an application of equation (10.7). Assume an isentropic air motion in the vertical direction, $x_{3}=z$. All particles move parallel to the vertical axis without lateral expansion or contraction and without change of specific entropy, $S$, so that there are three material invariants

$$
\left.\begin{array}{l}
J_{1}=x_{1}=x, \\
J_{2}=x_{2}=y, \\
J_{3}=S(x, y, z, t) .
\end{array}\right\}
$$

These functions are independent in the region of study. The functional determinant (10.3) can be written as

$$
J=\frac{\partial(x, y, S)}{\partial(x, y, z)}=\frac{\partial S}{\partial z},
$$

and equation (10.12) gives the well-known result

$$
\frac{d}{d t}\left(\sigma \frac{\partial S}{\partial z}\right)=0
$$

A so-called Margules material invariant, $\sigma(\partial S / \partial z)$, appears in this equation.

The following equality is sometimes convenient

$$
J=\frac{\partial\left(J_{1}, J_{2}, J_{3}\right)}{\partial\left(x_{1}, x_{2}, x_{3}\right)}=\left(\nabla J_{1} \times \nabla J_{2}\right) \cdot \nabla J_{3}
$$

(see, for example, ErTel [1], Morán SAMAniego [2]).

\section{References}

[1] H. ERTEL, H., Winddivergenz auf Isobarenflächen und Luftdruckänderung. Met. Z. 60 (1943), 188-191.

[2] Morán Samaniego, F., Apuntes de Termodinámica de la Atmósfera, Madrid 1944, $70-81$.

\section{Ertel (1960b): Relation between a material derivative and a given spatial divergence in hydrodynamics}

\section{Summary}

We derive a transformation that shows how the material derivative of any function can be expressed as a spatial divergence multiplied by the fluid's specifi c volume. This relationship shows, for instance, the different forms of the continuity equation (Euler, Lagrange) as special cases.

\section{Symbols, notation, and fundamental concepts}

We define the following symbols: $x_{i}(i=1,2,3)=$ rectangular cartesian coordinates, $v_{i}(i=1,2,3)=$ components of the fluid velocity, $t=$ time, $\sigma=$ specific volume, $\rho=$ density $(=1 / \sigma), F_{i}(i=1,2,3)=$ components of the external force, $p_{i j}(i, j=1,2,3)=$ pressure tensor, and $\delta_{i j}(i, j=1,2,3)=$ substitution tensor.

In order to simplify the notation we use the summation rule of tensorial calculus in which the use of index repetition indicates summation. Indices that are not repeated will take on values of $1,2,3$ successively so that there is a total of three different equations.

It is convenient to write $\psi_{, j}$ instead of $\partial \psi / \partial x_{j}$. With this notation $\Theta_{k, j}=\partial \Theta_{k} / \partial x_{j}, p_{i j, j}=\partial p_{i j} / \partial x_{j}$, etc. (see, for instance, VoGEL [4]).

Let $\psi=\psi\left(x_{1}, x_{2}, x_{3}, t\right)$ be a differentiable function whose physical meaning is the component of a tensor of any order. The operator

$$
\frac{\partial \psi}{\partial t}+v_{j} \psi_{, j}=\frac{d \psi}{d t}
$$

is the so-called material derivative of $\psi$ with respect to $t$. We know that (11.1) represents a derivative following the motion of a particle which at time $t$ is located at $x_{j}$ and has velocity $d x_{j} / d t=v_{j}(j=1,2,3)$.

Introducing the continuity equation

$$
\frac{d \sigma}{d t}=\sigma v_{j, j}
$$

we can write the material derivative in (11.1) as

$$
\frac{d \psi}{d t}=\sigma\left\{\left(\rho v_{j} \psi\right)_{, j}+\frac{\partial(\rho \psi)}{\partial t}\right\}
$$

We see that the material derivative is here expressed as a four-dimensional divergence (a spatio-temporal divergence) multiplied by the fluid's specific volume. Equation (11.3) is often found in conservation principles of theoretical physics (see, for instance, SCHRÖDINGER [3]). 
Euler's fundamental hydrodynamical equations contain both material derivatives and spatial divergences:

$$
\frac{d v_{i}}{d t}+\sigma p_{i j, j}=F_{i}
$$

The continuity equation (11.2) also has this property. It is therefore convenient to express all material derivatives as spatial divergences. In the next section, we will show that this transformation is possible with the help of functional determinants (Jacobian and Hessian).

\section{Transformation of the material derivative to a spa- tial divergence}

After the appropriate choice of three functions

$$
\Theta_{k}=\Theta_{k}\left(x_{1}, x_{2}, x_{3}, t\right) \quad(k=1,2,3)
$$

it is possible to express a function $\psi=\psi\left(x_{1}, x_{2}, x_{3}, t\right)$ as the Jacobian $J=J\left(\Theta_{1}, \Theta_{2}, \Theta_{3}\right)$ multiplied by the specific volume, as follows:

$$
\psi=\sigma \frac{\partial\left(\Theta_{1}, \Theta_{2}, \Theta_{3}\right)}{\partial\left(x_{1}, x_{2}, x_{3}\right)}=\sigma\left|\Theta_{k, j}\right|=\sigma J
$$

( $k=$ subindex of rows, $j=$ subindex of columns). This kind of representation is always possible when $\Theta_{1}, \Theta_{2}, \Theta_{3}$ are independent, that is:

$$
J=\left|\Theta_{k, j}\right| \neq 0 .
$$

If we designate as $\stackrel{*}{\Theta}_{k j}$ the adjoint determinant of $\Theta_{k, j}$ in $J=\left|\Theta_{k, j}\right|$, a well-known property of the determinants tells us that

$$
\stackrel{*}{\Theta}_{k i} \Theta_{k, j}=\delta_{i j} J
$$

Consider now the identities

$$
\frac{\partial \Theta_{k}}{\partial t}+v_{j} \Theta_{k, j}=\frac{d \Theta_{k}}{d t} .
$$

If we multiply these equations by $\stackrel{*}{\Theta}_{k i}$, and sum over all $k$, use of (11.7) gives

$$
\frac{\partial \Theta_{k}}{\partial t} \stackrel{*}{\Theta}_{k i}+v_{i} J=\stackrel{*}{\Theta}_{k i} \frac{d \Theta_{k}}{d t} .
$$

From (11.9) we'd like to obtain the spatial divergence; we then have

$$
\frac{\partial J}{\partial t}+\left(v_{i} J\right)_{, i}=\left(\stackrel{*}{\Theta}_{k i} \frac{d \Theta_{k}}{d t}\right)_{, i}
$$

because for every $k$

$$
\left(\stackrel{*}{\Theta}_{k i}\right)_{, i}=\stackrel{*}{\Theta}_{k i, i}=0,
$$

while

$$
\frac{\partial \Theta_{k, i}}{\partial t} \stackrel{*}{\Theta}_{k i}=\frac{\partial J}{\partial t}
$$

is nothing but the partial derivative of the functional determinant $J$ with respect to $t$.

Considering the continuity equation (11.2) we then have, according to (11.10):

$$
\sigma\left(\frac{\partial J}{\partial t}+v_{i} J_{, i}\right)+J \frac{d \sigma}{d t}=\sigma\left(\Theta_{k j} \frac{d \Theta_{k}}{d t}\right)_{, j}
$$

and taking equation (11.1) into account, we obtain the needed transformation:

$$
\frac{d}{d t}\left\{\sigma \frac{\partial\left(\Theta_{1}, \Theta_{2}, \Theta_{3}\right)}{\partial\left(x_{1}, x_{2}, x_{3}\right)}\right\}=\sigma\left(\stackrel{*}{\Theta}_{k j} \frac{d \Theta_{k}}{d t}\right)_{, j}
$$

or

$$
\frac{d \psi}{d t}=\sigma\left(\stackrel{*}{\Theta}_{k j} \frac{d \Theta_{k}}{d t}\right)_{, j}
$$

if we remember that $\psi$ is given by (11.5).

Through this relationship it is possible to transform the material derivative of a function $\psi=$ $\sigma J\left(\Theta_{1}, \Theta_{2}, \Theta_{3}\right)$ into a spatial divergence. This transformation is also valid for a Euclidian space of any dimension.

It is important to point out that in (11.15) the set of functions $\Theta_{k}(k=1,2,3)$ contains two arbitrary functions, providing $\infty^{2}$ ways of representation if we express $\psi / \sigma$ using the Jacobian in (11.5). If we assume that $\psi / \sigma, \Theta_{1}$ and $\Theta_{2}$ are given, then equation (11.5) determines $\Theta_{3}$ through a first order partial differential equation.

For clarity let us consider the function $\psi=\sigma$, and $\Theta_{1}=x_{1}, \Theta_{2}=x_{2}$. Then, equation (11.5) requires that $\Theta_{3}=x_{3}$. Therefore, $\stackrel{*}{\Theta}_{k j}=\delta_{j k}$, and equation (11.14) yields

$$
\frac{d \sigma}{d t}=\sigma\left(\delta_{j k} \frac{d x_{k}}{d t}\right)_{, j}=\sigma v_{j, j}
$$

that is, the transformation in (11.15) yields Euler's continuity equation.

If we assume that $d \Theta_{k} / d t$ is a function of $\Theta_{1}, \Theta_{2}$ and $\Theta_{3}$, we can establish that

$$
\left(\stackrel{*}{\Theta}_{k j} \frac{d \Theta_{k}}{d t}\right)_{, j}=J \frac{\partial}{\partial \Theta_{j}}\left(\frac{d \Theta_{j}}{d t}\right)
$$


in virtue of

$$
\begin{aligned}
\stackrel{*}{\Theta}_{k j}\left(\frac{d \Theta_{k}}{d t}\right)_{, j} & =\stackrel{*}{\Theta}_{k j} \Theta_{i, j} \frac{\partial}{\partial \Theta_{i}}\left(\frac{d \Theta_{k}}{d t}\right) \\
= & \delta_{i k} J \frac{\partial}{\partial \Theta_{i}}\left(\frac{d \Theta_{k}}{d t}\right)=J \frac{\partial}{\partial \Theta_{j}}\left(\frac{d \Theta_{j}}{d t}\right),
\end{aligned}
$$

so that equation (11.14) becomes the equivalent form:

$$
\frac{d}{d t} \ln \left\{\sigma \frac{\partial\left(\Theta_{1}, \Theta_{2}, \Theta_{3}\right)}{\partial\left(x_{1}, x_{2}, x_{3}\right)}\right\}=\frac{\partial}{\partial \Theta_{j}}\left(\frac{d \Theta_{j}}{d t}\right),
$$

which is advantageous when two functions $\left(\Theta_{1}\right.$ and $\Theta_{2}$, for instance) from the set $\Theta_{j}(j=1,2,3)$ are canonically conjugate variables. (For further information on canonically conjugate variables in hydrodynamics see LAMB [2]). If, moreover, $\Theta_{3}$ is a material invariant, that is $d \Theta_{3} / d t=0$, we have

$$
\frac{\partial}{\partial \Theta_{j}}\left(\frac{d \Theta_{j}}{d t}\right)=0,
$$

and therefore, from (11.14a):

$$
\frac{d}{d t}\left\{\sigma \frac{\partial\left(\Theta_{1}, \Theta_{2}, \Theta_{3}\right)}{\partial\left(x_{1}, x_{2}, x_{3}\right)}\right\}=0
$$

that is

$$
\sigma \frac{\partial\left(\Theta_{1}, \Theta_{2}, \Theta_{3}\right)}{\partial\left(x_{1}, x_{2}, x_{3}\right)}=\text { material invariant. }
$$

Our transformation also includes the following theorem: If the three independent functions $\Theta_{1}, \Theta_{2}$, and $\Theta_{3}$ are individual invariants:

$$
\frac{d \Theta_{1}}{d t}=\frac{d \Theta_{2}}{d t}=\frac{d \Theta_{3}}{d t}=0
$$

the product of the specific volume by the functional determinant of $\Theta_{1}, \Theta_{2}$ and $\Theta_{3}$ with respect to the rectangular cartesian coordinates is also an individual invariant (see ERTEL [1]).

The initial position coordinates $\stackrel{\circ}{x}_{1}=a_{1}=\Theta_{1}$, $\stackrel{\circ}{x}_{2}=a_{2}=\Theta_{2}, \stackrel{\circ}{x}{ }_{3}=a_{3}=\Theta_{3}$ at a reference time $t=0$ are material invariants:

$$
\left.\begin{array}{rl}
\frac{d \Theta_{1}}{d t} & =\frac{d a_{1}}{d t}=0, \\
\frac{d \Theta_{2}}{d t} & =\frac{d a_{2}}{d t}=0, \\
\frac{d \Theta_{3}}{d t} & =\frac{d a_{3}}{d t}=0 .
\end{array}\right\}
$$

Using (11.21), equation (11.14) becomes

$$
\frac{d}{d t}\left\{\sigma \frac{\partial\left(a_{1}, a_{2}, a_{3}\right)}{\partial\left(x_{1}, x_{2}, x_{3}\right)}\right\}=0,
$$

which is a well-known equality known as Lagrange's continuity equation.

Finally, replacing the three functions $\Theta_{k}(k=$ $1,2,3)$ with a function $\Phi$, which has the property that $\Phi_{k}=\Phi_{, k}(k=1,2,3)$, turns the Jacobian (11.6) into the Hessian

$$
H(\Phi)=\left|\Phi_{, k j}\right|=\left|\Phi_{, j k}\right| .
$$

If we designate $\stackrel{*}{\Phi}_{k j}$ as the cofactor (minor with sign) of $\Phi_{, k j}$ in $H(\Phi)$, we have

$$
\Phi_{, k i} \stackrel{*}{\Phi}_{k j}=\delta_{i j} H(\Phi)
$$

instead of (11.7). Therefore, written as

$$
\frac{d}{d t}\{\sigma H(\Phi)\}=\sigma\left(\stackrel{*}{\Phi}_{k j} \frac{d \Phi_{, k}}{d t}\right)_{, j}
$$

we have a special case of equation (11.14). Using (11.25) we can transform the material derivative of a function $\psi$ into a spatial divergence, when $\psi$ is determined by a function $\Phi$ in the Hessian $H(\Phi)=\psi / \sigma$; however, in order to determine $\Phi$ we now have a nonlinear partial differential equation. It is immediately seen that (11.14), (11.14a) and (11.25) are of the form of the continuity equation.

\section{References}

[1] ERTEL, H., Teorema sobre invariantes sustanciales de la Hidrodinámica. Gerl. Beitr. Geophys. 69 (1960), 290-293.

[2] LAmB, H., Hydrodynamics. First American edition, New York 1945, 248-249.

[3] SCHRÖDInger, E., Space-Time Structure, Cambridge 1950, 87-90.

[4] Vogel, Th., Physique mathématique classique, Paris 1956, 40-43. 


\section{ERTEL (1961): Isogons and isotachs in two-dimensional potential fbw of an incompressible fluid}

\section{Summary}

Let $\theta=$ const and $v=$ const be the isogons and isotachs that correspond to two-dimensional, irrotational, and incompressible flow. Without making use of functional theoretical methods, the following theorem can be demonstrated: a rotation from $\nabla \theta$ to $\nabla v$ along the shortest path must always be a counterclockwise rotation of magnitude $\pi / 2$, if $\theta$ is measured positive in the same direction.

\section{Introduction}

Many geophysical problems can be studied within the framework of two-dimensional, irrotational, and incompressible fluid motion. This is the kind of problem addressed here. Therefore, the description of a twodimensional coordinate system suffices.

The hydrodynamical equations are reduced to:

$$
\begin{aligned}
\frac{\partial v_{x}}{\partial t}+\left(v_{x} \frac{\partial}{\partial x}+v_{y} \frac{\partial}{\partial y}\right) v_{x} & =-\frac{\partial \Phi}{\partial x}-\frac{1}{\rho} \frac{\partial p}{\partial x} \\
\frac{\partial v_{y}}{\partial t}+\left(v_{x} \frac{\partial}{\partial x}+v_{y} \frac{\partial}{\partial y}\right) v_{y} & =-\frac{\partial \Phi}{\partial y}-\frac{1}{\rho} \frac{\partial p}{\partial y} \\
\frac{\partial v_{x}}{\partial x}+\frac{\partial v_{y}}{\partial y} & =0 \\
\frac{\partial v_{y}}{\partial x}-\frac{\partial v_{x}}{\partial y} & =0
\end{aligned}
$$

where $x, y$ are rectangular cartesian coordinates, $v_{x}, v_{y}$ the components of velocity, $\Phi$ the external force potential, $p$ the pressure, and $\rho$ the density.

In equations (12.1) and (12.2) the convective portions of the acceleration are

$$
\begin{aligned}
& B_{x}=v_{x} \frac{\partial v_{x}}{\partial x}+v_{y} \frac{\partial v_{x}}{\partial y}=\frac{\partial}{\partial x}\left(\frac{v^{2}}{2}\right), \\
& B_{y}=v_{x} \frac{\partial v_{y}}{\partial x}+v_{y} \frac{\partial v_{y}}{\partial y}=\frac{\partial}{\partial y}\left(\frac{v^{2}}{2}\right),
\end{aligned}
$$

where $v=|\mathbf{v}|$ is the absolute value of the vector $\mathbf{v}=\left(v_{x}, v_{y}\right)$.

Based on conditions (12.3) and (12.4) we will investigate the isogons and isotachs of the given hydrodynamical field.

\section{Isogons and isotachs}

Let $x, y$ be a right-handed coordinate system. Using equation (12.3) we replace $\partial v_{x} / \partial x$ with $-\partial v_{y} / \partial y$ in (12.5) and $\partial v_{y} / \partial y$ with $-\partial v_{x} / \partial x$ in (12.6) and, after some manipulation, obtain (see, for instance, [1]):

$$
\left.\begin{array}{l}
B_{x}=-v^{2} \frac{\partial \theta}{\partial y}=\frac{\partial}{\partial x}\left(\frac{v^{2}}{2}\right), \\
B_{y}=+v^{2} \frac{\partial \theta}{\partial x}=\frac{\partial}{\partial y}\left(\frac{v^{2}}{2}\right),
\end{array}\right\}
$$

where

$$
v_{x}=v \cos \theta, \quad v_{y}=v \sin \theta,
$$

implies that the angle

$$
\theta=\tan ^{-1}\left(\frac{v_{y}}{v_{x}}\right)
$$

is measured in a counterclockwise manner. The equation $\theta(x, y, t)=$ const represents an isogon and the equation $v(x, y, t)=$ const represents an isotach.

We deduce from (12.7) that

$$
\frac{\partial \theta}{\partial x} \frac{\partial v}{\partial x}+\frac{\partial \theta}{\partial y} \frac{\partial v}{\partial y}=0
$$

which is an orthogonality condition that states that isogons and isotachs are perpendicular at all points where they intersect (KOZENY [2]).

From equation (12.7) we can derive the CauchyRiemann conditions:

$$
\frac{\partial \ln v}{\partial x}=-\frac{\partial \theta}{\partial y}, \quad \frac{\partial \ln v}{\partial y}=\frac{\partial \theta}{\partial x}
$$

on the other hand

$$
\begin{aligned}
\frac{\partial B_{x}}{\partial x}+\frac{\partial B_{y}}{\partial y} & =2 v\left(\frac{\partial \theta}{\partial x} \frac{\partial v}{\partial y}-\frac{\partial \theta}{\partial y} \frac{\partial v}{\partial x}\right) \\
& =2 v|\nabla \theta||\nabla v| \sin \psi,
\end{aligned}
$$

where $\psi$ is the angle formed by $\nabla \theta$ and $\nabla v$. The angle $\psi$ is also measured counterclockwise.

Consider now the expression for the divergence $\partial B_{x} / \partial x+\partial B_{y} / \partial y$ as a function of the components of the deformation tensor. Differentiating equations (12.5) and (12.6) and taking into account condition (12.3), we obtain:

$$
\frac{\partial B_{x}}{\partial x}+\frac{\partial B_{y}}{\partial y}=\left(\frac{\partial v_{x}}{\partial x}\right)^{2}+2 \frac{\partial v_{x}}{\partial y} \frac{\partial v_{y}}{\partial x}+\left(\frac{\partial v_{y}}{\partial y}\right)^{2} .
$$

Recalling that in our case the components of the deformation tensor can be reduced, using (12.4), to

$$
\begin{aligned}
D_{x x} & =\frac{\partial v_{x}}{\partial x} \\
D_{x y}=D_{y x} & =\frac{\partial v_{x}}{\partial y}=\frac{\partial v_{y}}{\partial x}, \\
D_{y y} & =\frac{\partial v_{y}}{\partial y}
\end{aligned}
$$


we obtain the formula:

$$
\frac{\partial B_{x}}{\partial x}+\frac{\partial B_{y}}{\partial y}=D_{x x}^{2}+2 D_{x y}^{2}+D_{y y}^{2} .
$$

Comparing equations (12.12) and (12.15) we obtain

$$
2 v|\nabla \theta||\nabla v| \sin \psi=D_{x x}^{2}+2 D_{x y}^{2}+D_{y y}^{2} .
$$

It is easily seen that the condition for the first member of this equation is

$$
\sin \psi>0
$$

since it is necessary to exclude the exceptional case when $D_{x x}, D_{x y}$, and $D_{y y}$ simultaneously vanish at each point of the field, which describes the movement of a rigid body without rotation.

The orthogonality condition (12.10) of the vectors $\nabla \theta$ and $\nabla v$ determines two possible values for $\psi$ :

$$
\psi= \pm \frac{\pi}{2},
$$

but the inequality in equation (12.17) can only be satisfied if

$$
0<\psi<\pi
$$

a condition that excludes the possibility of a clockwise rotation. We immediately obtain the single solution

$$
\psi=+\frac{\pi}{2}
$$

which satisfies the conditions established in (12.10) and (12.19), that is: the smallest rotation that goes from $\nabla \theta$ to $\nabla v$ is always a $\pi / 2$ angle, if $\theta$ is measured in the same direction (see also KozENY, loc. cit.).

Considering how we obtained conditions (12.10) and (12.19), it is easy to see that this theorem is valid for both stationary and non-stationary motion, because this result is simply a consequence of the kinematic conditions (12.3) and (12.4). The fbw must, however, be two-dimensional and irrotational for an incompressible fluid.

\section{References}

[1] ERTEL, H., Orthogonale Trajektoriensysteme in stationären ebenen Strömungsfeldern inkompressibler idealer Flüssigkeiten. In: Deutsche Akademie der Wissenschaften zu Berlin 1946-1956, Berlin 1956, 67-91.

[2] Kozeny, J., Hydraulik, Wien 1953, 292.

\section{ERTEL (1963a): Analogy between the equations of motion and the vortex equations in hydrodynamics}

\section{Summary}

An analogy between Euler's motion equations and Helmholtz's vortex equations can be found through two simple transformations.

\section{Notation and symbols}

Tensorial notation is used. All indices vary between 1 and 3; summations are made over the repeated index. $\epsilon_{i j k}$ is the alternating tensor (see, for example, F. MORÁn [4]). $x_{i}$ are the rectangular cartesian coordinates, $\mathbf{v}=\left\{v_{i}\right\}$ is the velocity vector $(|\mathbf{v}|=v), \boldsymbol{\xi}=$ $\left\{\xi_{i}\right\}$ the vorticity vector, $\Phi$ the external force potential, $p$ the pressure, $\rho$ the density, and $t$ the time.

\section{The problem}

We are accustomed to writing Euler's fundamental equations of hydrodynamics as

$$
\begin{aligned}
\frac{\partial v_{i}}{\partial t}+v_{j} \frac{\partial v_{i}}{\partial x_{j}} & =-\frac{\partial}{\partial x_{i}}\left(\Phi+\frac{p}{\rho}\right), \\
\frac{\partial v_{j}}{\partial x_{j}} & =0
\end{aligned}
$$

for a homogeneous and incompressible fluid. Taking the curl of (13.1), using (13.2) and substituting the expression for vorticity which is given by

$$
\xi_{i}=\epsilon_{i j k} \frac{\partial v_{k}}{\partial x_{j}},
$$

we obtain Helmholtz's famous vorticity equations

$$
\frac{\partial \xi_{i}}{\partial t}+v_{j} \frac{\partial \xi_{i}}{\partial x_{j}}=\xi_{j} \frac{\partial v_{i}}{\partial x_{j}},
$$

(see, for example, M. LUCINI [3]).

Understandably, the equations of motion (13.1) do not coincide with the equations of vorticity (13.4). However, it is possible to show that there is an analogy between equations (13.1) and (13.4). In order to achieve 
this analogy it is necessary to transform both systems (13.1) and (13.4). The demonstration follows.

\section{Representation of the analogy}

Adding the identities

$$
v_{j} \frac{\partial v_{j}}{\partial x_{i}}=\frac{\partial}{\partial x_{i}}\left(\frac{v^{2}}{2}\right)
$$

to equations (13.1) it is possible to express the motion equations in the following form:

$$
\frac{\partial v_{i}}{\partial t}+v_{j}\left(\frac{\partial v_{j}}{\partial x_{i}}+\frac{\partial v_{i}}{\partial x_{j}}\right)=\frac{\partial L}{\partial x_{i}}
$$

where

$$
L=\frac{v^{2}}{2}-\left(\Phi+\frac{p}{\rho}\right)
$$

is Lagrange's function. The symmetric tensor

$$
D_{i j}(\mathbf{v})=\frac{\partial v_{j}}{\partial x_{i}}+\frac{\partial v_{i}}{\partial x_{j}}
$$

measures the deformation of the velocity field.

Let us now consider the vorticity equations (13.4). Using the identities (see H. ERTEL [1]):

$$
\xi_{j} \frac{\partial v_{i}}{\partial x_{i}}=\xi_{j} \frac{\partial v_{j}}{\partial x_{i}}=\frac{\partial\left(\xi_{j} v_{j}\right)}{\partial x_{i}}-v_{j} \frac{\partial \xi_{j}}{\partial x_{i}},
$$

one can immediately write the vorticity equations (13.4) as follows:

$$
\frac{\partial \xi_{i}}{\partial t}+v_{j}\left(\frac{\partial \xi_{j}}{\partial x_{i}}+\frac{\partial \xi_{i}}{\partial x_{j}}\right)=\frac{\partial M}{\partial x_{i}}
$$

where $M$ is the scalar function (see, for example, $\mathrm{H}$. ERTEL and C.-G. Rossby [2], K. Oswatitsch [5])

$$
M=\xi_{j} v_{j} .
$$

The symmetric tensor

$$
D_{i j}(\boldsymbol{\xi})=\frac{\partial \xi_{j}}{\partial x_{i}}+\frac{\partial \xi_{i}}{\partial x_{j}}
$$

measures the deformation of the vorticity field.

The equations we just derived, namely equations (13.6) and (13.10), express the analogy we were searching for. In this analogy there is a perfect correspondence between the partial derivatives

$$
\frac{\partial v_{i}}{\partial t}, \frac{\partial v_{j}}{\partial x_{i}}, \frac{\partial v_{i}}{\partial x_{j}}, \frac{\partial L}{\partial x_{i}},
$$

which appear in the motion equations (13.6), and

$$
\frac{\partial \xi_{i}}{\partial t}, \frac{\partial \xi_{j}}{\partial x_{i}}, \frac{\partial \xi_{i}}{\partial x_{j}}, \frac{\partial M}{\partial x_{i}},
$$

which appear in the vorticity equations (13.10).

One can also obtain generalized equations for compressible fluids, if we assume that the density $\rho=\rho(p)$, as a function of pressure, contains identical parameters for all particles. In this case the analogy sought is obtained by substituting $p / \rho$ by $\int d p / \rho$, and $\xi_{i}$ and $\xi_{j}$ by $\xi_{i} / \rho$ and $\xi_{j} / \rho$, respectively, in the corresponding expressions.

\section{References}

[1] ERTel, H., Ein System von Identitäten und seine Anwendung zur Transformation von Wirbelgleichungen der Hydrodynamik. Mber. Dt. Akad. Wiss. Berlin 4 (1962), 292.

[2] Ertel, H., and C.-G. Rossby, A new Conservation-Theorem of Hydrodynamics. Geofi s. pura e appl. XIV (1949), Fasc. 3-4.

[3] Lucini, M., Teoria de la Mecánica y sus aplicaciones, Tercera edición, Barcelona 1959, 510 y 653.

[4] MoRÁn, F., Los tensores cartesianos rectangulares, Madrid 1954, 8.

[5] Oswatitsch, K., Physikalische Grundlagon der Strömungslehre. Handb. d. Physik, Bd. VIII/1, Berline/Göttingen/Heidelberg 1959, 38. 


\section{ERTEL (1963b): Relationships between differential operators from vector calculus and Lagrange's bracket, with hydrodynamic applications}

\section{Summary}

We will demonstrate the following theorem as a hydrodynamical application of a more general problem: The curl of the material derivative of the vorticity of rotating particles in an ideal and homogeneous fluid may be represented using Lagrange's bracket.

For the sake of brevity we make use of tensor notation. All indices go from 1 to 3 and index repetition indicates summation. Later on, we will see that it is useful to introduce the permutation tensor $\epsilon_{i j k}$ and the Kronecker $\delta_{i j}$ [see, for instance, LAWDEN $\left({ }^{2}\right)$, F. MORÁN $\left({ }^{3}\right)$, Th. Vogel $\left.\left({ }^{5}\right)\right]$.

We choose a rectangular cartesian coordinate system, with axes $x_{j}$ and introduce a system with six functions $R_{m}$ and $S_{m}$ of the $x_{j}$ and $t$ (=time) to form the symbols

$$
[i, j]=\frac{\partial R_{m}}{\partial x_{i}} \frac{\partial S_{m}}{\partial x_{j}}-\frac{\partial R_{m}}{\partial x_{j}} \frac{\partial S_{m}}{\partial x_{i}}=-[j, i] .
$$

These so-called Lagrange brackets are very important tools in Analytical Mechanics [see, for instance, D. Morgenstern and I. SzABó $\left({ }^{4}\right)$, E. T. WhitTAKer $\left.\left({ }^{6}\right)\right]$.

Suppose now that the system of six equations $R_{m}$ and $S_{m}$ is chosen such that the additional conditions

$$
\left(S_{1}, S_{2}, S_{3}\right)=\mathbf{S}=\text { solenoidal vector }
$$

and

$$
\left(R_{1}, R_{2}, R_{3}\right)=\mathbf{R}=\operatorname{rot} \mathbf{S}
$$

are met.

In this case (14.1) becomes

$$
[i, j]=\epsilon_{i j k} \operatorname{rot}_{k}\{(T \operatorname{rot}-\operatorname{rot} T) \mathbf{S}\}
$$

where $T$ is the scalar operator

$$
T=\mathbf{S} \cdot \nabla=S_{m} \frac{\partial}{\partial x_{m}} .
$$

The demonstration of (14.4) is easily done as follows. From the definition of operator $T$, applied to $\mathbf{S}$, we obtain

$$
T \mathbf{S}=\nabla\left\{\frac{1}{2}(\mathbf{S})^{2}\right\}-\mathbf{S} \times \mathbf{R} .
$$

Now, following a simple transformation from vectorial calculus, we obtain

$$
\operatorname{rot}(\mathbf{S} \times \mathbf{R})=(\mathbf{R} \cdot \nabla) \mathbf{S}-T \mathbf{R}
$$

as the divergence of a curl vanishes, and also, due to condition (14.2), $\operatorname{div} \mathbf{S}=0$, so that

$$
(\mathbf{R} \cdot \nabla) \mathbf{S}=(T \operatorname{rot}-\operatorname{rot} T) \mathbf{S} .
$$

In order to obtain Lagrange's bracket, we take the curl of (14.8), whose component on the $x_{k}$ axis is

$$
\begin{aligned}
\epsilon_{r s k} \frac{\partial}{\partial x_{r}}\left(\mathbf{R} \cdot \nabla_{s}\right) & \\
& =\operatorname{rot}_{k}\{(T \operatorname{rot}-\operatorname{rot} T) \mathbf{S}\} .
\end{aligned}
$$

Moreover, we obtain

$$
\mathbf{R} \cdot \nabla S_{s}=R_{m} \frac{\partial S_{s}}{\partial x_{m}}
$$

and its equivalent

$$
\mathbf{R} \cdot \nabla S_{s}=R_{m} \frac{\partial S_{m}}{\partial x_{s}},
$$

since the curl of $\mathbf{S}$ is defined as

$$
\frac{\partial S_{m}}{\partial x_{s}}-\frac{\partial S_{s}}{\partial x_{m}}=\epsilon_{s m n} \operatorname{rot}_{n} \mathbf{S}=\epsilon_{s m n} R_{n},
$$

and therefore

$$
R_{m}\left(\frac{\partial S_{m}}{\partial x_{s}}-\frac{\partial S_{s}}{\partial x_{m}}\right)=\epsilon_{s m n} R_{m} R_{n} \equiv 0,
$$

[see also: H. ERTEL $\left({ }^{1}\right)$ ].

Substituting the value of $\mathbf{R} \cdot \nabla S_{s}$, given by (14.11), into (14.9) yields

$$
\epsilon_{r s k} \frac{\partial R_{m}}{\partial x_{r}} \frac{\partial S_{m}}{\partial x_{s}}=\operatorname{rot}_{k}\{(T \operatorname{rot}-\operatorname{rot} T) \mathbf{S}\} .
$$

Applying the permutation tensor $\epsilon_{i j k}$ to (14.14) and then using

$$
\epsilon_{i j k} \epsilon_{r s k}=\delta_{i r} \delta_{j s}-\delta_{j r} \delta_{i s},
$$

we obtain

$$
\begin{aligned}
\frac{\partial R_{m}}{\partial x_{i}} \frac{\partial S_{m}}{\partial x_{j}} & -\frac{\partial R_{m}}{\partial x_{j}} \frac{\partial S_{m}}{\partial x_{i}} \\
& =\epsilon_{i j k} \operatorname{rot}_{k}\{(T \operatorname{rot}-\operatorname{rot} T) \mathbf{S}\}
\end{aligned}
$$

QED.

Let us now take a look at the rotating motion of a homogeneous, ideal liquid as a sample application of 
(14.4). In this case there is an acceleration potential $\Omega$; then

$$
\frac{d \mathbf{v}}{d t}=\frac{\partial \mathbf{v}}{\partial t}+(\mathbf{v} \cdot \nabla) \mathbf{v}=-\nabla \Omega,
$$

where $\mathbf{v}$ is the velocity vector, and

$$
\frac{d}{d t}=\frac{\partial}{\partial t}+\mathbf{v} \cdot \nabla
$$

is the material derivative.

Keeping in mind the quantities we have previously mentioned, and since $\mathbf{v}$ is a solenoidal vector, we can establish the following relationships:

$$
\begin{aligned}
\mathbf{S} & =\mathbf{v} \\
\mathbf{R} & =\nabla \times \mathbf{v}=\boldsymbol{\xi}=\text { vorticity, } \\
T & =\mathbf{v} \cdot \nabla=\frac{d}{d t}-\frac{\partial}{\partial t} .
\end{aligned}
$$

Moreover, in virtue of (14.17) and (14.21) we have

$$
\begin{gathered}
\nabla \times(T \mathbf{v})=-\frac{\partial \boldsymbol{\xi}}{\partial t} \\
(T \operatorname{rot}-\operatorname{rot} T) \mathbf{v}=\frac{d \boldsymbol{\xi}}{d t}
\end{gathered}
$$

Substituting this expression into equation (14.4), we have

$$
[i, j]=\epsilon_{i j k} \operatorname{rot}_{k}\left(\frac{d \boldsymbol{\xi}}{d t}\right)
$$

or, introducing the representation in terms of the components we have

$$
\left\{\begin{array}{l}
{[2,3]=\operatorname{rot}_{1}\left(\frac{d \boldsymbol{\xi}}{d t}\right),} \\
{[3,1]=\operatorname{rot}_{2}\left(\frac{d \boldsymbol{\xi}}{d t}\right),} \\
{[1,2]=\operatorname{rot}_{3}\left(\frac{d \boldsymbol{\xi}}{d t}\right),}
\end{array}\right.
$$

which demonstrates the theorem below.

The curl of the material derivative of the vorticity of rotating particles in a homogeneous and ideal fluid may be represented using Lagrange's brackets (non conservative).

This demonstration may also be done in a slightly different manner, by taking the appropriate transformation of the curl of Helmholtz's differential equation in vectorial notation. This, however, again requires use of the fundamental equalities in $(14.10,14.11)$.

\section{References}

(1) H. ERTEL: Ein System von Identitäten und seine Anwendung zur Transformation von Wirbelgleichungen der Hydrodynamik. Monatsberichte der Dt. Akad. Wiss. Berlin. Bd. 4, 1962, 292.

$\left(^{2}\right)$ D. F. LAWDEN: An Introduction to Tensor Calculus and Relativity. London-New York 1962, 23-37.

$\left.{ }^{3}\right)$ F. MORÁn: Los tensores cartesianos rectangulares. Madrid 1954, 1-9, 172.

$\left.{ }^{4}\right)$ D. Morgenstern and I. Szabó: Vorlesungen über Theoretische Mechanik. Berlin-Göttingen-Heidelberg 1961, 31, 40, 46.

$\left(^{5}\right)$ Th. Vogel: Physique mathématique classique. Paris 1956, 38.

$\left({ }^{6}\right)$ E. T. WhitTAKeR: A Treatise on the Analytical Dynamics of Particles and Rigid Bodies. Fourth Edition. Cambridge 1952, 298.

\section{ERTEL (1964): The maximum number of independent invariants in hydro- dynamics}

\section{Summary}

A theorem from the theory of partial differential equations allows the demonstration that the maximun number of independent invariants in hydrodynamics is equal to the number of dimensions in the reference space.

In order to study the general characteristics of the movement in any fluid it is more convenient to use orthogonal coordinates $x_{1}, x_{2} \ldots, x_{n}$ in a Euclidean space of $n$ dimensions. If we project the successive positions of the fluid particles along their trajectories during a period of time $t$ onto the coordinate axes, the $n$ dimensional velocity of the fluid particles will have the following $n$ components

$$
\left(v_{1}, v_{2}, \ldots, v_{n}\right)=\frac{d}{d t}\left(x_{1}, x_{2}, \ldots, x_{n}\right)
$$


where the operator

$$
\frac{d}{d t}=\frac{\partial}{\partial t}+v_{1} \frac{\partial}{\partial x_{1}}+v_{2} \frac{\partial}{\partial x_{2}}+\cdots+v_{n} \frac{\partial}{\partial x_{n}}
$$

indicates a derivative following the motion of the fluid.

Let $\psi=\psi\left(x_{1}, x_{2} \cdots, x_{n}, t\right)$ be a point function that defines a property of the hydrodynamic field during a period of time $t$. If this property is stationary on the moving particles

$$
\frac{d \psi}{d t}=\frac{\partial \psi}{\partial t}+v_{1} \frac{\partial \psi}{\partial x_{1}}+v_{2} \frac{\partial \psi}{\partial x_{2}}+\ldots+v_{n} \frac{\partial \psi}{\partial x_{n}}=0
$$

which means that $\psi$ remains constant if we move with one of the particles. When (15.3) is true, the variable $\psi$ is called a 'material invariant' or 'hydrodynamic invariant' with respect to the trajectory considered. The study of these hydrodynamic invariants is very important in dynamic meteorology and magnetohydrodynamics (see, for example, [1], [2], [3], [4], [6], [8]).

The identification of a given particle in an $n$ dimensional fluid along its trajectory during a period of time $t \geq 0$ requires knowledge of $n$ independent quantities which satisfy (15.3). As an example, we may use Lagrange's initial coordinates $\left\{a_{1}, a_{2} \ldots, a_{n}\right\}$ which form a completely independent set.

All of the initial coordinates satisfy (15.3), and therefore the system of parametric equations for their trajectory

$$
\left.\begin{array}{c}
x_{1}=x_{1}\left(a_{1}, a_{2} \ldots, a_{n}, t\right), \\
x_{2}=x_{2}\left(a_{1}, a_{2} \ldots, a_{n}, t\right), \\
\vdots \\
x_{n}=x_{n}\left(a_{1}, a_{2} \ldots, a_{n}, t\right),
\end{array}\right\}
$$

contains the $n$ hydrodynamic invariants $a_{1}, a_{2}, \ldots, a_{n}$ as solutions of Lagrange's fundamental hydrodynamic equations.

By virtue of the continuity equation, we know that the determinant of the transformation cannot vanish:

$$
\frac{\partial\left(x_{1}, x_{2}, \ldots, x_{n}\right)}{\partial\left(a_{1}, a_{2}, \ldots, a_{n}\right)} \neq 0 .
$$

Therefore, solving (15.4) for $a_{1}, a_{2}, \ldots, a_{n}$, one obtains

$$
\left.\begin{array}{c}
a_{1}=a_{1}\left(x_{1}, x_{2}, \ldots, x_{n}, t\right), \\
a_{2}=a_{2}\left(x_{1}, x_{2}, \ldots, x_{n}, t\right), \\
\vdots \\
a_{n}=a_{n}\left(x_{1}, x_{2}, \ldots, x_{n}, t\right) .
\end{array}\right\}
$$

Other hydrodynamic invariants can be added which correspond to certain thermodynamic conditions. An example of that is the entropy in adiabatic nondissipative fbws (see, for example, [9]).

One should, however, conjecture that more than $n$ hydrodynamic invariants will be functionally dependent. The demonstration of this hypothesis is very simple: Given $n$ invariants $a_{1}, a_{2}, \ldots, a_{n}$ and an additional different invariant $J$, the system of $n$ conditions

$$
\left.\begin{array}{c}
\left(\frac{\partial}{\partial t}+v_{1} \frac{\partial}{\partial x_{1}}+v_{2} \frac{\partial}{\partial x_{2}}+\cdots+v_{n} \frac{\partial}{\partial x_{n}}\right) a_{1}=0, \\
\left(\frac{\partial}{\partial t}+v_{1} \frac{\partial}{\partial x_{1}}+v_{2} \frac{\partial}{\partial x_{2}}+\cdots+v_{n} \frac{\partial}{\partial x_{n}}\right) a_{2}=0, \\
\vdots \\
\left(\frac{\partial}{\partial t}+v_{1} \frac{\partial}{\partial x_{1}}+v_{2} \frac{\partial}{\partial x_{2}}+\cdots+v_{n} \frac{\partial}{\partial x_{n}}\right) a_{n}=0,
\end{array}\right\}
$$

along with the additional condition

$$
\left(\frac{\partial}{\partial t}+v_{1} \frac{\partial}{\partial x_{1}}+v_{2} \frac{\partial}{\partial x_{2}}+\cdots+v_{n} \frac{\partial}{\partial x_{n}}\right) J=0
$$

constitutes a heterogeneous linear system of $n+$ 1 equations with $n$ unknowns $v_{1}, v_{2}, \ldots, v_{n}$ (where $J, a_{1}, a_{2}, \ldots, a_{n}$ and all of their first order partial derivatives are continuous functions). From algebraic analysis we know that a heterogeneous linear system of $n+1$ equations and $n$ unknowns will only admit non trivial solutions when the determinant formed by the coefficients and the independent terms vanishes (see, for example [7]).

The condition of compatibility of the system of equations comprised by (15.7) and (15.8) is

$$
\begin{aligned}
& \left|\begin{array}{ccccc}
\frac{\partial J}{\partial t} & \frac{\partial J}{\partial x_{1}} & \frac{\partial J}{\partial x_{2}} & \cdots & \frac{\partial J}{\partial x_{n}} \\
\frac{\partial a_{1}}{\partial t} & \frac{\partial a_{1}}{\partial x_{1}} & \frac{\partial a_{1}}{\partial x_{2}} & \cdots & \frac{\partial a_{1}}{\partial x_{n}} \\
\vdots & \vdots & \vdots & \cdots & \vdots \\
\frac{\partial a_{n}}{\partial t} & \frac{\partial a_{n}}{\partial x_{1}} & \frac{\partial a_{n}}{\partial x_{2}} & \cdots & \frac{\partial a_{n}}{\partial x_{n}}
\end{array}\right|=\frac{\partial\left(J, a_{1}, a_{2}, \ldots, a_{n}\right)}{\partial\left(t, x_{1}, x_{2}, \ldots, x_{n}\right)} \\
& =0 \text {. }
\end{aligned}
$$

This condition implies functional dependence between the functions $J, a_{1}, a_{2}, \ldots, a_{n}$ of the variables $x_{1}, x_{2}, \ldots, x_{n}$ :

$$
J=J\left(a_{1}, a_{2}, \ldots, a_{n}\right)
$$

(see, for example, [5]). It follows that the maximum number of independent hydrodynamic invariants 
is equal to the number of dimensions of the reference space, as we wanted to prove.

This result may be enunciated as the application of a theorem from the theory of partial differential equations, namely: Any function $\mathbf{J}$ which obeys (15.8) should verify (15.9) (eliminating $v_{1}, v_{2}, \ldots, v_{n}$, combined with (15.7)), which proves that there is a functional dependence between $J, a_{1}, a_{2}, \ldots, a_{n}$ (see, for example, [10]).

\section{References}

[1] ERTEL, H., Über Hydrodynamische Wirbelätze. Physikal. Z. 43 (1942), 526-520.

[2] ERTEL, H., Teorema sobre invariantes sustanciales de la Hidrodinámica. Gerl. Beitr. Geophys. 69 (1960), 290-293.

[3] Ertel, H., and C.-G. Rossby, A new Conservation-theorem of Hydrodynamics. Geofi s. pura y appl. XIV (1949), 189-193.
[4] FORTAK, H., Zur Frage allgemeiner hydrodynamischer Erhaltungssätze. Gerl. Beitr. Geophys. 65 (1956), 283-294.

[5] GillesPiE, R.P., Partial Differentiation, Edinburgh/London 1951, 43-46.

[6] HollmanN, G., Ein vollständiges System hydrodynamischer Erhaltungssätze. Arch. Meterol. Geophys. Bioklim. A 14 (1964). 1-13.

[7] Mataix Aracil, C., Análisis algébrico e infi nitesimal, Tomo I, Quinta edición Madrid 1957, 304-305.

[8] Mauersberger, P., Zur Kinematik der magnetischen Feldlinien im Plasma. Pure appl. Geophys. 87 (1964), I, 143-148.

[9] Móran Samaniego, F., Apuntes de Termodinámica de la Atmósfera, Madrid 1944, 70-81.

[10] PUig AdAm, P., Ecuaciones diferenciales aplicadas a la Física y Teénica, Madrid 1958, 214-215.

\section{ERTEL (1965a): Hydrodynamic commutation formulas}

\section{Summary}

From the Lagrangian form of the hydrodynamic equations for perfect fluids a commutation relation is derived. The continuity equation is not necessary for this derivation. This commutation relation, expressed in Lagrangian arguments, can be transformed by additional consideration of the continuity equation into a commutation relation expressed in Eulerian arguments.

\section{Symbols and fundamental equations}

In the following we will use the symbols of tensor analysis in three-dimensional Euclidean space. All indices range over the values $1,2,3$. In a term with repeated indices we use the summation convention, with the sum taken from 1 to 3 .

Define

$$
\delta_{i j}=\left\{\begin{aligned}
+1 & \text { if } i=j, \\
0 & \text { if } i \neq j,
\end{aligned}\right.
$$

as the substitution tensor and

$$
\epsilon_{i j k}=\left\{\begin{aligned}
+1, & \text { if } i, j, k \text { form a cyclic sequence } \\
-1, & \text { if } i, j, k \text { form a noncyclic sequence } \\
0, & \text { if any two indices agree }
\end{aligned}\right.
$$

as the permutation symbol.
The hydrodynamic equations for a perfect gas can be written in the Lagrangian form

$$
\begin{aligned}
& \frac{\partial^{2} x_{m}}{\partial t^{2}} \frac{\partial x_{m}}{\partial a_{j}}=-\frac{\partial \Phi}{\partial a_{j}}-\sigma \frac{\partial p}{\partial a_{j}} \quad \text { (momentum equation) } \\
& \sigma_{0}=\sigma D^{-1}
\end{aligned}
$$

(continuity equation)

or in the Eulerian form

$$
\begin{aligned}
\frac{d v_{j}}{d t}=\left(\frac{\partial}{\partial t}+v_{k} \frac{\partial}{\partial x_{k}}\right) v_{j}= & -\frac{\partial \Phi}{\partial x_{j}}-\sigma \frac{\partial p}{\partial x_{j}} \\
& (\text { momentum equation) } \\
\frac{d \sigma}{d t}= & \sigma \frac{\partial v_{k}}{\partial x_{k}} \\
& \text { (continuity equation) }
\end{aligned}
$$

where we have defined the scalars $\Phi=$ potential for external forces, $p=$ pressure, $\sigma=$ specific volume (reciprocal of the density), $t=$ time, and in (16.2) the functional determinant

$$
D=\frac{\partial\left(x_{1}, x_{2}, x_{3}\right)}{\partial\left(a_{1}, a_{2}, a_{3}\right)}
$$


of the position coordinates $x_{j}=x_{j}\left(a_{1}, a_{2}, a_{3}\right)$ of a fluid particle for $t \geq 0$ with respect to the labeling coordinates $a_{j}$, and the specific volume $\sigma_{0}=\sigma_{0}\left(a_{1}, a_{2}, a_{3}\right)$ at $t=0$.

In the Lagrangian framework (16.1) and (16.2) the independent variables are the labeling coordinates $a_{j}$ and the time $t$, while in the Eulerian framework (16.3) and (16.4) the independent variables are the orthogonal cartesian coordinates $x_{j}$ and the time $t$. The material derivative in the Lagrangian framework is denoted by the operator $\partial / \partial t$, while in the Eulerian framework by the operator $d / d t=\partial / \partial t+v_{k}\left(\partial / \partial x_{k}\right)$, so that, for example, the wind components in the Lagrangian framework are given by

$$
\frac{\partial}{\partial t} x_{j}\left(a_{1}, a_{2}, a_{3}, t\right)=\frac{\partial x_{j}}{\partial t}=v_{j}
$$

and in the Eulerian framework by

$$
\begin{aligned}
\frac{d x_{j}}{d t}=\left(\frac{\partial}{\partial t}+v_{k} \frac{\partial}{\partial x_{k}}\right) x_{j} & =v_{k} \frac{\partial x_{j}}{\partial x_{k}} \\
& =v_{k} \delta_{j k}=v_{j} .
\end{aligned}
$$

The scalar fields $p, \sigma, \Phi$ in the Lagrangian framework are functions of the independent variables $a_{j}, t$ and in the Eulerian framework are functions of the independent variables $x_{j}, t$.

\section{Derivation of a commutation relation from the La- grangian form of the hydrodynamical equations}

We proceed from the Lagrangian momentum equations (16.1), which, in consideration of (16.6), can be written as

$$
\frac{\partial v_{m}}{\partial t} \frac{\partial x_{m}}{\partial a_{j}}=-\frac{\partial \Phi}{\partial a_{j}}-\sigma \frac{\partial p}{\partial a_{j}} .
$$

We then apply to (16.8) the differential operator $\epsilon_{i j k} \frac{\partial \psi}{\partial a_{k}} \frac{\partial}{\partial a_{i}}$, containing the differentiable function $\psi$. Considering the antisymmetry of $\epsilon_{i j k}$, we have:

$$
\begin{aligned}
\epsilon_{i j k} \frac{\partial \psi}{\partial a_{k}} \frac{\partial^{2} v_{m}}{\partial t \partial a_{i}} \frac{\partial x_{m}}{\partial a_{j}} & =-\epsilon_{i j k} \frac{\partial \sigma}{\partial a_{i}} \frac{\partial p}{\partial a_{j}} \frac{\partial \psi}{\partial a_{k}} \\
& =+\epsilon_{i j k} \frac{\partial p}{\partial a_{i}} \frac{\partial \sigma}{\partial a_{j}} \frac{\partial \psi}{\partial a_{k}}
\end{aligned}
$$

or, again considering (16.6) and $\epsilon_{i j k} \frac{\partial v_{m}}{\partial a_{i}} \frac{\partial v_{m}}{\partial a_{j}}=0$ :

$$
\begin{array}{r}
\frac{\partial}{\partial t}\left(\epsilon_{i j k} \frac{\partial v_{m}}{\partial a_{i}} \frac{\partial x_{m}}{\partial a_{j}} \frac{\partial \psi}{\partial a_{k}}\right) \\
-\left(\epsilon_{i j k} \frac{\partial v_{m}}{\partial a_{i}} \frac{\partial x_{m}}{\partial a_{j}} \frac{\partial}{\partial a_{k}}\right) \frac{\partial \psi}{\partial t} \\
=\epsilon_{i j k} \frac{\partial p}{\partial a_{i}} \frac{\partial \sigma}{\partial a_{j}} \frac{\partial \psi}{\partial a_{k}} .
\end{array}
$$

The right hand side of (16.10) is the functional determinant of the functions $p, \sigma, \psi$ with respect to the coordinates $a_{1}, a_{2}, a_{3}$, i.e.,

$$
\epsilon_{i j k} \frac{\partial p}{\partial a_{i}} \frac{\partial \sigma}{\partial a_{j}} \frac{\partial \psi}{\partial a_{k}}=\frac{\partial(p, \sigma, \psi)}{\partial\left(a_{1}, a_{2}, a_{3}\right)} .
$$

The two terms of the left side of (16.10) contain the Lagrangian bracket symbols from analytical mechanics (see, for example, E. T. WHITTAKER [16]), here in (16.10) for the quantities $v_{m}$ and $x_{m}(m=1,2,3)$ :

$$
\epsilon_{i j k} \frac{\partial v_{m}}{\partial a_{i}} \frac{\partial x_{m}}{\partial a_{j}}=\{v, x\}_{k}
$$

where

$$
\left.\begin{array}{rl}
v & \equiv v_{m}\left(a_{1}, a_{2}, a_{3}, t\right) \\
\text { and } & \\
x & \equiv x_{m}\left(a_{1}, a_{2}, a_{3}, t\right)
\end{array}\right\}
$$

are used as abbreviations for the wind components and the position coordinates.

The substitution of (16.12) into (16.10) results in the sought-after commutation relation in the form:

$$
\begin{aligned}
\left(\frac{\partial}{\partial t}\right)\left(\{v, x\}_{k} \frac{\partial}{\partial a_{k}}\right) \psi & -\left(\{v, x\}_{k} \frac{\partial}{\partial a_{k}}\right)\left(\frac{\partial}{\partial t}\right) \psi \\
& =\epsilon_{i j k} \frac{\partial p}{\partial a_{i}} \frac{\partial \sigma}{\partial a_{j}} \frac{\partial \psi}{\partial a_{k}}
\end{aligned}
$$

or, written in operator form:

$$
\begin{aligned}
\left(\frac{\partial}{\partial t}\right)\left(\{v, x\}_{k} \frac{\partial}{\partial a_{k}}\right) & -\left(\{v, x\}_{k} \frac{\partial}{\partial a_{k}}\right)\left(\frac{\partial}{\partial t}\right) \\
& =\epsilon_{i j k} \frac{\partial p}{\partial a_{i}} \frac{\partial \sigma}{\partial a_{j}} \frac{\partial}{\partial a_{k}}
\end{aligned}
$$

These commutation relations, (16.14) and (16.15), state that the operators

$$
\frac{\partial}{\partial t} \quad \text { and } \quad\{v, x\}_{k} \frac{\partial}{\partial a_{k}}
$$

commute when applied to any (twice differentiable) function $\psi$ if there exists for all flid particles the same piezotropic relation

$$
\sigma=\sigma(p)
$$

If the relationship (16.17) does not exist, the operators (16.16), when applied to a function of the special 
form $\psi=\psi(p, \sigma)$, also commute, since in this case the functional determinant (16.11) likewise disappears. It is natural that the commutation property of the operators (16.16) always applies for homogeneous ( $\sigma=$ const) perfect fluids, since constant $\sigma$ makes the right sides of (16.14) and (16.15) vanish.

The commutation relations (16.14) and (16.15) make possible the derivation of conservation laws. Consider a flid for which there exists one piezotropic relation of the form (16.17). We can select for $\psi$, for example, each labeling coordinate $a_{j}$. Since

$$
\frac{\partial a_{j}}{\partial t}=0
$$

always holds, (16.14) reduces to

$$
\begin{aligned}
\left(\frac{\partial}{\partial t}\right)\left(\{v, x\}_{k} \frac{\partial a_{j}}{\partial a_{k}}\right) & =\left(\frac{\partial}{\partial t}\right)\left(\{v, x\}_{k} \delta_{j k}\right) \\
& =\left(\frac{\partial}{\partial t}\right)\left(\{v, x\}_{j}\right)=0,
\end{aligned}
$$

which says: The Lagrangian bracket symbols are in this case constant along the trajectory of each individual particle, a result that finds multiple applications in classical hydrodynamics (see, for example, H. LAMB [10]).

\section{Derivation of a commutation relation from the Eu- lerian form of the hydrodynamical equations}

Starting from the Eulerian form of the momentum equations for perfect fluids (16.3) and the continuity equation (16.4), the following commutation relation can be derived (H. ERTEL [1,2]; D. Morgenstern and I. SZABó [13], K. OSWATITSCH [14]):

$$
\begin{aligned}
\left(\frac{d}{d t}\right)\left(\sigma \xi_{k} \frac{\partial}{\partial x_{k}}\right) \psi & -\left(\sigma \xi_{k} \frac{\partial}{\partial x_{k}}\right)\left(\frac{d}{d t}\right) \psi \\
& =\sigma \epsilon_{i j k} \frac{\partial p}{\partial x_{i}} \frac{\partial \sigma}{\partial x_{j}} \frac{\partial \psi}{\partial x_{k}}
\end{aligned}
$$

where

$$
\xi_{k}=\epsilon_{i j k} \frac{\partial v_{j}}{\partial x_{i}}
$$

are the components of the vorticity vector (curl of the velocity vector). This commutation relation (16.20), expressed in the Eulerian framework, has already been repeatedly used in special and generalized applications (H. Ertel [3,4], H. Fortak [6], G. Hollmann [7], P. MAUERSBERGER [12]).

\section{The relation between the two commutation for- mulas}

Since the derivation of (16.20) requires consideration of the continuity equation, an equation that was not necessary for the derivation of the commutation relation (16.14), the transformation of (16.14) to (16.20) and vice versa is of interest for discovering the connection between the two commutation relations (16.14) and (16.20).

In order to transform for example (16.14) into (16.20), one takes the product of (16.14) and (16.2), an operation which is equivalent to the introduction of the continuity equation (16.2) into (16.14). With consideration of the time-independence of $\sigma_{0}$, we obtain:

$$
\begin{gathered}
\left(\frac{\partial}{\partial t}\right)\left(\sigma_{0}\{v, x\}_{k} \frac{\partial}{\partial a_{k}}\right) \psi \\
-\left(\sigma_{0}\{v, x\}_{k} \frac{\partial}{\partial a_{k}}\right)\left(\frac{\partial}{\partial t}\right) \psi \\
=\sigma D^{-1} \epsilon_{i j k} \frac{\partial p}{\partial a_{i}} \frac{\partial \sigma}{\partial a_{j}} \frac{\partial \psi}{\partial a_{k}},
\end{gathered}
$$

the right side of which can be written, using (16.5) and (16.11), as

$$
\begin{aligned}
\sigma D^{-1} & \epsilon_{i j k} \frac{\partial p}{\partial a_{i}} \frac{\partial \sigma}{\partial a_{j}} \frac{\partial \psi}{\partial a_{k}} \\
& =\sigma \frac{\partial(p, \sigma, \psi)}{\partial\left(a_{1}, a_{2}, a_{3}\right)}\left(\frac{\partial\left(x_{1}, x_{2}, x_{3}\right)}{\partial\left(a_{1}, a_{2}, a_{3}\right)}\right)^{-1}
\end{aligned}
$$

which in turn, with the use of the well-known functional determinant product rule, becomes

$$
\begin{aligned}
\sigma D^{-1} \epsilon_{i j k} \frac{\partial p}{\partial a_{i}} \frac{\partial \sigma}{\partial a_{j}} \frac{\partial \psi}{\partial a_{k}} & =\sigma \frac{\partial(p, \sigma, \psi)}{\partial\left(x_{1}, x_{2}, x_{3}\right)} \\
& =\sigma \epsilon_{i j k} \frac{\partial p}{\partial x_{i}} \frac{\partial \sigma}{\partial x_{j}} \frac{\partial \psi}{\partial x_{k}}
\end{aligned}
$$

which is in agreement with the right side of (16.20).

On the left side of (16.20) the following rearrangement is possible:

$$
\begin{aligned}
&\{v, x\}_{k} \frac{\partial}{\partial a_{k}}=\epsilon_{i j k} \frac{\partial v_{m}}{\partial a_{i}} \frac{\partial x_{m}}{\partial a_{j}} \frac{\partial}{\partial a_{k}} \\
&=\epsilon_{i j k} \frac{\partial x_{q}}{\partial a_{i}} \frac{\partial v_{m}}{\partial x_{q}} \cdot \frac{\partial x_{r}}{\partial a_{j}} \frac{\partial x_{m}}{\partial x_{r}} \cdot \frac{\partial x_{s}}{\partial a_{k}} \frac{\partial}{\partial x_{s}} \\
&=\epsilon_{i j k} \frac{\partial x_{q}}{\partial a_{i}} \frac{\partial x_{r}}{\partial a_{j}} \frac{\partial x_{s}}{\partial a_{k}} \cdot \frac{\partial v_{m}}{\partial x_{q}} \frac{\partial x_{m}}{\partial x_{r}} \frac{\partial}{\partial x_{s}} .
\end{aligned}
$$

Using the general formula of the determinant symbolism (see, for example, H. JEFFREYs [8], H. JEFFREYS and B. SWIRLES [9], A. J. MCCONNELl [11], G. TEMPLE [15])

$$
\epsilon_{i j k} \frac{\partial x_{q}}{\partial a_{i}} \frac{\partial x_{r}}{\partial a_{j}} \frac{\partial x_{s}}{\partial a_{k}}=\epsilon_{q r s} D,
$$


and the substitution tensor

$$
\frac{\partial x_{m}}{\partial x_{r}}=\delta_{m r}
$$

in (16.25), we obtain, with consideration of (16.26), (16.21) and (16.2), the operator equation:

$$
\begin{aligned}
\sigma_{0}\{v, x\}_{k} \frac{\partial}{\partial a_{k}} & =\sigma_{0} D \epsilon_{q r s} \frac{\partial v_{r}}{\partial x_{q}} \frac{\partial}{\partial x_{s}} \\
& =\sigma \xi_{s} \frac{\partial}{\partial x_{s}}=\sigma \xi_{k} \frac{\partial}{\partial x_{k}} .
\end{aligned}
$$

The substitution of (16.24) and (16.28) in (16.22) and the change from the Lagrangian to the Eulerian form of the material derivative $\partial / \partial t \rightarrow d / d t$ yields the commutation relation (16.20), q.e.d.

The inverse transformation, that is the elimination of the continuity equation from (16.20) to recover the commutation relation (16.14), has already discussed in another paper (H. Ertel [5]). Thus, using the continuity equation (16.2) with the functional determinant (16.5), a reversible connection between the two commutation relations (16.14) and (16.20) has been established. The transformation of the commutation relations (16.14) and (16.20) into one another is always executable, since the continuity equation, as an expression of the law of the conservation of fluid matter, provides a guarantee for it in that the functional determinant (16.5) is always different from zero.

\section{References}

[1] ERTEL, H., Über hydrodynamische Wirbelsätze. Physikal. Zeitschr., Leipzig, 43 Jahrg. (1942) 526-529.

[2] ERTEL, H., Ein neuer hydrodynamischer Wirbelsatz. Meteorol. Zeitschr., Braunschweig, 59. Jahrg. (1942) 277-281.

[3] ERTEL, H., Über das Verhältnis des neuen hydrodynamischen Wirbelsatzes zum Zirkulationsatz von V. BJERKNES. Meteorol. Zeitschr., Braunschweig, 59. Jahrg. (1942) 385-387.
[4] ERTEL, H., Ein neuer hydrodynamischer Erhaltungssatz. Die Naturwissenschaften, Berlin, 30. Jahrg. (1942) 543-544.

[5] ERTEL, H., Vertauschungs-Relationen der Hydrodynamik. Monatsber. Dt. Akad. Wiss., Berlin, Bd. 6 (1964) 838-841.

[6] Fortak, H., Zur Frage allgemeiner hydrodynamischer Erhaltungssätze. Gerlands Beitr. z. Geophysik, Leipzig, Bd. 65 (1956) 283-294.

[7] HollmanN, G., Ein vollständiges System hydrodynamischer Erhaltungssätze. Archiv f. Meteorologie, Geophysik u. Bioklimatologie, Wien, Ser. A, Bd. 14 (1964) 1-13.

[8] Jeffreys, H., Cartesian Tensors. Cambridge 1952 (Reprinted), 15.

[9] JefFreys, H., and B. Swirles, Methods of Mathematical Physics. Third Edition. Cambridge 1956, 72.

[10] Lamb, H., Hydrodynamics. First American Edition. New York 1945, 204.

[11] McConnell, A. J., Applications of the Absolute Differential Calculus. London and Glasgow 1951 (Reprinted), 10-11.

[12] Mauersberger, P., Zur Kinematik der magnetischen Feldlinien im Plasma. Pure and Applied Geophysics, Basel, Vol. 87 (1964/I) 143-148.

[13] Morgenstern, D., and I. Szabó, Vorlesungen über Theoretische Mechanik. Berlin-Göttingen-Heidelberg 1961, 182.

[14] Oswatitsch, K., Physikalische Grundlagen der Strömungslehre. In: Handbuch der Physik, herausg. von S. FLÜGGE. Bd. VIII/1. Berlin-Göttingen-Heidelberg 1959, 43-45.

[15] Temple, G., Cartesian Tensors. London-New York $1960,18$.

[16] Whittaker, E. F., A Treatise on the Analytical Dynamics of Particles and Rigid Bodies. Fourth Edition. Cambridge 1952, 298. 


\section{ERTEL (1965b): Commutative operators for unsteady fbw of a perfect, piezotropic fhid}

The fbw of a perfect, piezotropic fluid (in the notation of, for example, J. SERRIN [7]) is governed by the momentum equation

$$
\frac{d v_{i}}{d t}=-\frac{\partial}{\partial x_{i}}\left(\Phi+\int \sigma d p\right),
$$

or equivalently,

$$
\frac{\partial v_{i}}{\partial t}=\epsilon_{i j k} v_{j} \xi_{k}-\frac{\partial H}{\partial x_{i}},
$$

by the continuity equation

$$
\frac{d \sigma}{d t}=\sigma \frac{\partial v_{j}}{\partial x_{j}}
$$

and by the piezotropic relation

$$
\sigma=\sigma(p),
$$

where $x_{i}(i=1,2,3)=$ orthogonal cartesian coordinates (right-handed system), $v_{i}(i=1,2,3)=$ components of the velocity vector $\mathbf{v}, \epsilon_{i j k}(i, j, k=1,2,3)$ $=$ permutation symbol (antisymmetric in all indices), and $\xi_{k}=\epsilon_{i j k} \frac{\partial v_{j}}{\partial x_{i}}=$ components of the vorticity vector $\boldsymbol{\xi}=\nabla \times \mathbf{v}$. The scalars $\Phi, \sigma, p, H=\frac{1}{2} v^{2}+\Phi+\int \sigma d p$ (with $v^{2}=v_{j} v_{j}$ ) and $t$ respectively denote the potential for external forces, the specific volume (reciprocal of density), the pressure, the energy, and the time; in addition

$$
\frac{d}{d t}=\frac{\partial}{\partial t}+v_{j} \frac{\partial}{\partial x_{j}}
$$

is the material differential operator of hydrodynamics. In the previous equations we consistly use the usual formulation of vector analysis (see, for example, G. H. A. COLE [1], J. O. HINZE [5]) with the Einstein summation convention. From equations (17.1), or equivalently (17.2), and (17.3), we deduce that the operators

$$
\frac{d}{d t} \quad \text { and } \quad \sigma \xi_{i} \frac{\partial}{\partial x_{i}} \quad(=\sigma \boldsymbol{\xi} \cdot \nabla)
$$

applied to the twice differentiable function $\psi=$ $\psi\left(x_{1}, x_{2}, x_{3}, t\right)$ are commutative (H. ERTEL [2], [3]; see, for example, D. Morgenstern and I. SzAbó [6]), so that

$$
\left(\frac{d}{d t}\right)\left(\sigma \xi_{i} \frac{\partial}{\partial x_{i}}\right) \psi-\left(\sigma \xi_{i} \frac{\partial}{\partial x_{i}}\right)\left(\frac{d}{d t}\right) \psi=0 .
$$

We wish to utilize the commutation relation (17.6) to prove that the operators

$$
\frac{d}{d t} \quad \text { and } \quad \sigma \xi_{i} \frac{\partial}{\partial t} \quad\left(=\sigma \boldsymbol{\xi} \frac{\partial}{\partial t}\right)
$$

are commutative when applied to the components of the flow velocity vector

$$
\mathbf{v}=\left\{v_{i}\right\} \quad(i=1,2,3)
$$

for the flow field of a perfect, piezotropic fluid:

$$
\left(\frac{d}{d t}\right)\left(\sigma \xi_{i} \frac{\partial}{\partial t}\right) v_{i}-\left(\sigma \xi_{i} \frac{\partial}{\partial t}\right)\left(\frac{d}{d t}\right) v_{i}=0
$$

or in the coordinate-independent formulation of vector analysis:

$$
\left(\frac{d}{d t}\right)\left(\sigma \boldsymbol{\xi} \frac{\partial}{\partial t}\right) \mathbf{v}-\left(\sigma \boldsymbol{\xi} \frac{\partial}{\partial t}\right)\left(\frac{d}{d t}\right) \mathbf{v}=0 .
$$

To prove (17.7), or equivalently (17.8), we set $\psi=H$ in (17.6), which results in

$$
\left(\frac{d}{d t}\right)\left(\sigma \xi_{i} \frac{\partial}{\partial x_{i}}\right) H-\left(\sigma \xi_{i} \frac{\partial}{\partial x_{i}}\right)\left(\frac{d}{d t}\right) H=0 .
$$

From the momentum equation in the form (17.2) it follows that

$$
\left(\sigma \xi_{i} \frac{\partial}{\partial x_{i}}\right) H=-\left(\sigma \xi_{i} \frac{\partial}{\partial t}\right) v_{i}
$$

whereas from the momentum equation in the form (17.1) it follows that

$$
\frac{d}{d t}\left(\frac{v^{2}}{2}\right)=-\left(v_{i} \frac{\partial}{\partial x_{i}}\right)\left(\Phi+\int \sigma d p\right)
$$

and, with consideration of (17.5) and the definition of the total energy $H$, that:

$$
\frac{d H}{d t}=\frac{\partial}{\partial t}\left(\Phi+\int \sigma d p\right)
$$

From this it immediately follows that

$$
\begin{aligned}
\left(\sigma \xi_{i} \frac{\partial}{\partial x_{i}}\right) & \left(\frac{d}{d t}\right) H \\
= & \left(\sigma \xi_{i} \frac{\partial}{\partial t}\right) \frac{\partial}{\partial x_{i}}\left(\Phi+\int \sigma d p\right),
\end{aligned}
$$


or with the use of (17.1):

$$
\left(\sigma \xi_{i} \frac{\partial}{\partial x_{i}}\right)\left(\frac{d}{d t}\right) H=-\left(\sigma \xi_{i} \frac{\partial}{\partial t}\right)\left(\frac{d}{d t}\right) v_{i}
$$

Substitution of (17.10) and (17.14) into (17.9) yields the desired equation (17.7), thereby completing our argument and demonstrating the coordinate-free notation of equation (17.8). It is possible to obtain equations (17.7) and (17.8) by another method, based on the hydrodynamical vorticity equation, in which yet another operation can be given, with the application to the fbw field of a perfect, piezotropic fluid yielding the commutation relation if the field is stationary (H. ERTEL [4]).

\section{References}

[1] Cole, G. H. A., Fluid Dynamics. London-New York, 1962, 3.

[2] H. ERTEL, Über hydrodynamische Wirbelsätze. Phys. Z., Leipzig, 43. Jahrg., 526 (1942).
[3] H. ERTEL, Hydrodynamische Vertauschungs-Relationen. Acta Hydrophysica, Berlin, Bd. IX, 115 (1965).

[4] H. ERTEL, Kommutative Operatoren der Geschwindigkeitsfelder perfekter, piezotroper Flüssigkeiten. Acta Hydrophysica, Berlin, Bd. X, Heft 2 (1965); z. Z. im Druck.

[5] Hinze, J. O., Turbulence. New York-Toronto-London 1959, 15.

[6] Morgenstern, D., and I. Szabó, Vorlesungen über Theoretische Mechanik. Berlin-Göttingen-Heidelberg 1961, 182.

[7] SERrin, J., Mathematical Principles of Classical Fluid Mechanics. In: Handbuch der Physik, herausgegeben von S. FLÜGGE. Band VIII/1. Berlin-Göttingen-Heidelberg 1959, 150.

\section{ERTEL (1965c): Theorem on unimodular transformations of the hydrody- namical labeling coordinates}

\section{Summary}

From the equations which express the dependence of the initial coordinates upon the coordinates of the space-time continuum we can form an invariant with respect to unimodular transformations from one system of initial coordinates to another.

\section{Introduction}

Assume that a flid fbws through an $n$-dimensional space. A particular point in space is given by the coordinates $\left\{x_{1}, x_{2}, \ldots, x_{n}\right\}$, measured with respect to a fixed frame of reference, and a particular particle is identified by the parameters $\left\{a_{1}, a_{2}, \ldots, a_{n}\right\}$ ("labeling coordinates," see, for example, V. BJERKNES, J. BJERKNes, H. Solberg, T. Bergeron [1], H. L. Dryden, F. D. Murnaghan, H. Bateman [2]). An example of labeling coordinates are the particle positions at $t=0$ ("Anfangs-coordinates"). Special presuppositions on the nature of the fluid (whether ideal or viscous, incompressible or compressible) are not necessary for our purposes.

For brevity the notation of tensor analysis is adopted. All indices range from 1 to $n$; a term with repeated indices is to be summed from 1 to $n$. Other symbols are defined in the text.

\section{Statement of the theorem}

The solutions of the hydrodynamical equations, including the required physical connections, are embodied in the system of implicit functions $(j=1,2, \ldots, n)$ :

$$
R_{j}\left(x_{1}, x_{2}, \ldots, x_{n}, a_{1}, a_{2}, \ldots, a_{n}, t\right)=0 .
$$

Solving for $x_{j}$ gives the parameter description of particle trajectories:

$$
x_{j}=x_{j}\left(a_{1}, a_{2}, \ldots, a_{n}, t\right),
$$

whereas solution for $a_{j}$ results in:

$$
a_{j}=a_{j}\left(x_{1}, x_{2}, \ldots, x_{n}, t\right),
$$

from which, for example for the purposes of meteorology, one can answer the question of which particle (air mass) passes through the fixed spatial coordinates $\left\{x_{1}, x_{2}, \ldots, x_{n}\right\}$ at time $t$.

In that the functions (18.2) are to be regarded as continuous and differentiable, and furthermore that the continuity equation in Lagrangian form requires that the functional determinant of $x_{1}, x_{2}, \ldots, x_{n}, t$ with respect to $a_{1}, a_{2}, \ldots, a_{n}, t$, or its inverse, is always different than zero, the system (18.2) is the inverse transformation of (18.2) (and vice versa). 
A system of infinitesimal variations $\delta x_{1}, \delta x_{2}, \ldots$, $\delta x_{n}, \delta t$ of the coordinates of space-time $x_{1}, x_{2}, \ldots$, $x_{n}, t$ is related to the variations of the labeling coordinates by

$$
\delta a_{j}=\frac{\partial a_{j}}{\partial x_{k}} \delta x_{k}+\frac{\partial a_{j}}{\partial t} \delta t \quad(j, k=1,2, \ldots, n) .
$$

Here the $\delta x_{k}(k=1,2, \ldots, n)$ and $\delta t$ are independently and freely selectable. The operator $\partial / \partial t$ denotes the local time derivative in the Eulerian sense, i.e.,

$$
\frac{\partial}{\partial t}=\left(\frac{\partial}{\partial t}\right)_{x_{1}, x_{2}, \ldots, x_{n}}
$$

not the operator for individual or material differentiation with respect to time:

$$
\frac{d}{d t}=\left(\frac{\partial}{\partial t}\right)_{a_{1}, a_{2}, \ldots, a_{n}}
$$

which makes possible simple expressions for preservation of the particle-bound properties (H. ERTEL [3], G. HollmanN [4]). For example, the individual preservation of the labeling coordinates is expressed by the system

$$
\frac{d a_{j}}{d t}=0 \quad(j=1,2, \ldots, n)
$$

while $\partial a_{j} / \partial t$ for a moving fluid can never disappear because of the local particle change at fixed spatial points.

According to (18.3), the partial derivatives $\partial a_{j} / \partial x_{k}$ and $\partial a_{j} / \partial t$ in (18.4) are functions of $x_{1}, x_{2}, \ldots, x_{n}$ and $t$. However, by means of (18.2), they can also be represented as functions of $a_{1}, a_{2}, \ldots, a_{n}$ and $t$. This latter representation is assumed for the purpose of the following calculations.

In place of the system $a_{j}(j=1,2, \ldots, n)$ we now transform to another system $b_{j}(j=1,2, \ldots, n)$ of labeling coordinates through the transformation

$$
b_{j}=b_{j}\left(a_{1}, a_{2}, \ldots, a_{n}\right) \quad(j=1,2, \ldots, n)
$$

with the transformation determinant

$$
\frac{\partial\left(b_{1}, b_{2}, \ldots, b_{n}\right)}{\partial\left(a_{1}, a_{2}, \ldots, a_{n}\right)}=1
$$

(unimodular transformation; see, for example, B. J. NAAS and H. L. Schmid [6], H. Reichardt [7], P. A. Schirokow and A. P. Schirokow [8], K. Strubecker [9], E. Vidal Abascal [10]). Then from (18.3) and (18.4), with the aid of (18.8) and (18.9), we deduce the following theorem:

The form

$$
F=\frac{\partial \delta a_{j}}{\partial a_{j}}
$$

is an invariant with respect to a unimodular transformation from one system of labeling coordinates to another.

\section{Proof of the theorem}

The variations (18.4) are related through (18.8) to the variations

$$
\delta b_{j}=b_{j, k} \delta a_{k}
$$

of the new labeling coordinates, where $\partial b_{j} / \partial a_{k}=b_{j, k}$.

Identifying $b_{j m}$ (without the comma between the indices) as the subdeterminant corresponding to the element $b_{j, m}$ of the determinant (18.9), the determinant adopts the form:

$$
b_{j m} b_{k, m}=b_{m j} b_{m, k}=\left\{\begin{aligned}
+1, & \text { if } j=k \\
0, & \text { if } j \neq k
\end{aligned}\right.
$$

Then it can be shown that the solutions of the system (18.11) for $\delta a_{j}$ can be written as

$$
\delta a_{j}=b_{k j} \delta b_{k},
$$

from which the expression in the above theorem can be written as

$$
\frac{\partial \delta a_{j}}{\partial a_{j}}=b_{k j, j} \delta b_{k}+b_{k j} \frac{\partial \delta b_{k}}{\partial a_{j}} .
$$

Through alteration of the summation indices on the right hand side, we obtain

$$
\frac{\partial \delta a_{j}}{\partial a_{j}}=b_{j k, k} \delta b_{j}+b_{j m} \frac{\partial \delta b_{j}}{\partial a_{m}} .
$$

In (18.15) we have

$$
b_{j k, k}=\frac{\partial b_{j k}}{\partial a_{k}}=\frac{\partial b_{j k}}{\partial b_{r s}} b_{r, s k}=0,
$$

because $b_{r, s k}=\partial^{2} b_{r} / \partial a_{k} \partial a_{s}=\partial^{2} b_{r} / \partial a_{s} \partial a_{k}$ is symmetric in the indices $s$ and $k$, and the $(n-2)$-order subdeterminant of the determinant (18.9)

$$
\frac{\partial b_{j k}}{\partial b_{r, s}}=-\frac{\partial b_{j s}}{\partial b_{r, k}}
$$

is antisymmetric in these indices (see, for example, B. E. MAdELung [5]). Thus, (18.15) reduces to

$$
\frac{\partial \delta a_{j}}{\partial a_{j}}=b_{j m} \frac{\partial \delta b_{j}}{\partial a_{m}} .
$$


Analogous to equations (18.3) and (18.4) for the labeling coordinates $a_{j}$ and their variations $\delta a_{j}$ with respect to the coordinates $\left(x_{1}, x_{2}, \ldots, x_{n}, t\right)$ of the spacetime continuum, for the label coordinates $b_{j}$ and their variations $\delta b_{j}$ we have

$$
\begin{aligned}
b_{j} & =b_{j}\left(x_{1}, x_{2}, \ldots, x_{n}, t\right), \\
\delta b_{j} & =\frac{\partial b_{j}}{\partial x_{k}} \delta x_{k}+\frac{\partial b_{j}}{\partial t} \delta t .
\end{aligned}
$$

By elimination of the spatial coordinates $x_{1}, x_{2}, \ldots, x_{n}$ the coefficients $\partial b_{j} / \partial x_{k}$ and $\partial b_{j} / \partial t$ can be expressed as functions of $b_{1}, b_{2}, \ldots, b_{n}$ and $t$. Then, the right hand side of (18.18) can be transformed into

$$
b_{j m} \frac{\partial \delta b_{j}}{\partial a_{m}}=b_{j m} b_{k, m} \frac{\partial \delta b_{j}}{\partial b_{k}}
$$

so that from (18.18), with consideration of (18.12), we obtain

$$
\frac{\partial \delta a_{j}}{\partial a_{j}}=\frac{\partial \delta b_{j}}{\partial b_{j}}
$$

which proves the invariance of the form (18.10) with respect to the unimodular transformation (18.8) and (18.9) of the labeling coordinates.

\section{Corollary}

From (18.22) there follows the invariance relations

$$
\frac{\partial}{\partial a_{j}}\left(\frac{\partial a_{j}}{\partial x_{i}}\right)=\frac{\partial}{\partial b_{j}}\left(\frac{\partial b_{j}}{\partial x_{i}}\right) \quad(i=1,2, \ldots, n)
$$

and

$$
\frac{\partial}{\partial a_{j}}\left(\frac{\partial a_{j}}{\partial t}\right)=\frac{\partial}{\partial b_{j}}\left(\frac{\partial b_{j}}{\partial t}\right)
$$

with respect to unimodular transformation of the labeling coordinates, because the variations $\delta x_{1}, \delta x_{2}, \ldots, \delta x_{n}, \delta t$ are arbitrary and independent.

\section{Hydrodynamical interpretation}

The derived relations (18.10), or equivalently (18.22), (18.23) and (18.24) (especially, equation (18.24)) require additional explanation.

In (18.24) $\partial a_{j} / \partial t$ is the local time derivative of the labeling coordinate $a_{j}$ at the fixed point $\left\{x_{1}, x_{2}, \ldots, x_{n}\right\}$ of reference space, which arises due to the passage of labeled particles. If these changes are assigned to the particle labels that occur at the same time $t$ at neighboring spatial points, and if with these particle labels the expression on the left side of (18.24) is calculated, then the same result is invariably obtained in another system of labeling coordinates $b_{j}$ that is related to the system $a_{j}$ by a unimodular tranformation.

The interpretation of the formula (18.23) is similar to the interpretation of (18.10) and (18.22). The creation of these invariant forms is based on the hydrodynamical fact that all systems of labeling coordinates must contain the necessary number of eliminable coordinates (here $x_{1}, x_{2}, \ldots, x_{n}$ ).

The invariant form $F$ in equation (18.10) is in general a function of the coordinates of the space-time continuum, which depends on the physical behaviour of the fluid. If the labeling coordinates $a_{j}$ are selected to be the particle positions at time $t=0$, and the fluid is incompressible, then $F=0$, as can be seen from the continuity equation in Lagrangian form. Then, in equations (18.22), (18.23) and (18.24) the sums on either side vanish.

In the case of a compressible fluid, we obtain

$$
F=\frac{\partial \delta a_{j}}{\partial a_{j}}=\delta\left\{\ln \left(\frac{\rho}{\rho_{0}}\right)\right\}
$$

( $\rho=$ density, $\rho_{0}=$ initial density). This can be proved through logarithmic variation of the continuity equation

$$
\frac{\partial\left(a_{1}, a_{2}, \cdots, a_{n}\right)}{\partial\left(x_{1}, x_{2}, \cdots, x_{n}\right)}=\frac{\rho}{\rho_{0}}
$$

under the condition that the operators $\delta$ and $\partial / \partial x_{1}, \partial / \partial x_{2}, \ldots, \partial / \partial x_{n}$ are commutable.

\section{References}

[1] Bjerknes, V., Bjerknes, J., Solberg, H., u. T. BERGERON, Physikalische Hydrodynamik mit Anwendung auf die Dynamische Meteorologie, Berlin 1933, 51-53.

[2] Dryden, H. L., Murnaghan, F. D., and H. Bateman, Hydrodynamics, New York 1956, 32.

[3] ERTEl, H., Teorema sobre invariantes sustanciales de la Hidrodinámica. Gerl. Beitr. Geophys. 69 (1960), 290-293.

[4] Hollmann, G., Ein vollständiges System hydrodynamischer Erhaltungssätze. Arch. Meteorol. Geophys. Bioklim. A 14 (1964), 1-13.

[5] Madelung, E., Eie mathematischen Hilfsmittel des Physikers, 7. Aufl. Berlin/Göttingen/Heidelberg 1964, 145.

[6] NAAS, J., u. H. L. SCHMID, Mathematisches Wörterbuch, Bd. II, Berlin/Leipzig 1961, 787. 
[7] Reichardt, H., Vorlesungen über Vektor- und Tensorrechnung, Berlin 1957, 29.

[8] Schirokow, P. A., u. A. P. Schirokow, Affi ne Differentialgeometrie, Leipzig 1962, 142.
[9] Strubecker, K., Differentialgeometrie II, Berlin $1958,117-118$.

[10] Vidal Abascal, E., Introducción a la Geometría Diferencial, Madrid 1956, 277.

\section{ERTEL (1970a): A relationship between kinematical parameters of horizontal fbw fields in the atmosphere}

\section{Summary}

The quasilinear inertia terms in two different forms are compatible only if the determinant of the coeffi cients of the velocity components is equal to zero. This condition of compatibility gives a relationship between the parameters which characterize the kinematics of two-dimensional air movements. The simple example of the gradient wind discloses the meaning of this relationship.

"Observations show that every large-scale current in the atmosphere is nearly horizontal" (J. Holmboe, G. E. Forsythe, W. Gustin [5]). The research methods of the kinematics of horizontal fbw must develop integrating components of dynamic and synoptic meteorology (V. Bjerknes, Th. Hesselberg, O. Devik [1]; V. Bjerknes, J. Bjerknes, H. Solberg, T. Bergeron [2]; J. M. Jansa Guardiola [6]). That part of a horizontal fbw field that is in the immediate vicinity of any moving air (mass) parcel can be regarded with sufficient approximation as evenly varying. For such regions, a relation between various parameters of the fields of fbw will be derived here. For kinematic parameters this relation contains, on the one hand, the three components of the symmetrical deformation tensor and the spin of the air mass elements, and on the other hand, the streamline-tangential derivatives of the isotachs and isogons.

Let $x$ and $y$ be arbitrarily oriented orthogonal cartesian coordinates in the horizontal plane, which, together with an upward vertical $z$-coordinate, form a righthanded system. Based on this system, $v_{x}$ and $v_{y}$ are the horizontal components of the fbw velocity. We define $d v_{x} / d t$ and $d v_{y} / d t$ as the material derivatives, with $\partial v_{x} / \partial t$ and $\partial v_{y} / \partial t$ the local derivatives of the fbw components with respect to time, $t$, such that

$$
\frac{d v_{x}}{d t}-\frac{\partial v_{x}}{\partial t}=\frac{\partial v_{x}}{\partial x} v_{x}+\frac{\partial v_{x}}{\partial y} v_{y}
$$

and

$$
\frac{d v_{y}}{d t}-\frac{\partial v_{y}}{\partial t}=\frac{\partial v_{y}}{\partial x} v_{x}+\frac{\partial v_{y}}{\partial y} v_{y}
$$

are the nonlinear terms, those which generally contain the nonsymmetric gradient tensor (D. Morgenstern and I. Szabó [8])

$$
V=\left(\begin{array}{cc}
\frac{\partial v_{x}}{\partial x} & \frac{\partial v_{x}}{\partial y} \\
\frac{\partial v_{y}}{\partial x} & \frac{\partial v_{y}}{\partial y}
\end{array}\right)
$$

In addition, we use polar coordinates to represent the fbw field, with positive definite $v=\left(v_{x}^{2}+v_{y}^{2}\right)^{1 / 2}$ representing the fbw speed and $\alpha$ being the positive azimuthal angle, so that

$$
v_{x}=v \cos \alpha, \quad v_{y}=v \sin \alpha .
$$

The lines $v=$ const and $\alpha=$ const are the isotachs and isogons, respectively, of the fbw field and $\partial / \partial s=\cos \alpha(\partial / \partial x)+\sin \alpha(\partial / \partial y)$ is the streamlinetangential derivative, which with (19.1) and (19.2) implies

$$
\frac{d v_{x}}{d t}-\frac{\partial v_{x}}{\partial t}=v \frac{\partial v_{x}}{\partial s}, \quad \frac{d v_{y}}{d t}-\frac{\partial v_{y}}{\partial t}=v \frac{\partial v_{y}}{\partial s} .
$$

Because, in consideration of (19.4),

$$
\frac{\partial v_{x}}{\partial s}=\frac{\partial v}{\partial s} \cos \alpha-v \frac{\partial \alpha}{\partial s} \sin \alpha,
$$

and

$$
\frac{\partial v_{y}}{\partial s}=\frac{\partial v}{\partial s} \sin \alpha+v \frac{\partial \alpha}{\partial s} \cos \alpha,
$$

we can write expressions for the convection of $v_{x}$ and $v_{y}$ :

$$
\begin{aligned}
v \frac{\partial v_{x}}{\partial s} & =\frac{\partial v}{\partial s} v_{x}-v \frac{\partial \alpha}{\partial s} v_{y} \\
v \frac{\partial v_{y}}{\partial s} & =v \frac{\partial \alpha}{\partial s} v_{x}+\frac{\partial v}{\partial s} v_{y}
\end{aligned}
$$


which with (19.5) results in

$$
\begin{aligned}
& \frac{d v_{x}}{d t}-\frac{\partial v_{x}}{\partial t}=\frac{\partial v}{\partial s} v_{x}-v \frac{\partial \alpha}{\partial s} v_{y}, \\
& \frac{d v_{y}}{d t}-\frac{\partial v_{y}}{\partial t}=v \frac{\partial \alpha}{\partial s} v_{x}+\frac{\partial v}{\partial s} v_{y} .
\end{aligned}
$$

Subtraction of equation (19.10) from (19.1) and of equation (19.11) from (19.2) yields the homogeneous system, expressed in terms of both the fbw components $\left(v_{x}, v_{y}\right)$ :

$$
\begin{aligned}
& \left(\frac{\partial v_{x}}{\partial x}-\frac{\partial v}{\partial s}\right) v_{x}+\left(\frac{\partial v_{x}}{\partial y}+v \frac{\partial \alpha}{\partial s}\right) v_{y}=0, \\
& \left(\frac{\partial v_{y}}{\partial x}-v \frac{\partial \alpha}{\partial s}\right) v_{x}+\left(\frac{\partial v_{y}}{\partial y}-\frac{\partial v}{\partial s}\right) v_{y}=0,
\end{aligned}
$$

which, as evident from its derivation, must apply for nonstationary as well as stationary fbw fields.

We now go from the nonsymmetric gradient tensor to the symmetric deformation tensor (tensor of the deformation of the fbw)

$$
D=\left(\begin{array}{cc}
D_{x x} & D_{x y} \\
D_{y x} & D_{y y}
\end{array}\right)
$$

by defining the following components:

$$
\begin{aligned}
D_{x x} & =\frac{\partial v_{x}}{\partial x}, \\
D_{y y} & =\frac{\partial v_{y}}{\partial y}, \\
D_{x y}=D_{y x} & =\frac{1}{2}\left(\frac{\partial v_{x}}{\partial y}+\frac{\partial v_{y}}{\partial x}\right),
\end{aligned}
$$

and using the identities:

$$
\begin{aligned}
& \frac{\partial v_{x}}{\partial y}=D_{x y}-\frac{1}{2}\left(\frac{\partial v_{y}}{\partial x}-\frac{\partial v_{x}}{\partial y}\right)=D_{x y}-S, \\
& \frac{\partial v_{y}}{\partial x}=D_{x y}+\frac{1}{2}\left(\frac{\partial v_{y}}{\partial x}-\frac{\partial v_{x}}{\partial y}\right)=D_{x y}+S,
\end{aligned}
$$

where the spin (A. S. Ramsey [9]; O. G. Sutton [11])

$$
S=\frac{1}{2}\left(\frac{\partial v_{y}}{\partial x}-\frac{\partial v_{x}}{\partial y}\right)
$$

denotes the turning of an air (mass) parcel around the vertical $z$-axis.

The components $D_{x x}$ and $D_{y y}$ of the deformation tensor (19.14) describe the stretching and compression rates of the fluid parcels, while the component $D_{x y}$ expresses a shearing rate $(O$. Tietjens [12]). A necessary and sufficient condition for motion without deformation, analogous to a rigid body, is that all terms in the deformation tensor vanish (J. Serrin [10]).
The spin defined in (19.18) is the turning rate of air parcels relative to the $(x, y)$-system. Relative to the convection of the direction of fbw $\alpha$ the spin of a fluid particle is given by

$$
R=S-v \frac{\partial \alpha}{\partial s} .
$$

Now, through elimination of the components of the gradient tensor of the fbw in equations (19.12) and (19.13), and by means of (19.15), (19.16), and (19.17) the following system results:

$$
\begin{aligned}
& \left(D_{x x}-\frac{\partial v}{\partial s}\right) v_{x}+\left(D_{x y}-S+v \frac{\partial \alpha}{\partial s}\right) v_{y}=0 \\
& \left(D_{x y}+S-v \frac{\partial \alpha}{\partial s}\right) v_{x}+\left(D_{y y}-\frac{\partial v}{\partial s}\right) v_{y}=0
\end{aligned}
$$

or, considering (19.19), in a somewhat more abbreviated form:

$$
\begin{aligned}
& \left(D_{x x}-\frac{\partial v}{\partial s}\right) v_{x}+\left(D_{x y}-R\right) v_{y}=0, \\
& \left(D_{x y}+R\right) v_{x}+\left(D_{y y}-\frac{\partial v}{\partial s}\right) v_{y}=0 .
\end{aligned}
$$

The necessary and sufficient condition (M. Bocher [3]) for the compatability of equations (19.22) and (19.23) appears in determinant form as:

$$
\left|\begin{array}{cc}
D_{x x}-\frac{\partial v}{\partial s} & D_{x y}-R \\
D_{x y}+R & D_{y y}-\frac{\partial v}{\partial s}
\end{array}\right|=0
$$

that is, the equation

$$
\begin{aligned}
\left(\frac{\partial v}{\partial s}\right)^{2} & -\frac{\partial v}{\partial s}\left(D_{x x}+D_{y y}\right) \\
& +\left|\begin{array}{ll}
D_{x x} & D_{x y} \\
D_{y x} & D_{y y}
\end{array}\right|+R^{2}=0
\end{aligned}
$$

must be satisfied.

Equation (19.25) represents the sought-after relation between the kinematic parameters-their invariance in relation to rotations of the orthogonal $(x, y)$ coordinate system about the vertical $z$-axis is thereby assured, insomuch as it is well established that the trace $D_{x x}+D_{y y}$ and the determinant

$$
|D|=\left|\begin{array}{ll}
D_{x x} & D_{x y} \\
D_{x y} & D_{y y}
\end{array}\right|
$$


of the deformation tensor (19.14) are invariant (G. Joos and Th. Kaluza [7]; W. Weizel [13],[14]).

By means of the relation (19.25), it is simple to examine which horizontal fbws in the atmosphere lead to deformationless movement of individual air parcels. Since in this case $D_{x x}=D_{y y}=D_{x y}=0$ must apply, (19.25) reduces to the equation

$$
\left(\frac{\partial v}{\partial s}\right)^{2}+R^{2}=0,
$$

which involves the two conditions:

$$
\frac{\partial v}{\partial s}=0
$$

and

$$
R=S-v \frac{\partial \alpha}{\partial s}=0
$$

The first condition (19.28) is fulfilled by a stationary fbw gradient as a consequence of fbw parallel to the isobars (A. Defant and F. Defant [4]). Assuming that the air parcel motions are deformationless, the second condition (19.29) means that the spin $S$ must agree with the convection of the fbw direction; in the special case of geostrophic fbw ( $\partial \alpha / \partial s=0), S=0$.

Consider when the geostrophic fbw blows in the $x$ direction, with shear due to the change of the wind velocity $v_{x}$ in the gradient-parallel $y$-direction, thus the air parcels possess a spin $S$, its magnitude agreeing with the shear, whereby the signs of $S$ and $D_{x y}$ are opposite. In consideration of (19.29), the condition (19.25) now demands

$$
-D_{x y}^{2}+S^{2}=\left(S+D_{x y}\right)\left(S-D_{x y}\right)=0,
$$

which is satisfied since $D_{x y}=\frac{1}{2}\left(\partial v_{x} / \partial y\right)$ from (19.15) and $S=-\frac{1}{2}\left(\partial v_{x} / \partial y\right)$ from (19.18) and $S+D_{x y}=0$ identically. The spin is negative (anticyclonic) with positive shearing, and vice versa.

Consider the deformation of a horizontal square into a rhombus by the shearing effect $\frac{1}{2}\left(\partial v_{x} / \partial y\right)$. This operation turns the coordinate system of the original orthogonal diagonals in such a way that the orthogonality of the diagonals is conserved, according to a theorem of geometry. This turning is a simple example of the spin.

\section{References}

[1] Bjerknes, V., Th. Hesselberg, O. Devik: Dynamic Meteorology and Hydrography. Part II, Kinematics. Washington 1911.

[2] Bjerknes, V., J. Bjerknes, H. Solberg, T. Bergeron: Physikalische Hydrodynamik mit Anwendungen auf die dynamische Meteorologie. Berlin 1933.

[3] Bocher, M.: Introduction to Higher Algebra. New York 1964, p. 47.

[4] Defant, A., und F. Defant: Physikalische Dynamik der Atmosphäre. Frankfurt am Main, 1958, S. 121.

[5] Holmboe, J., G. E. Forsythe and W. Gustin: Dynamic Meteorology. Second Printing. New York (London) 1948, p. 173.

[6] Jansa Guardiola, J. M.: Tratado de Meteorología Teórica. Tomo II. Estática-Cinemática. Madrid 1960, pag. 152 .

[7] Joos, G., und Th. Kaluza: Höhere Mathematik für den Praktiker. Sechste verbesserte Auflage. Leipzig 1952, S. 118.

[8] Morgenstern, D., und I. Szabó: Vorlesungen über Theoretische Mechanik. Berlin (Göttingen) Heidelberg 1961, S. 79.

[9] Ramsey, A. S.: A Treatise on Hydromechanics. Part II. Hydrodynamics. Fourth Edition. Reprinted. London 1947, p. 27.

[10] Serrin, J.: Mathematical Principles of Classical Fluid Mechanics. Handbuch der Physik, Bd. III/1. Berlin (Göttingen) Heidelberg 1959, p. 139.

[11] Sutton, O. G.: Micrometeorology. A Study of Physical Processes in the Lowest Layers of the Earth's Atmosphere. London 1953, p. 23.

[12] Tietjens, O.: Hydro- und Aeromechanik nach Vorlesungen von L. Prandtl. Erster Band. Zweite Auflage. Wien 1944, S. 75.

[13] Weizel, W.: Physikalische Formelsammlung. Erster Band. Mannheim 1962, S. 48.

[14] Weizel, W.: Lehrbuch der Theoretischen Physik. Erster Band. Physik der Vorgänge. Berlin (Göttingen) Heidelberg 1963, S. 142. 


\section{ERTEL (1970b): Spin and deformation tensor in connection with isogons and isotachs in two-dimensional streaming fields}

\section{Summary}

In two-dimensional streams the acceleration can be expressed by isogons and by equivelocity contours, but it can be demonstrated also as a function of the two-dimensional deformation velocity and of the tensor of spin of fluid particles. On the basis of the condition of compatibility a connection of general validity can be obtained between all four mentioned quantities for both cases. That connection is quite independent both from the kind of fluid and from the characteristics in time of the streaming fi eld.

\section{Introduction}

In recent years, the field of hydrodynamics has adopted a remarkable development by introducing parameters from differential geometry (see, for example, H. Fortak [2], M. Olberg [3]). Continuing in this vein, the present work links elements of the kinematics on large scales (macro-kinematic parameters: Isotachs and Isogons) with elements of the kinematics on small scales (micro-kinematic parameters: Spin and components of the deformation rate of the individual fluid particles).

\section{Symbols}

From the compatibility condition (H. ERTEL [1])

$$
\left|\begin{array}{cc}
D_{x x}-\frac{\partial v}{\partial s} & D_{x y}-S+v \frac{\partial \alpha}{\partial s} \\
D_{x y}+S-v \frac{\partial \alpha}{\partial s} & D_{y y}-\frac{\partial v}{\partial s}
\end{array}\right|=0
$$

we get two forms of the convective acceleration of two-dimensional fbw fields, where, with an orthogonal cartesian coordinate system $(x, y)$, the following symbols are used:

$$
\begin{aligned}
D_{x x} & =\frac{\partial v_{x}}{\partial x} \\
D_{y y} & =\frac{\partial v_{y}}{\partial y} \\
D_{x y}=D_{y x} & =\frac{1}{2}\left(\frac{\partial v_{y}}{\partial x}+\frac{\partial v_{x}}{\partial y}\right),
\end{aligned}
$$

which are the components of the deformation rate tensor associated with the velocity components $\left(v_{x}, v_{y}\right)$. Furthermore, a fluid particle's spin (for example, O. G. SUTTON [5]) is

$$
S=\frac{1}{2}\left(\frac{\partial v_{y}}{\partial x}-\frac{\partial v_{x}}{\partial y}\right),
$$

the magnitude of the fbw velocity is

$$
v=\left(v_{x}^{2}+v_{y}^{2}\right)^{\frac{1}{2}}
$$

and the isogon angle, considered positive with counterclockwise rotation with respect to the $\mathrm{x}$-axis, is

$$
\alpha=\tan ^{-1}\left(\frac{v_{y}}{v_{x}}\right) .
$$

The curves $v=$ const are the isotachs and the curves $\alpha=$ const are the isogons (isoclines). The operator $\partial / \partial s$ in (20.1) is the derivative along the streamline. The derivative in the perpendicular direction, in the sense of counterclockwise rotation, is designated as $\partial / \partial n$, so one can write

$$
D_{x x}+D_{y y}=\frac{\partial v_{x}}{\partial x}+\frac{\partial v_{y}}{\partial y}=\frac{\partial v}{\partial s}+v \frac{\partial \alpha}{\partial n}
$$

and

$$
2 S=\frac{\partial v_{y}}{\partial x}-\frac{\partial v_{x}}{\partial y}=-\frac{\partial v}{\partial n}+v \frac{\partial \alpha}{\partial s},
$$

with the aid of

$$
v_{x}=v \cos \alpha, \quad v_{y}=v \sin \alpha
$$

and

$$
\begin{gathered}
\frac{\partial}{\partial s}=\cos \alpha \frac{\partial}{\partial x}+\sin \alpha \frac{\partial}{\partial y}, \\
\frac{\partial}{\partial n}=-\sin \alpha \frac{\partial}{\partial x}+\cos \alpha \frac{\partial}{\partial y}
\end{gathered}
$$

\section{A relation between macro- and micro-kinematical parameters in the flow field}

Instead of introducing three separate components of the deformation rate tensor, it is convenient to form a microkinematic parameter which is a combination of the components, namely the determinant

$$
D=\left|\begin{array}{ll}
D_{x x} & D_{x y} \\
D_{x y} & D_{y y}
\end{array}\right| .
$$

Such a quantity has the property of invariance under coordinate transformations (e.g., W. WEIZEL [6]). Then, it is a matter of developing a relationship between the two macrokinematic parameters $(v, \alpha)$ and the two microkinematic parameters $(S, D)$. 
The derivation of this relationship is then very simple. Expanding the compatibility determinant (20.1), the equation

$$
\begin{aligned}
\left(\frac{\partial v}{\partial s}\right)^{2}-\frac{\partial v}{\partial s} & \left(D_{x x}+D_{y y}\right) \\
& +D+\left(S-v \frac{\partial \alpha}{\partial s}\right)^{2}=0
\end{aligned}
$$

is obtained. First, (20.6) is substituted into (20.11):

$$
-v \frac{\partial \alpha}{\partial n} \frac{\partial v}{\partial s}+D+\left(S-v \frac{\partial \alpha}{\partial s}\right)^{2}=0
$$

Then, substituting the expression given for $2 S$ in (20.7) into

$$
\left(S-v \frac{\partial \alpha}{\partial s}\right)^{2}=S^{2}-2 S v \frac{\partial \alpha}{\partial s}+v^{2}\left(\frac{\partial \alpha}{\partial s}\right)^{2}
$$

yields

$$
\left(S-v \frac{\partial \alpha}{\partial s}\right)^{2}=S^{2}+v \frac{\partial \alpha}{\partial s} \frac{\partial v}{\partial n},
$$

which then transforms (20.12) into

$$
v\left(\frac{\partial \alpha}{\partial s} \frac{\partial v}{\partial n}-\frac{\partial \alpha}{\partial n} \frac{\partial v}{\partial s}\right)+D+S^{2}=0
$$

the sought-after equation that represents the relationship between $v, \alpha, D$, and $S$. Using the orthogonal cartesian coordinates $(x, y)$ instead of the natural coordinates $(s, n)$, one can also write

$$
v\left(\frac{\partial \alpha}{\partial x} \frac{\partial v}{\partial y}-\frac{\partial \alpha}{\partial y} \frac{\partial v}{\partial x}\right)+D+S^{2}=0
$$

since the terms in parentheses in (20.15) and (20.16) are invariant under rotations of the two-dimensional coordinate system about the normal z-direction $(x, y, z=\mathrm{co}-$ ordinate system). Applying the symbols of vector analysis, the equations $(20.15,20.16)$ take on the form

$$
v(\nabla \alpha \times \nabla v)_{z}+D+S^{2}=0,
$$

where the cross product represents vector multiplication of the horizontal gradients of $\alpha$ and $v$.

As a sample calculation, we consider a circular vortex centered at an origin $r=0$ with constant angular velocity $\omega$, like that for rigid body rotation. In this case, all deformation components vanish (e.g., J. SERRIN [4]), thus $D$ dissappears. Here $v=r \omega$,

$$
\frac{\partial}{\partial s}=\frac{\partial}{r \partial \alpha}, \quad \frac{\partial}{\partial n}=-\frac{\partial}{\partial r},
$$

and equation (20.15) immediately results in:

$$
-\omega^{2}+S^{2}=0
$$

that is, the spin of the individual fluid particles corresponds to the angular velocity of the entire circular vortex.

It is still noted, that the equations (20.15), (20.16) and (20.17) apply to arbitrary flids, as well as to stationary and nonstationary two dimensional fbws, according to the purely kinetic nature of the generally accepted compatibility determinant.

\section{References}

1. ERTEL, H.: Eine Relation zwischen kinematischen Parametern horizontaler Strömungsfelder in der Atmosphäre. Idójárás, Budapest, 74 (1970), 98.

2. Fortak, H.: Acta Hydrophysica, Berlin XIV (1969), 57.

3. Olberg, M.: Acta Hydrophysica, Berlin XIV (1969), 181.

4. SERrin, J.: Mathematical Principles of Classical Fluid Mechanics. Handbuch der Physik, Bd. VIII/1. Berlin/Göttingen/Heidelberg 1959, 139.

5. Sutton, O. G.: Micrometeorology. London 1953, 23.

6. WeIzel, W.: Physikalische Formelsammlung. Erster Band. Mannheim 1962, 48.

\section{ERTEL (1970c): Transformation of the differential form of the Weber hydro- dynamic equations in relation to the Earth's rotation}

\section{Summary}

We derive a system of equations that originate from the differential form of the Weber formulas of hydrodynamics, including the effects of the Earth's rotation. With this exten- sion the Weber formulas are of importance for meteorology and hydrography. The direct relationship of the extended Weber formulas with the circulation theorem of V. Bjerknes is easily deduced, as is the variational principle of atmospheric 
dynamics of H. Ertel.

\section{Symbols}

In the notation of vector and tensor analysis, including the summation convention, the hydrodynamic momentum equations for autobarotropic fbws, relative to an orthogonal cartesian coordinate system $x_{j}(j=$ $1,2,3)$ fixed with respect to the rotating Earth, can be written

$$
\frac{d v_{j}}{d t}-2 \omega_{j k} v_{k}=-\frac{\partial W}{\partial x_{j}}
$$

where $d / d t=$ individual (material) time derivative, $v_{j}=d x_{j} / d t=$ velocity components, $\omega_{j k}=-\omega_{k j}=$ Coriolis parameters $(j, k=1,2,3)$ for the Earth's rotation, $W=$ potential energy $=V+\int \alpha d p, V=$ potential for the force of gravity, and $\int \alpha d p=$ autobarotropic function of the specific volume $\alpha=\alpha(p)$. The origin $x_{j}=0$ of the coordinate system is any point on the Earth's axis.

\section{Differential form of the Weber equations}

The Weber equations of hydrodynamics use the time-independent Lagrangian coordinates $a_{i}(i=$ $1,2,3)$ as labels of individual particles; they are obtained from the Euler equations (21.1) through multiplication by $\partial x_{j} / \partial a_{i}$ and summation over the index $j$, resulting in the Lagrangian system

$$
\frac{d v_{j}}{d t} \frac{\partial x_{j}}{\partial a_{i}}-2 \omega_{j k} v_{k} \frac{\partial x_{j}}{\partial a_{i}}=-\frac{\partial W}{\partial a_{i}} .
$$

Because

$$
\frac{d v_{j}}{d t} \frac{\partial x_{j}}{\partial a_{i}}=\frac{d}{d t}\left(v_{j} \frac{\partial x_{j}}{\partial a_{i}}\right)-\frac{\partial}{\partial a_{i}}\left(\frac{v^{2}}{2}\right),
$$

where $v^{2}=v_{j} v_{j}$, we can write (21.1) as

$$
\frac{d}{d t}\left(v_{j} \frac{\partial x_{j}}{\partial a_{i}}\right)-2 \omega_{j k} v_{k} \frac{\partial x_{j}}{\partial a_{i}}=\frac{\partial \Omega}{\partial a_{i}},
$$

where $\Omega$ is the difference between kinetic and potential energy:

$$
\Omega=\frac{1}{2} v^{2}-W,
$$

which is the (Helmholtz) kinetic potential.

In the absense of rotation $\left(\omega_{j k}=0\right), V$ (in $W$ and/or $\Omega$ ) assumes the simple meaning of the gravitational potential. By time integration of the formulas (21.4), together with $\omega_{j k}=0$, the Weber formulas would result (Ref.: [3]-[10]). Thus, the formulas (21.4) can be regarded as the differential form of the Weber formulas with the inclusion of the Earth's rotation.

\section{Transformation of the differential form of the We- ber formulas with inclusion of the Coriolis terms}

We now try to transform the formulas (21.4) in such a manner that the Coriolis terms, $-2 \omega_{j k} v_{k}\left(\partial x_{j} / \partial a_{i}\right)=$ $-2 \omega_{j k}\left(d x_{k} / d t\right)\left(\partial x_{j} / \partial a_{i}\right)$, can be split into two parts involving the operators $d / d t$ and $\partial / \partial a_{i}$. For this purpose the following identity is useful:

$$
\begin{aligned}
2 \omega_{j k} v_{k} \frac{\partial x_{j}}{\partial a_{i}} & =\frac{\partial}{\partial a_{i}}\left(\omega_{j k} x_{j} \frac{d x_{k}}{d t}\right) \\
& -\frac{d}{d t}\left(\omega_{j k} x_{j} \frac{\partial x_{k}}{\partial a_{i}}\right) .
\end{aligned}
$$

The correctness of this identity can be easily verified by evaluation of the derivatives with consideration of the commutative property of the operators $d / d t$ and $\partial / \partial a_{i}$. The first term of the right side of (21.6) can be expanded as

$$
\begin{aligned}
\frac{\partial}{\partial a_{i}}\left(\omega_{j k} x_{j} \frac{d x_{k}}{d t}\right) & =\omega_{j k} \frac{\partial x_{j}}{\partial a_{i}} \frac{d x_{k}}{d t} \\
& +\omega_{j k} x_{j} \frac{d}{d t} \frac{\partial x_{k}}{\partial a_{i}},
\end{aligned}
$$

and the second term as

$$
\begin{aligned}
-\frac{d}{d t}\left(\omega_{j k} x_{j} \frac{\partial x_{k}}{\partial a_{i}}\right) & =-\omega_{j k} \frac{d x_{j}}{d t} \frac{\partial x_{k}}{\partial a_{i}} \\
& -\omega_{j k} x_{j} \frac{d}{d t} \frac{\partial x_{k}}{\partial a_{i}} .
\end{aligned}
$$

The addition of (21.7) and (21.8), with consideration of

$$
\begin{aligned}
\omega_{j k} \frac{\partial x_{j}}{\partial a_{i}} \frac{d x_{k}}{d t} & -\omega_{j k} \frac{d x_{j}}{d t} \frac{\partial x_{k}}{\partial a_{i}} \\
& =\omega_{j k} v_{k} \frac{\partial x_{j}}{\partial a_{i}}-\omega_{k j} v_{k} \frac{\partial x_{j}}{\partial a_{i}}
\end{aligned}
$$

and the antisymmetry of the Coriolis components $\omega_{k j}$, verifies the identity (21.6).

The substitution of the identity (21.6) in (21.4) transforms the differential form of the Weber formulas with the Coriolis terms into the system

$$
\begin{aligned}
\frac{d}{d t}\left(v_{j} \frac{\partial x_{j}}{\partial a_{i}}\right. & \left.+\omega_{j k} x_{j} \frac{\partial x_{k}}{\partial a_{i}}\right) \\
& =\frac{\partial}{\partial a_{i}}\left(\Omega+\omega_{j k} x_{j} \frac{d x_{k}}{d t}\right)
\end{aligned}
$$

which is very suitable for applications to meteorology and hydrography, as the following examples show.

\section{Applications}


The multiplication of the formulas (21.10) with the differentials $d a_{i}$ of the time-independent Lagrangian labeling coordinates $a_{i}$ and summation over the index $i$ results in

$$
\begin{aligned}
d a_{i} \frac{d}{d t}\left(v_{j} \frac{\partial x_{j}}{\partial a_{i}}\right. & \left.+\omega_{j k} x_{j} \frac{\partial x_{k}}{\partial a_{i}}\right) \\
& =d\left(\Omega+\omega_{j k} x_{j} \frac{d x_{k}}{d t}\right),
\end{aligned}
$$

from which, by integration over a closed material curve $C$, it follows that

$$
\frac{d}{d t} \int_{C}\left(v_{j} d x_{j}+\omega_{j k} x_{j} d x_{k}\right)=0
$$

This is the conservation theorem for the absolute circulation in autobarotropic currents (Ref.: [1]), where $\int_{C} v_{j} d x_{j}$ is the circulation relative to the Earth and the second integral is twice the absolute value of the Earth's rotation vector $(\omega)$ multiplied by the projection $F$ of the closed curve $C$ on the equatorial plane (or on a plane parallel to the equatorial plane). In other words,

$$
\begin{aligned}
\int_{C} \omega_{j k} x_{j} d x_{k} & =\int_{C} \omega_{j k} \frac{1}{2}\left(x_{j} d x_{k}-x_{k} d x_{j}\right) \\
& =2 \omega F
\end{aligned}
$$

where $\omega_{1}=\omega_{23}=-\omega_{32}, \omega_{2}=\omega_{31}=-\omega_{13}$, and $\omega_{3}=$ $\omega_{12}=-\omega_{21}$ are the three components $\left(\omega_{1}, \omega_{2}, \omega_{3}\right)$ of the Earth's rotation vector.

In just as simple a fashion one can obtain from (21.10) the variational principle of atmospheric dynamics (Ref.: [2]). Multiplying (21.10) by the variations $\delta a_{i}$ of the time-independent Lagrangian labeling coordinates and then summing over the index $i$, we obtain the formulas

$$
\begin{aligned}
\frac{d}{d t}\left(v_{j} \delta x_{j}+\omega_{j k} x_{j} \delta x_{k}\right) & =\delta a_{i} \frac{\partial}{\partial a_{i}}\left(\Omega+\omega_{j k} x_{j} \frac{d x_{k}}{d t}\right) \\
& =\delta\left(\Omega+\omega_{j k} x_{j} v_{k}\right) .
\end{aligned}
$$

Integrating over the time interval $t_{2}-t_{1}$, and noting that the variations $\delta x_{j}=\left(\partial x_{j} / \partial a_{i}\right) \delta a_{i}$ vanish at the end points $t_{1}$ and $t_{2}$ for all indices $j$, the variational principle of atmospheric dynamics in the form

$$
0=\delta \int_{t_{1}}^{t_{2}} L d t
$$

results where the (generalized) Lagrange function

$$
L=\Omega+\omega_{j k} x_{j} v_{k},
$$

contains the kinetic potential $\Omega$ and the parallel component of the spin of a particle around the coordinate origin (which is on the Earth's axis), since the term $\omega_{j k} x_{j} v_{k}$ represents simply the dot product of the Earth's rotation vector with the spin vector.

\section{References}

[1] Bjerknes, V., Bjerknes, J., Solberg, H., u. T. BERGERON, Physikalische Hydrodynamik mit Anwendung auf die dynamische Meteorologie, Berlin 1933, 147.

[2] Ertel, H., Methoden und Probleme der dynamischen Meteorologie, Berlin 1938, 41; amerikanische Ausgabe: Ann Arbor (Mich.) 1943.

[3] LAMB, H., Hydrodynamics, 6th (rev.) ed., $1^{\text {st }}$ amer. ed. New York 1945, 14.

[4] Lichtenstein, L., Grundlagen der Hydromechanik, Berlin 1929, 408.

[5] Long, R. R., Mechanics of Solids and Fluids, Englewood Cliffs (N.J.) 1961, 129.

[6] Oswatitsch, K., Physikalische Grundlagen der Strömungslehre, in: Handbuch der Physik, Bd. VIII/1, Berlin/Göttingen/Heidelberg 1959, 32.

[7] Schaefer, C., Einführung in die theoretische Physik, 1. Bd., 4. (durchges.) Aufl. Berlin 1944, 794.

[8] Serrin, J., Mathematical Principles of Classical Fluid Mechanics, in: Encyclopedia of Physics, Vol. VIII/1, Berlin/Göttingen/Heidelberg 1959, 169.

[9] Weber, H., Die partiellen Differentialgleichungen der mathematischen Physik, nach Riemann's Vorlesungen bearbeitet, 2. Bd., 6. Aufl. Braunschweig 1919, 419.

[10] Weizel, W., Physikalische Formelsammlung. 1. Bd., Mannheim 1962, 89. 